Portland State University

PDXScholar

Fall 12-1-2014

\title{
Selective Indicators for Optical Determination of Disease Biomarkers
}

Lovemore Hakuna

Portland State University

Follow this and additional works at: https://pdxscholar.library.pdx.edu/open_access_etds

Part of the Diagnosis Commons, and the Other Chemistry Commons Let us know how access to this document benefits you.

\section{Recommended Citation}

Hakuna, Lovemore, "Selective Indicators for Optical Determination of Disease Biomarkers" (2014). Dissertations and Theses. Paper 2053.

https://doi.org/10.15760/etd.2052

This Dissertation is brought to you for free and open access. It has been accepted for inclusion in Dissertations and Theses by an authorized administrator of PDXScholar. Please contact us if we can make this document more accessible: pdxscholar@pdx.edu. 
Selective Indicators for Optical Determination of Disease Biomarkers

by

Lovemore Hakuna

A dissertation submitted in partial fulfillment of the requirements for the degree of

Doctor of Philosophy

in

Chemistry

Dissertation Committee:

Robert M. Strongin, Chair

Carl C. Wamser

Reuben H. Simoyi

Jonathan Abramson

Portland State University

2014 


\begin{abstract}
The most abundant biological thiols, homocysteine (Hcy), cysteine (Cys) and glutathione (GSH) have been the subject of intense research due to their association with a wide range of diseases. They play a key role in maintaining the redox status of biological systems. Selective detection methods for these thiols are challenging due to their similar structures and properties. Current commercially available detection methods use separations, fragile and expensive enzymatic or immunogenic materials and complex instrumentation. This has led to a global effort towards developing simple and inexpensive optical probes and indicators selective for specific biological thiols.

Highly selective chemical probes and simple methods for detection and potential quantification of Hcy and GSH in their natural biological media have been developed. These indicators and methods are relatively simple and inexpensive for potential application at point of care. The selective detection of Hcy using novel asymmetric viologen chemical probes at room temperature is described as well as the use of commercially available materials under photochemical conditions. These probes respond linearly proportional to increasing Hcy concentrations, potentially enabling the monitoring of Hcy levels in human plasma. Additionally, new methods for the selective determination of GSH in human plasma, as well as its quantitation in whole blood deposited on filter paper (dried blood spots), is also presented herein.
\end{abstract}




\section{DEDICATION}

In the fullness of time God makes all things possible. Thank you Lord, thus far you have taken me!

To my beloved mother, it has been 8 years since you departed but it feels like yesterday, I love you and thanks for all your sacrifices. 


\section{ACKNOWLEDGEMENTS}

Firstly, I would like to express my sincere gratitude to my research advisor,

Dr Robert M. Strongin for his invaluable support, professional guidance and affording me the opportunity to work in his group.

My special thanks are extended to my research committee members, Dr. Carl C. Wamser, Dr. Reuben H. Simoyi and Dr Jonathan Abramson for their time and support in my studies.

To Dr. Jorge Escobedo, Dr. Martha Sibrian-Vazquez and Dr Mark Lowry, thanks for all your expertise and massive knowledge you shared with me during the course of my studies. I wish to acknowledge my former and current colleagues, Brandon Doughan for assisting with method optimization, Dr Soojin Lim, Aabha Barve, Yixing Guo, Davin Rautiola, Thomas Seidl, Jialu Wang, Shelly Chu, Lei Wang for their support. I'm also very grateful for the assistance provided by Dr. Wilbes Mbiya with EPR studies and Nicholas Day with cyclic voltammetry work.

Finally, I would like to express my deepest gratitude to my family for their prayers, moral support and encouragement throughout my studies especially the love of my life, Nolline and my beloved son, Sean Tinotenda. Thank you for your love and sacrifices to make this dream come true. 


\section{TABLE OF CONTENTS}

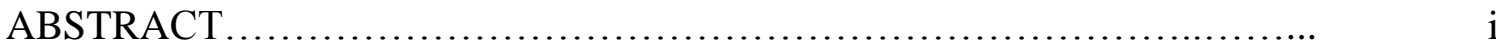







LIST OF FIGURES....................................................... viii

LIST OF ABBREVIATIONS ............................................. xii

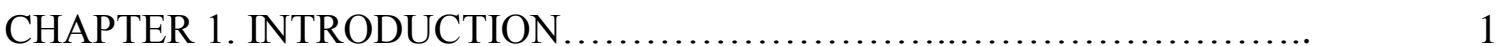

1.1 Background and significance of homocysteine......................... 1

1.1.1 Structures and composition of biological ........................

1.1.2 Significance of Hcy to public .................................. 2

1.2 Hcy controversy................................................ 6

CHAPTER 2. HOMOCYSTEINE DETECTION METHODS .................. 10

2.1 Current clinical methods........................................... $\quad 10$

2.2 Optical methods.................................................... 11

2.2.1 Hcy/Cys selective probes over GSH.......................... 11

2.2.2 Hcy selective probes...................................... 13

2.2.3 Hcy selective probes based on free radical chemistry ................ 16

CHAPTER 3. PHOTOCHEMICAL METHOD FOR SELECTIVE DETECTION OF TOTAL HCY IN PLASMA................................ 23

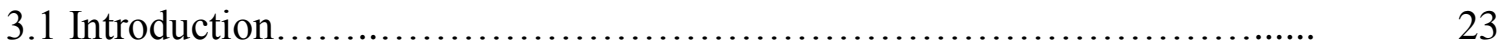


3.2 Results and discussion.............................................. 24

3.3 Experimental section............................................... 31

3.3.1 Materials and instruments...................................... 31

3.3.2 Reduction of human blood plasma............................... $\quad 31$

3.3.3 General procedure for the photolysis of thiols in the presence of viologens.......................................................... 31

3.4 Conclusion........................................................... $\quad 32$

CHAPTER 4. DETECTION ON OF HCY USING NOVEL ASYMMETRIC VIOLOGEN PROBES ................................................. 33

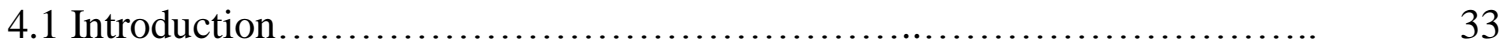

4.2 Results and discussion..........................................

4.2.1 Cyclic voltammetry studies................................... 35

4.2.2 Reactivity with aminothiols........................................ $\quad 37$

4.2.3 Mechanistic studies........................................... 46

4.3 Experimental Section.............................................. 48

4.3.1 Materials and instruments...................................... 48

4.3.2 Synthesis of asymmetric viologen probes.......................... $\quad 49$

4.3.3 Cyclic voltammetry ........................................... 57

4.3.4 General procedure for the UV/Vis absorption responses of asymmetric viologen probes towards thiols.............................. 57

4.4 Conclusion........................................................... 58

CHAPTER 5. SELECTIVE DETECTION OF GSH USING OPTICAL

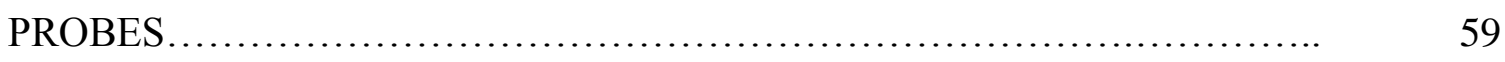

5.1 Significance of GSH to public health................................. 59

5.2 Current methods for GSH detection ................................. 60 


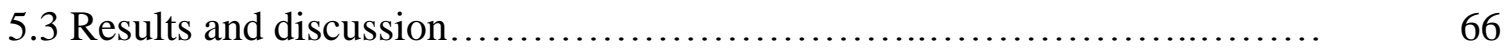

5.3.1 Acrylate-based probes.......................................... 66

5.3.2 Improved resorufin-acrylate selectivity towards GSH................ 69

5.3.3 Asymmetric viologen-based probe .............................. $\quad 73$





5.4.2 Synthesis of NIR acrylate probe................................... $\quad 78$

5.4.3 CTAB-mediated spectral responses of acrylate probes towards thiols general procedure ................................................. 80

5.5 Conclusion ....................................................... 80

CHAPTER 6. TOWARDS DEVELOPING A METHOD FOR DETECTION AND QUANTITATION OF GSH IN WHOLE BLOOD DEPOSITED ON FILTER PAPER ........................................................ 81

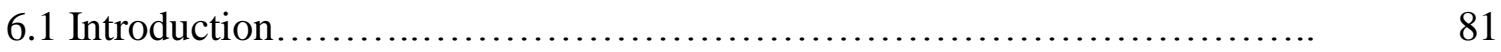

6.2 Results and discussion............................................ 83

6.3 Experimental section............................................... 90

6.3.1 Fractionation of GSH.......................................... 90

6.3.2 CTAB-mediated spectral responses of acrylate probes to GSH fractions ......................................................... 91

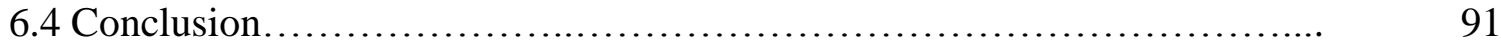

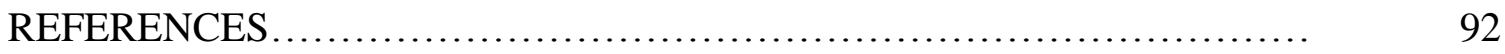

APPENDIX A. SUPPORTING INFORMATION ........................... 112

APPENDIX B. CHARACTERIZATION OF COMPOUNDS .................. 117 


\section{LIST OF TABLES}

Table 4.1 Reduction potentials of symmetric viologens and Hcy pH endpoint...

Table 4.2 Reduction potentials of novel asymmetric viologens vs methyl

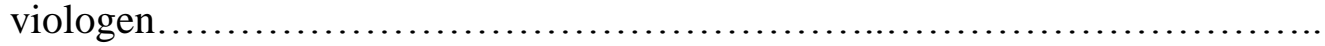

Table 5.1 Determination of GSH content in 10\% deproteinized human plasma






\section{LIST OF FIGURES}

Figure 1.1 Structures of selected biological thiols.......................... 2

Figure 1.2 Pathways for the metabolism of Hcy......................... 3

Figure 2.1 Substitution reaction of NBD-Cl (4) with aminothiols.............. 12

Figure 2.2 Reaction of 7 with Hcy/Cys.................................

Figure 2.3 Possible recognition mechanism of 9 with Hcy................... 13

Figure 2.4 Proposed mechanism for Hcy selectivity by the mesoporous silica



Figure 2.5 Sensing reaction of $\mathbf{1 3}$ with Hcy............................. 15

Figure 2.6 Mechanism of signal transduction in generic aldehyde-bearing

Figure 2.7 Kinetically favored HAT reaction for Hcy

Figure 2.8 Selective detection of Hcy in human plasma spiked with $4.0 \mathrm{mM}$ thiols.

Figure 2.9 Reaction mechanism for selective detection of Hcy using $\mathrm{MV}^{2+}$ at neutral $\mathrm{pH}$

Figure 2.10 Improved thermal method for detection of Hcy.....

Figure 2.11 Visual detection of Hcy in human serum calibration standards......

Figure 3.1 Response of $\mathrm{MV}^{2+}$ towards various thiols upon exposure to direct

Figure 3.2 Spectral response of $\mathrm{BV}^{2+}$ towards various spiked thiols in human blood plasma upon irradiation........................................... 26

Figure 3.3 Mechanism for the detection of Hcy by photolysis.................. 27

Figure 3.4 Spectral response of $\mathrm{BV}^{2+}$ towards various spiked thiols in reduced human plasma upon irradiation...........................................

Figure 3.5 Spectral response of $\mathrm{BV}^{2+}$ towards increasing levels of spiked Hcy

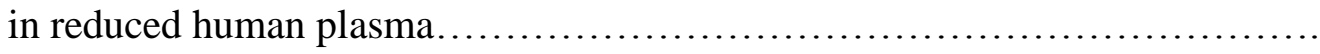


Figure 3.6 Spectral response of $\mathrm{BV}^{2+}$ towards various spiked amino acids in human plasma........................................................ 30

Figure 3.7 Summary of the photochemical method for detection of tHcy........ 32

Figure 4.1 Structures of novel asymmetric viologen probes................ 35

Figure 4.2 Response of $\mathrm{ACCV}^{2+}(27)$ towards various amino thiols at $\mathrm{pH} 8 \ldots \ldots . \quad 37$

Figure 4.3 Spectral response of $\mathrm{NCV}^{2+}(\mathbf{2 5})$ towards various amino thiols at $\mathrm{pH} 8$.......

Figure 4.4 Time-dependent spectral response of $\mathrm{ACNV}^{2+}(\mathbf{2 6})$ towards various amino thiols.

Figure 4.5 Optical sensing behavior of $\mathrm{ACNV}^{2+}(\mathbf{2 6})$ towards Hcy and Cys at $\mathrm{pH} 8$

Figure 4.6 Time-dependent spectral response of $\mathrm{PhNV}^{2+}(\mathbf{2 3})$ towards various amino thiols at $\mathrm{pH} 8$

Figure 4.7 Spectral response of $2.5 \mathrm{mM} \mathrm{PhNV}^{2+}(\mathbf{2 3})$ towards various amino



Figure 4.8 Spectral response of $2 \mathrm{mM} \mathrm{PhNV}^{2+}(\mathbf{2 3})$ towards various amino



Figure 4.9 Time-dependent spectral response of $\mathrm{PhNV}^{2+}(\mathbf{2 3})$ towards various

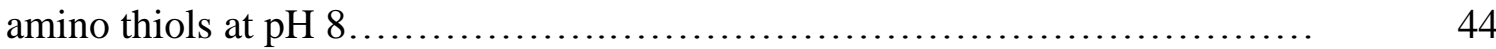

Figure 4.10 Concentration dependent responses of $\mathbf{2 3}(2 \mathrm{mM})$ towards Hcy....

Figure 4.11 Spectral response of $\mathrm{PhNV}^{2+}(\mathbf{2 3})$ towards various spiked thiols in deproteinized human plasma.

Figure 4.12 EPR spectra of $\mathrm{PhNV}^{2+}(\mathbf{2 3}),(20 \mathrm{mM})$ in the presence and absence



Figure 4.13 Response of $\mathrm{PhNV}^{2+}(\mathbf{2 3})$ towards Hcy, MPA and BME........... 48

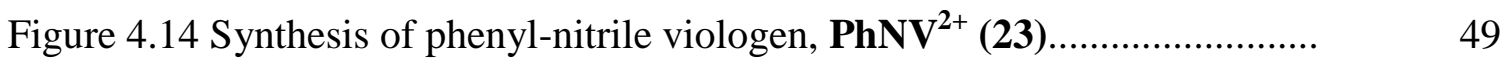

Figure 4.15 Synthesis of phenyl-acetyl coumarin viologen, $\mathbf{P h C V}^{\mathbf{2 +}}(\mathbf{2 4 )} \ldots \ldots \ldots$

Figure 4.16 Synthesis of Nitrile-acetyl coumarin viologen, $\mathbf{N C V}^{2+}(\mathbf{2 5}) \ldots \ldots \ldots .$.

Figure 4.17 Synthesis of Acetyl-nitrile viologen, $\mathbf{A C N V}^{\mathbf{2 +}}(\mathbf{2 6}) \ldots \ldots \ldots \ldots \ldots \ldots \ldots$ 
Figure 4.18 Synthesis of Acetyl-coumarin viologen, $\mathbf{A C C V}^{2+}(\mathbf{2 7}) \ldots \ldots \ldots \ldots \ldots . . . \ldots$

Figure 5.1 Michael addition reaction of 41 with thiols..................... 61

Figure 5.2 Proposed mechanism for discrimination of Cys from Hcy using

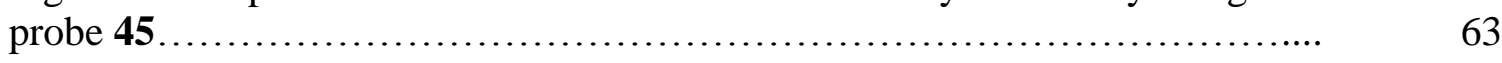

Figure 5.3 Intramolecular Williamson ether reaction....................... 64

Figure 5.4 Proposed Model for Selenium-Mediated Micellar.................. $\quad 65$

Figure 5.5 Structures of acrylate-based probes........................... 66

Figure 5.6 CTAB-mediated response of $\mathbf{4 9}$ towards various amino thiols at



Figure 5.7 Surfactant-mediated responses of solutions containing 50-CTAB

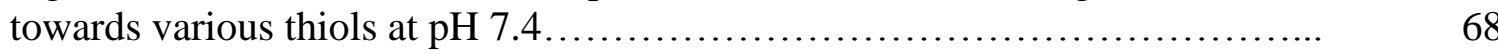

Figure 5.8 Proposed mechanism for the selective detection of GSH in CTAB



Figure 5.9 Spectral behavior of the probe-CTAB system under different $\mathrm{pH}$ conditions.............................................................

Figure 5.10 Surfactant mediated response of the probe-CTAB system towards

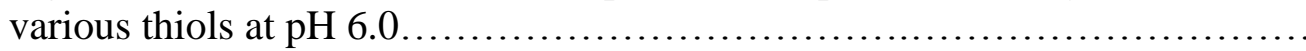

Figure 5.11 Absorption spectra of $\mathbf{5 0}(2.5 \mu \mathrm{M})$ upon addition of thiols at $\mathrm{pH} 6$

Figure 5.12 Determination of GSH in human plasma using the probe-CTAB system.

Figure 5.13 Spectral response of $\mathrm{PhCV}^{2+}(\mathbf{2 4})$ towards various amino thiols at $\mathrm{pH} 9$.

Figure 5.14 Absorption spectral changes of $\mathbf{2 4}$ upon addition of increasing concentrations of GSH...................................................

Figure 5.15 EPR spectrum of $\mathrm{PhCV}^{2+}(\mathbf{2 4}),(20 \mathrm{mM})$ in the presence and absence of GSH.

Figure 5.16 Energy minimized structures of $\mathbf{2 4}$ (left) and $\mathbf{2 4}$ in the presence of GSH (right) 
Figure 6.1 A method for detection of GSH in whole blood deposited on a filter



Figure 6.2 Absorption responses of DBS fractions......................... 84

Figure 6.3 Spectral response of $\mathbf{5 0}$ towards various DBS fractions.............. 85

Figure 6.4 Absorption responses of HSA fractions........................... 86

Figure 6.5 Absorption responses of $\mathrm{Hb}$ fractions............................. 86

Figure 6.6 Spectral response of $\mathbf{5 0}$ towards various $\mathrm{Hb}$ fractions................ 87

Figure 6.7 Spectral response of $\mathbf{5 0}$ towards DBS fractions at $3 \mathrm{~h}$ vs $24 \mathrm{~h} \ldots \ldots \ldots . . \quad 88$

Figure 6.8 Spectral response of $\mathbf{5 0}$ towards DBS spiked with various

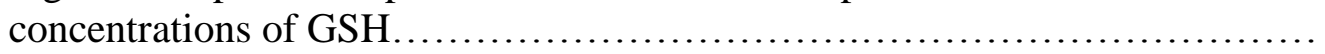

Figure 6.9 Comparison of commercial GSH fractions vs non-fractionated GSH............................................................. 


\section{LIST OF ABBREVIATIONS}

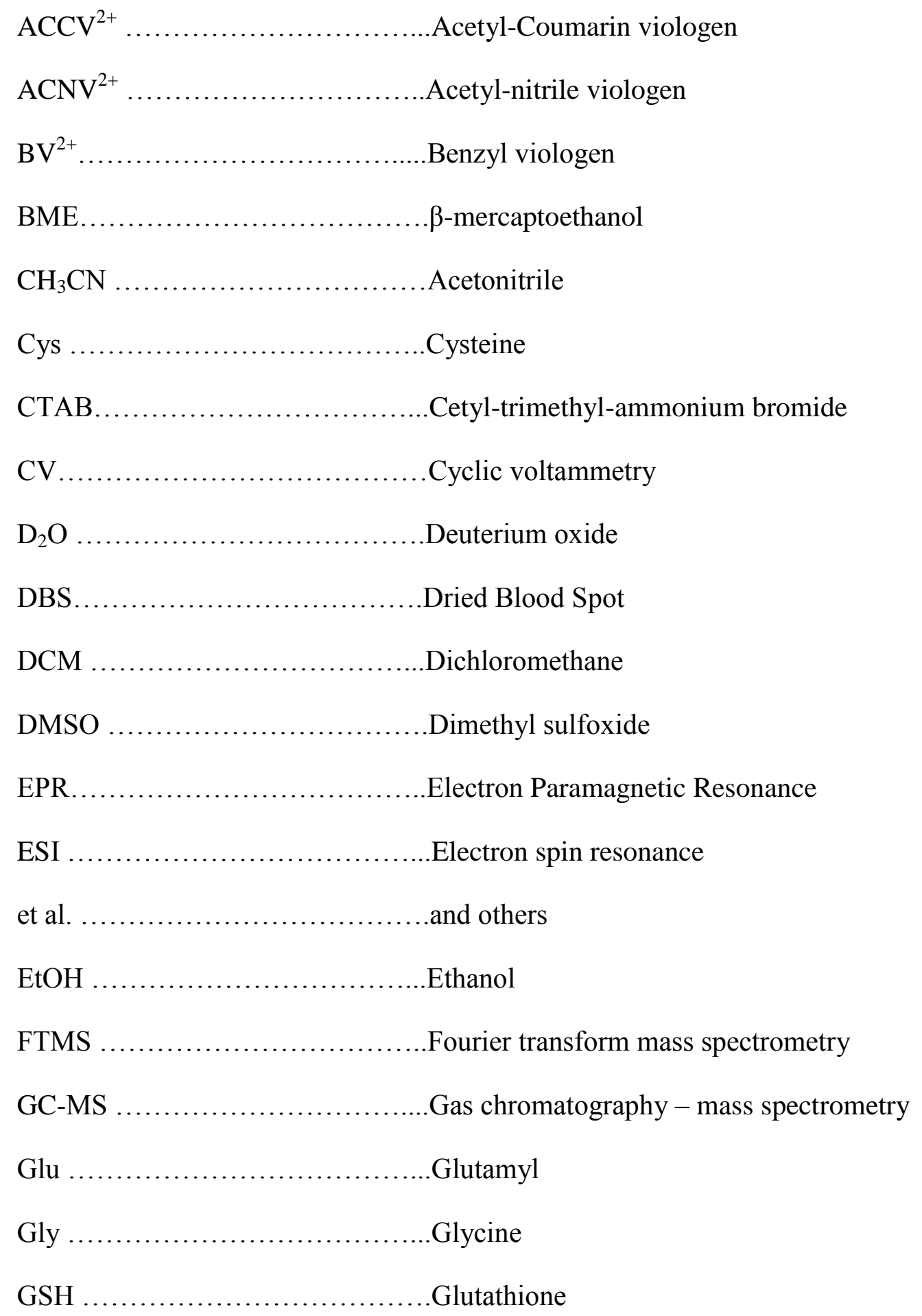




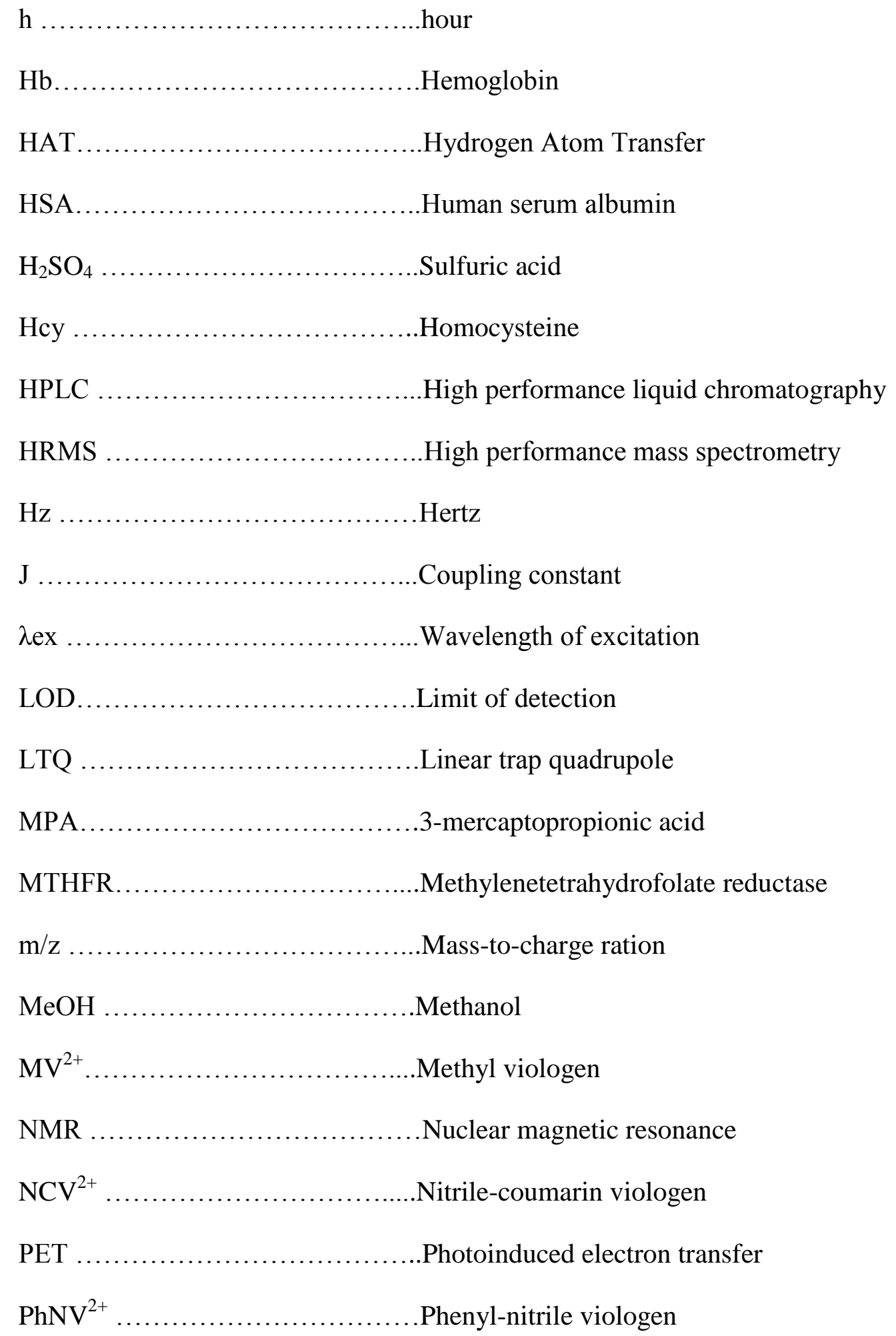




\begin{tabular}{|c|c|}
\hline $\mathrm{PhCV}^{2+}$. & ...Phenyl-coumarin viologen \\
\hline $\mathrm{pKa} \ldots$ & ...Logarithmic acidity constant \\
\hline ppm & ...Parts per million \\
\hline $\mathrm{rt} \ldots \ldots$ & ...Room temperature \\
\hline ROS..... & ..Reactive oxygen species \\
\hline TCEP... & ..Tris(2-carboxyethyl)phosphine \\
\hline SAH . & ..S-adenosyl homocysteine \\
\hline SAM . & ..S-adenosyl methionine \\
\hline
\end{tabular}




\section{CHAPTER 1: INTRODUCTION}

\subsection{Background and significance of homocysteine (Hcy)}

\subsubsection{Structures and composition of biological thiols}

Biothiols such as homocysteine (Hcy), glutathione (GSH) and cysteine (Cys), (structures shown in Figure 1.1) play significant roles in regulating various biological processes. GSH, a tripeptide $\gamma$-glutamyl-cysteinyl-glycine is the major non-protein intracellular thiol which apart from being an important antioxidant, also regulates cellular functions such as gene expression, cell proliferation and apoptosis. ${ }^{1-3}$ GSH concentrations in cells range from $1-10 \mathrm{mM}$ whereas plasma levels range from 1-6 $\mu \mathrm{M} .^{1,4-7}$ Cys is the most abundant low-molecular-weight thiol in plasma and plays a fundamental role in protein synthesis, cell metabolism and detoxification. ${ }^{8}$ It is a precursor of GSH and its structure closely resembles that of Hcy. Healthy levels of Cys range from 135-300 $\mu \mathrm{M}$ and its abnormal levels are associated with various disease states such as liver damage, edema and skin lesions. ${ }^{7,9-11}$

Hcy is a metabolic intermediate derived from the essential amino acid methionine in the biosynthesis of Cys. ${ }^{12}$ Structurally, Hcy differ from Cys by a single methylene group (Figure 1.1) which makes their individual determination very challenging. It exists in various forms in plasma with the most dominant one being the protein bound accounting for $70-80 \%$ of total Hcy, followed by the free oxidized form (non-proteinbound disulfides) constituting $20 \%$ and finally free, reduced Hcy making up less than 
$1 \%$ of the total pool. ${ }^{7}$ Plasma total homocysteine (tHcy) refers to the sum of all the three forms (all circulating disulfides and reduced Hcy).

Normal plasma tHcy concentrations range from 5-15 $\mu \mathrm{M}$. At elevated levels, a condition known as hyperhomocysteinemia occurs that is an independent risk factor for several major pathologies such as cardiovascular disease (CVD), dementia, osteoporosis and Alzheimer's disease. ${ }^{13-15}$ Hyperhomocysteinemia is classified into three categories including moderate $(15-30 \mu \mathrm{M})$, intermediate $(30-100 \mu \mathrm{M})$ and severe $(>100 \mu \mathrm{M}))^{7,16}$ Due to the relationship between hyperhomocysteinemia and lower methionine synthase activity and folate deficiency, it has been proposed that folate therapy may be useful for for hyperhomocysteinemia-related disorders. ${ }^{17}$<smiles>N[C@@H](CCS)C(=O)O</smiles>

Hcy 1<smiles>N[C@@H](CS)C(=O)O</smiles>

Cys

2<smiles>N[C@@H](CCC(=O)N[C@@H](CS)C(=O)NCC(=O)O)C(=O)O</smiles>

GSH

Figure 1.1. Structures of selected biological thiols.

\subsubsection{Significance of Hcy to public health}

Hcy plays a critical role in methionine and folate metabolism. It is a metabolic intermediate that participates in two pathways, the re-methylation (methionine cycle) and transsulfuration (folate cycle, Figure 1.2). ${ }^{18,19}$ In the re-methylation pathway, Hcy 
receives a methyl group from 5-methyltetrahydrofolate (most common in cells and tissues) or betaine and is reconverted to methionine, which is fundamental to biochemical processes such as protein and nucleic acid synthesis. In the transsulfuration pathway, Hcy is converted to Cys through cystathionine in a reaction involving serine and catalyzed by a vitamin B6 dependent enzyme, cystathionine- $\beta$-synthase. This pathway is irreversible indicating the inability of Cys to serve as a precursor for methionine. Most Cys formed is in turn converted to the tripeptide GSH.

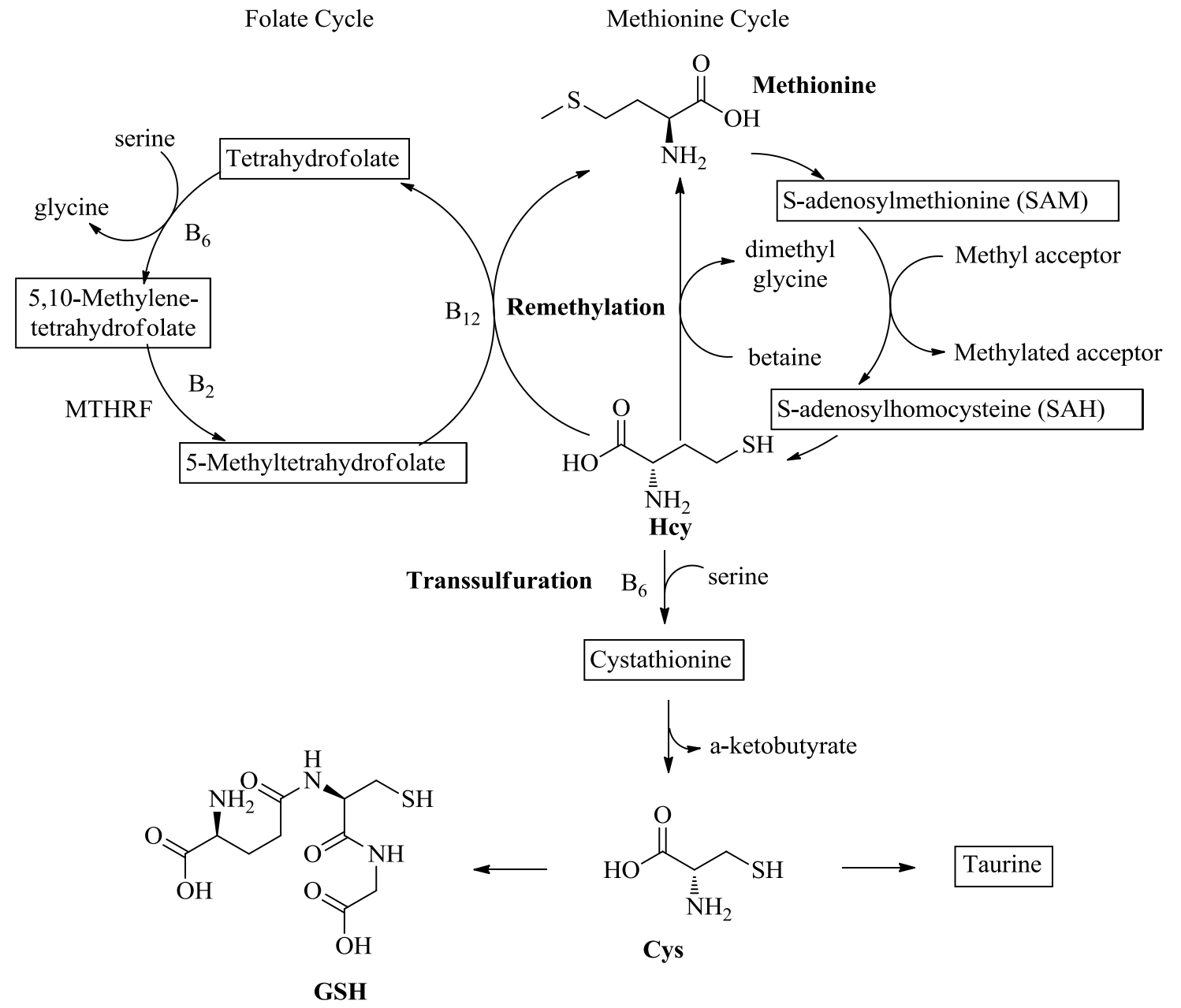

Figure 1.2. Pathways for the metabolism of Hcy. ${ }^{18,19}$ 
Elevated plasma levels of Hcy ${ }^{20,21}$ can be caused by metabolic defects in the two pathways or deficiencies of enzymes and cofactors required in these pathways. Hyperhomocysteinemia can be caused by the impaired synthesis of 5methyltetrahydrofolate due to the deficiency of folate or a defect in methylenetetrahydrofolate reductase (MTHFR). ${ }^{12}$ In these cases, Hcy meant for remethylation would be channeled to the transsulfuration pathway thereby decreasing the intracellular s-adenosylmethionine (SAM) concentration as well as overburdening the transsulfuration system with excess Hcy. The additional Hcy coupled with lower concentrations of SAM (not sufficient to activate cystathionine synthesis) damage the transsulfuration pathway. Eventually, Hcy accumulates in cells and is consequently exported into the blood causing hyperhomocysteinemia. ${ }^{12,19,22}$

Another cause of hyperhomocysteinemia is a defect in cystathionine- $\beta$-synthase that impairs the transsulfuration pathway via the diversion of Hcy towards the remethylation pathway. This increases the rate at which methionine is synthesized leading to a rise in the level of intracellular SAM. The level of SAM can reach a point where it causes feedback inhibition of MTHFR, thus blocking the re-methylation process. This leads to severe hyperhomocysteinemia because both pathways would have been impaired. $^{12,22}$ Abnormalities in the enzyme cystathionine- $\beta$-synthase also cause homocystinuria which is an autosomal recessive disorder characterized by ocular lens dislocation, mental retardation and aggressive vascular disease, in particular venous thromboembolism. ${ }^{18}$ Other factors which have been reported to cause hyperhomocysteinemia include environmental toxins as well as poor nutrition and 
lifestyle. For example, cigarette smoking, a high intake of alcohol and caffeine are associated with increased Hcy levels. ${ }^{23}$ Hcy concentrations rise in diseases such as cancer, hypothyroidism, inflammatory bowel and end-stage renal disease. ${ }^{20,24,25} \mathrm{Hcy}$ levels rise with age, and women generally have lower levels than men but the concentrations rise after menopause. $^{26,27}$

Several studies have been published showing an association between elevated levels of Hcy and CVD. A strong link between plasma Hcy and CVD mortality has been established in an early study by the World Health Organization (WHO) ${ }^{28}$ Nygard, et al., in their 2001 study, discovered that the cardiovascular related mortality ratios doubled when Hcy levels were twice as high. ${ }^{29}$ Meta-analyses of both prospective and genetic studies demonstrated that there is a significant association between Hcy levels and CVD. In these studies it was predicted that lowering tHcy by $3 \mu \mathrm{mol} / \mathrm{L}$ (by increasing folic acid intake) would reduce the risk of heart disease by $11-16 \%$, thrombosis by $25 \%$ and stroke by $19-24 \% .^{30,31}$ A recent study by Bertoia, et al., found that higher Hcy levels were positively associated with a risk of peripheral artery disease in men. ${ }^{32}$ It has been reported that each $5 \mu \mathrm{mol} / \mathrm{L}$ increase in Hcy level increases the risk of coronary heart disease (CHD) events by approximately $20 \%$, independently from traditional CHD risk factors. In the Multi-Ethnic Study of Atherosclerosis (MESA), it was found that higher Hcy levels were significantly associated with a higher incidence of CVD and CHD. ${ }^{33}$

Meta-analysis genetic studies have also given further evidence showing the casual relationship between elevated tHcy and CVD. The homozygous mutant for MTHFR C677T polymorphism which affects about $10 \%$ of individuals worldwide results in 
impaired folate metabolism and elevated tHcy levels. ${ }^{34}$ Three meta-analyses involving over 25,000 cases examined the impact of this genetic variant (homozygous MTHFR polymorphism) on heart disease risk and found that the risk was higher in individuals with polymorphism compared to those without the polymorphism by about $14-21 \%$. $^{30,3536}$ In another study, the relationship between MTHFR polymorphism and the severity of coronary artery disease in patients undergoing coronary artery bypass surgery showed that Hcy levels were significantly higher in patients with coronary artery disease than in control subjects. ${ }^{37}$

Hcy has also been associated with neural tube defects, increased incidence of dementia, inflammatory bowel disease and osteoporosis. ${ }^{38,39}$ In addition, elevated Hcy can be used to diagnose and follow the progression of cardiovascular disease in cases where classical risk factors such as hypertension, diabetes and hyperlipidemia are absent. Therefore a compelling case can be made for detecting and treating hyperhomocysteinemia in patients with a history of premature cardiovascular disease, stroke, or venous thromboembolism. ${ }^{40}$

\subsection{Hcy controversy}

Although significant research has shown that Hcy is a risk factor at even modestly elevated levels, ${ }^{41}$ the role of Hcy in cardiovascular diseases is not clear and has been debated for decades as to whether it is just a biomarker or actually a risk factor. However, according to the 2006 government study published in Circulation by Yang, Q., et al., 
strong evidence was provided showing the association between Hcy and cardiovascular risk. $^{42}$ They found that folate fortification (for lowering Hcy levels) significantly reduced deaths from strokes and heart attacks. More recent studies confirmed that homocysteine is a strong, independent CVD predictor. ${ }^{43}$ More so, Wang et al., in their meta-analysis on the efficacy of folic acid supplementation in stroke prevention concluded that folic acid supplementation can effectively prevent stroke in populations with no or partial folic acid fortification. $^{44}$

On the other hand, a meta-analysis of the Cochrane Database of Systematic Reviews published by Carvajal et al., 2009 indicated that Hcy lowering therapy did not reduce the risk of myocardial infarction and stroke. ${ }^{45}$ In another recent study, it was concluded that folic acid based homocysteine lowering does not reduce cardiovascular events in people with kidney disease. ${ }^{46}$ Numerous secondary intervention trials involving lowering Hcy levels by vitamin supplementation have been performed recently but none afforded conclusive evidence of generally improved CVD outcomes. ${ }^{47,4849,50}$ However, it should not be assumed that no relationship exists because such studies conflict with a massive body of published evidence clearly showing that Hcy is toxic at even modestly elevated levels. $^{47,51}$ They contradict other clinical trials (e.g., the NIHLB's Framingham study) in the broader CVD biomarker field that view Hcy monitoring as useful, for instance, in clinical situations wherein CVD patients are asymptomatic and/or traditional risk factors are not elevated or had been already well-controlled. ${ }^{52}$

Several explanations for the intriguing and conflicting clinical trial outcomes have been reported and these include: ${ }^{47,53}$ 
(i) homocysteine-lowering trials could have been underpowered by the duration of follow-up which has been too short as shown in the meta-analysis of the effect of B vitamins on stroke. ${ }^{54}$ The result of this study was a full absence of any effect in the short-term but a statistically significant $29 \%$ reduction in the studies with at least 36 months of follow-up;

(ii) vitamin supplements given to lower Hcy levels have themselves been shown to exacerbate CVD, and therefore negated any benefits of Hcy lowering;

(iii) cohorts recruited all had advanced atherosclerotic disease and thus Hcy had apparently already done its major damage in mediating progression, rendering lowering Hcy interventions too late;

(iv) since elevated Hcy levels can arise from and impact a highly complex set of pathogenic mechanisms, more specifically targeted mechanism- and/or patient-specific interventions than relatively simple vitamin supplementation may be needed to effectively manage CVD via targeting Hcy levels;

(v) interpretation of the results from these large vitamin trials may have been incorrect due to statin/aspirin therapy, which was applied along with the vitamin and folic acid fortification. 
Despite this controversy, the consistency in published evidence showing a strong relation between hyperhomosysteinemia and cardiovascular related diseases renders Hcy determination of clinical significance. ${ }^{47}$ 


\section{CHAPTER 2: HCY DETECTION METHODS}

\subsection{Current clinical methods}

The clinical relevance of Hcy as a diagnostic risk factor for CVD has justified the need to develop rapid, precise, and accurate techniques for detecting and quantifying tHcy. Currently, there are three main tHcy testing methods that are used in clinical laboratories including chromatographic (analytical HPLC with a derivatization step, gas chromatography/mass spectrometry (GC-MS)), immunoassays and enzymatic assays. 38,41,55,56 While the HPLC method has high precision and sensitivity, it often requires skilled staff, is labor intensive and throughput may be low. ${ }^{38,57}$ The need for derivatization introduces additional sample processing steps hence it's not the method of choice in major clinical laboratories. GC-MS uses complex instrumentation that is not typically applied at point-of-care and requires tedious procedures not suitable for routine diagnostic applications.

Immunoassays and enzymatic methods use antibodies and biomolecules that are relatively expensive and fragile; therefore, careful attention to storage conditions is required. ${ }^{58}$ Despite those shortcomings, the enzyme cycling assay is becoming the preferred method for clinical laboratories involved in routine screening applications over the immunoassays and chromatographic approaches because it uses less reagents, does not require sample pre-treatment and offers high throughput. ${ }^{58,59}$ Hcy assay interference from hemolysis, icterus, and lipemia has been reported with the hemoglobin interference being the most prevalent. ${ }^{60}$ Large differences between the methods used and the different 
testing laboratories have been reported creating the need for more standardization of tHcy testing methods. ${ }^{55,61,62}$

\subsection{Optical methods}

Optical techniques including colorimetric or fluorescence and luminescent have received increased attention over other analytical methods because they offer a number of advantages such as operational simplicity, low cost, suitability for monitoring

intracellular analytes and high sensitivity. ${ }^{63,64} \mathrm{~A}$ wide variety of useful optical detection probes for biological thiols based on different mechanisms has been reported. ${ }^{65-67}$ Most have no specificity for Hcy over other structurally related analytes such as Cys and GSH. Selective determination of plasma tHcy levels (normal, 5-15 $\mu \mathrm{M}$ ) is further complicated by the higher levels of Cys $(135-300 \mu \mathrm{M})$ almost 20 times that of Hcy and comparable levels of GSH $(1-6 \mu \mathrm{M}) .{ }^{6,7,10,16}$

\subsubsection{Hcy/Cys selective probes over GSH}

Most probes can distinguish Cys/Hcy from GSH and recent examples include a commercially available chromophore, 4-chloro-7-nitro-2,1,3-benzoxadiazole (NBD-Cl) reported by Niu and co-workers (Figure 2.1). ${ }^{68}$ The proposed mechanism involves intramolecular displacement of chloride by thiolate followed by replacement of the thiolate with the amino groups of Cys/Hcy, but not GSH to form amino-substituted 
products which show strong fluorescence compared to that exhibited by the sulfursubstituted product for GSH.

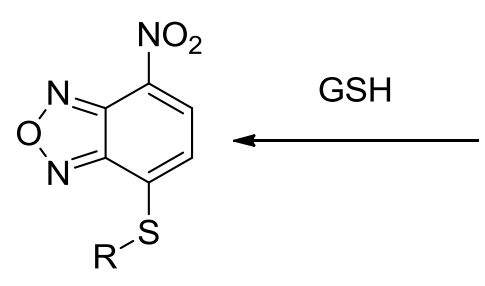

6

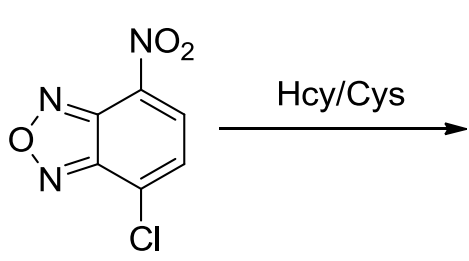

4<smiles>O=C(O)C([As])Nc1ccc([N+](=O)[O-])c2nonc12</smiles>

5

Figure 2.1. Substitution reaction of NBD-Cl (4) with aminothiols. ${ }^{68}$

Goswami et al., developed a turn on excited-state intramolecular proton transfer (ESIPT) probe, 5(benzothiazol-2-yl)-4-hydroxyisophthalaldehyde (BHI) for rapid and ratiometric fluorogenic detection of Hcy and Cys over other amino acids (Figure 2.2). ${ }^{69}$ This probe, however cannot discriminate between Hcy and Cys.<smiles>O=Cc1cc(C=O)c(O)c(-c2nc3ccccc3s2)c1</smiles>

7

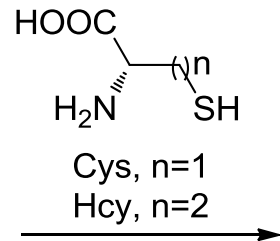

$\mathrm{HOOC}$<smiles>O=Cc1cc(-c2nc3ccccc3s2)c(O)c(C2NC(C(=O)O)CS2)c1</smiles>

8

Figure 2.2. Reaction of 7 with Hcy/Cys. ${ }^{69}$ 


\subsubsection{Hcy selective probes}

Though some chemosensors or chemodosimeters that selectively respond to Hcy over Cys and other thiols have been reported, they are typically tested at equimolar, rather than more natural $c a$. 20-fold excess Cys concentrations. ${ }^{70-72}$ A phosphorescent sensor based on the iridium(III) complex was reported by Chen and co-workers to be selective for Hcy over Cys and GSH (Figure 2.3). With this probe phosphorescence emission is enhanced in the presence of Hcy and quenched in the case of Cys. Thus, the aldehyde group on this probe reacts with both Hcy and Cys forming thiazinane and thiazolidine adducts respectively. However, the basis for Hcy selectivity was reported to be a result of a photoinduced electron-transfer process on the thiazolidine (Cys adduct). While it's a promising probe, higher concentrations of Hcy $(400 \mu \mathrm{M})$ were used, by far more than physiological levels. A higher percentage (90\%) of the organic solvent (DMSO) was used which would affect the accurate quantitation of analyte in biological samples. $^{73}$



9
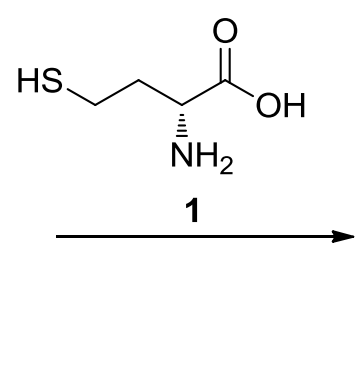

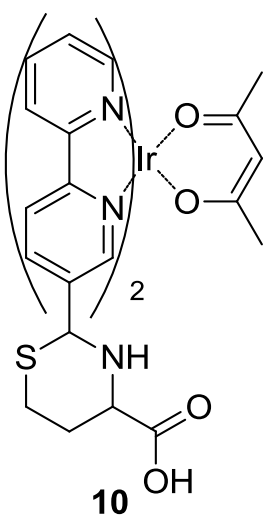

Figure 2.3. Possible recognition mechanism of 9 with Hcy. ${ }^{70}$ 
Yu et al., developed a fluorescent mesoporous silica nanoparticle (MSN) sensor with an anthracene nitroolefin as well as the polyethylene glycol (PEG) covalently immobilized on the particle surface. ${ }^{71}$ While all thiols react with the nitroolefin moiety in a Michael addition reaction restoring fluorescence of the anthracene (Figure 2.4), the basis for Hcy selectivity was the PEG molecules that served as a selective barrier blocking other thiols from diffusing into the nanopores. The reported selectivity was achieved at equimolar amounts of thiols $(50 \mu \mathrm{M}), 3$ times more than plasma levels of tHcy and 6 times less than Cys plasma levels indicating that at physiological levels, Cys would become a major interference. Moreover, at $50 \mu \mathrm{M}$, it was evident that Cys showed responses that were higher than the control.

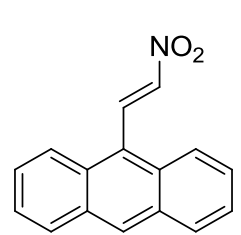

11
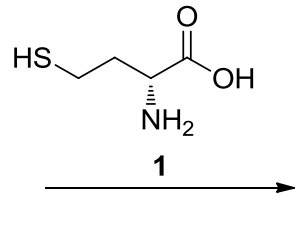

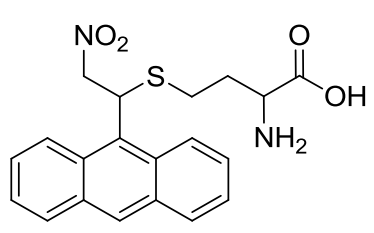

12

Figure 2.4. Proposed mechanism for Hcy selectivity by the Mesoporous silica nanoparticle - based sensor. ${ }^{71}$

Recently, Peng and co-workers reported a redox-based fluorescent probe for selective determination of Hcy (Figure 2.5). ${ }^{72}$ The proposed reaction mechanism involves the oxidation of Hcy by a dansyl azide-2 (DN-2) probe selectively over Cys because of the favorable formation of cyclic five-membered ring intermediate as opposed to the strained four membered ring for Cys. The reported selectivity was obtained using equimolar amounts of $100 \mu \mathrm{M}$ for both Hcy and Cys that do not represent the 
physiological levels. Additionally, at $200 \mu \mathrm{M}$ Cys was found to be interfering with Hcy determination, elevating fluorescence response of probe to Hcy by $\sim 40 \%$.<smiles>CN(C)CCN(C)c1cccc2c(S(N)(=O)=O)cccc12</smiles>

13



14

Figure 2.5. Sensing reaction of $\mathbf{1 3}$ with Hcy. ${ }^{72}$

Our group developed a visual detection method for Hcy and Cys with aldehydebearing fluorophores based upon the well-known reaction of these thiols to form heterocyclic thiazolidines and thiazinanes. ${ }^{74}$ Fluorescence quenching of fluorescein aldehyde probes following the formation of thiazolidine/thiazinane heterocycles with Cys and Hcy was observed under the basic conditions investigated. We further improved this method recently to selectively detect Hcy over Cys by tuning $\mathrm{pH}$ and excitation wavelength. $^{75}$ At lower $\mathrm{pH}$ (5.5-6) and longer wavelength excitations (495 nm), fluorescence enhancement of $>5$ fold for Hcy over Cys is achieved through inhibition of photo induced electron transfer (PET) as the thiazolidine/thiazinane heterocycles formed become protonated (Figure 2.6). 




Figure 2.6. Mechanism of signal transduction in generic aldehyde-bearing amino thiol probes. (a) PETbased fluorescence quenching following reaction of Cys/Hcy with the aldehyde group. (b) PET inhibition through hydrogen bonding between the amine-containing heterocycle and adjacent groups. (c) PET inhibition through protonation of the amine-containing heterocycle. ${ }^{75}$

\subsubsection{Hcy selective probes based on free radical chemistry}

Biothiols are oxidized to thiyl radicals via three main routes including reaction with carbon centered radicals, with reactive oxygen species (ROS) and through enzymatic oxidation by peroxidases. ${ }^{76}$ Two important reactions of thiyl radicals include the reaction with the thiolate to form a reducing disulfide radical anion (Equation 1) and the formation of a reducing alpha-amino carbon-centered radical (Equation 2).

$$
\begin{gathered}
\mathrm{RS}^{-}+\mathrm{RS}^{\bullet} \rightleftharpoons \mathrm{RSSR}^{\bullet-} \\
\mathrm{RS}^{\bullet}+\mathrm{HCCR}_{\mathrm{I}} \rightleftharpoons \mathrm{RSH}+{ }_{\mathrm{l}}^{\mathrm{C} R}
\end{gathered}
$$


Rate constants of Eq 2 have been reported to be generally of the order of $10^{8} \mathrm{M}^{-1} \cdot \mathrm{s}^{-1}$ but the reverse reaction is much slower. ${ }^{76,77}$ If the hydrogen donation reaction in equation 2 , known as the "repair reaction", proceeds intermolecularly, it can promote the "repair" of carbon-centered free radical-containing biomolecules such as carbohydrate-based radicals of DNA. ${ }^{76}$ Following the report by Neta and co-workers of the $\alpha$-amino alkyl radical of Cys, Sjoberg et al. and Zhao et al. extended the work and reported the formation of reducing $\alpha$-amino alkyl radicals of GSH and Hcy through intramolecular rearrangement of their respective thiyl radicals. ${ }^{78-80}$

Importantly, Zhao and co-workers reported that intramolecular formation of the captodatively-stabilized $\alpha$-amino alkyl radical in Hcy should be favorable. This was attributed to a kinetically-favored intramolecular hydrogen atom transfer (HAT) mechanism, involving the favored formation of a 5-membered ring in the transition state, as opposed to 4- and 9-membered ring configurations for Cys and GSH, respectively (Figure 2.7). Alpha-amino acid carbon radicals are well-known strong reductants. Captodative stabilization of the alpha-radical renders the alpha $\mathrm{C}-\mathrm{H}$ bond weaker than the S-H bond by approximately $4 \mathrm{Kcal} / \mathrm{mol}$. 


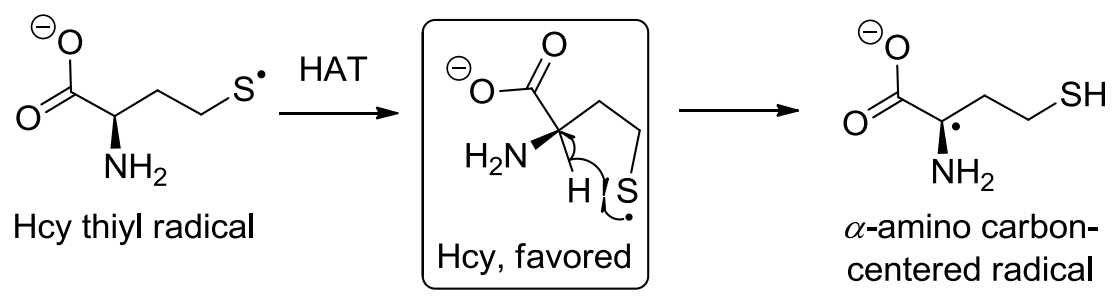

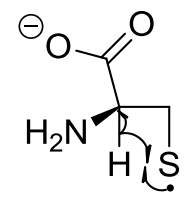

Cys, unfavored

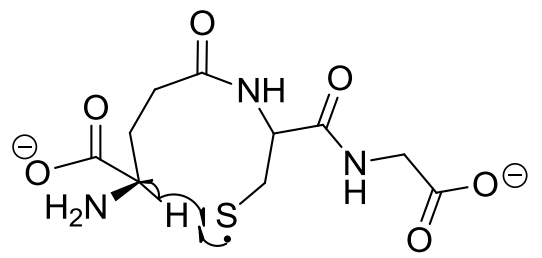

$\mathrm{GSH}$, unfavored

Figure 2.7 Kinetically favored HAT reaction for Hcy. The rate constant for the kinetically-favored sulfurto-carbon-H atom transfer for Hcy is $>10^{5} \mathrm{~s}^{-1} .80$

In their study, Zhao and co-workers used the azide radical to oxidize thiols and the formation of reducing radicals was monitored via UV-Vis absorption spectra of the reduced methyl viologen radical cation $\left(\mathrm{MV}^{\circ}\right)$. Under the highly basic conditions $(\mathrm{pH}$ 10.5) investigated by Zhao et al., no colorimetric selectivity between GSH, Cys and Hcy was observed. This was due to the presence of significant amounts of thiolate anion promoting the formation of a reducing disulfide radical anion (equation 1) that also reacts with methyl viologen $\left(\mathrm{MV}^{2+}\right)$ independently of the HAT mechanism. Conversely, neutral conditions investigated by us diminish thiolate formation such that there is only a relatively small percentage of deprotonated thiol. Thus, it was reasoned by our group, that the Hcy-favored HAT process occurring in equation 2 should be more predominant than interfering disulfide radical anion formation at physiological $\mathrm{pH}$. This enabled us to selectively detect Hcy in human blood plasma without any interference from Cys, GSH and other amino acids in $2004 .^{74,81}$ 
In this colorimetric method remarkable visual color changes were later obtained in human blood plasma without any deproteinization using commercially available $\mathrm{MV}^{2+}$ at neutral $\mathrm{pH}$ and reflux conditions (Figure 2.8). The mechanism involves the reduction of $\mathrm{MV}^{2+}$ by the Hcy- $\alpha$-amino carbon centered radical to its corresponding radical cation which is responsible for the blue color (Figure 2.9). A protocol for visual detection of Hcy was developed based on this method wherein the Hcy thiyl radical is generated by heat. ${ }^{82}$ No washing steps, biological reagents or sample pre-processing steps apart from disulfide reduction are needed in this method.



Figure 2.8. Selective detection of Hcy in human plasma spiked with $4.0 \mathrm{mM}$ of Cys, Hcy and GSH, 8.0 $\mathrm{mM} \mathrm{MV}{ }^{2+}, 0.5 \mathrm{mM}$ Tris buffer $\mathrm{pH} 7.5$, and refluxed for $5 \mathrm{~min}$. Inset shows samples before heating. *Adapted with permission from the Journal of the American Chemical Society, 2005, Volume 127, pages 15949-15958; Weihua Wang, Oleksandr Rusin, Xiangyang Xu, Kyu Kwang Kim, Jorge O. Escobedo, Sayo O. Fakayode, Kristin A. Fletcher, Mark Lowry, Corin M. Schowalter, Candace M. Lawrence, Frank R. Fronczek, Isiah M. Warner, and Robert M. Strongin; Detection of Homocysteine and Cysteine. Copyright 2005 American Chemical Society. 
<smiles>NC(CC[Hg])C(=O)[O-]</smiles>

(a) $\|$ HAT

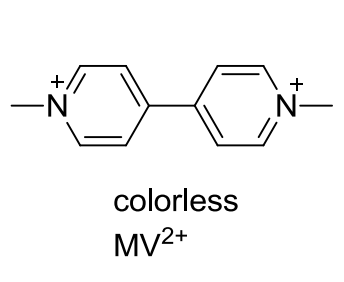

19 (b)

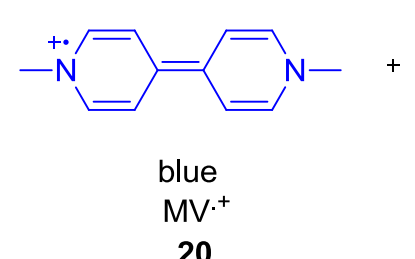

20<smiles>N[C@H](CCS)C(=O)[O-]</smiles>

(c)<smiles>N[C@@H](CCS)C(=O)[O-]</smiles>

Figure 2.9. Reaction mechanism for selective detection of Hcy using $\mathrm{MV}^{2+}$ at neutral $\mathrm{pH}^{74}$. (a) Hydrogen atom transfer (HAT) (b) reduction of $\mathrm{MV}^{2+}$ (c) iminium formation.

While significant progress was made with $\mathrm{MV}^{2+}$, the probe was only applied to non-reduced plasma and had not been tested in real samples. Human plasma had been spiked with commercial thiol samples. To detect total Hcy (tHcy) a new step had to be introduced, reduction step, to convert all oxidized Hcy species into the reduced form since almost $99 \%$ of Hcy is in oxidized disulfide form. Figure 2.10 shows a summary of an improved thermal method that I developed which incorporated the reduction step.

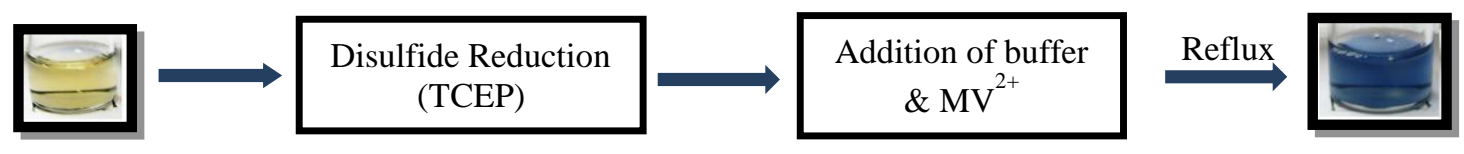

Figure 2.10. Improved thermal method for detection of Hcy.

I investigated this method using non-deproteinized human serum calibration standards (NIST SRM 1955), and successfully distinguished micromolar concentration differences $(3.79,6.13,13.4$ and $38.73 \mu \mathrm{M})$ of tHcy visually using $\mathrm{MV}^{2+}$ (Figure 2.11). ${ }^{66}$ 
As tHcy concentration increased, the color became successively darker and darker as shown by different color shades. The assay protocol involved no sample processing. It only required a two-fold dilution, addition of $\mathrm{MV}^{2+}$ and tris(2-carboxyethyl) phosphine (TCEP), a disulfide reducing agent and 2 min heating at reflux. In this improved method a homogenous solution was formed making it easier to distinguish between different color shades unlike in the previous method where proteins formed clumps upon heating (Figure 2.8).

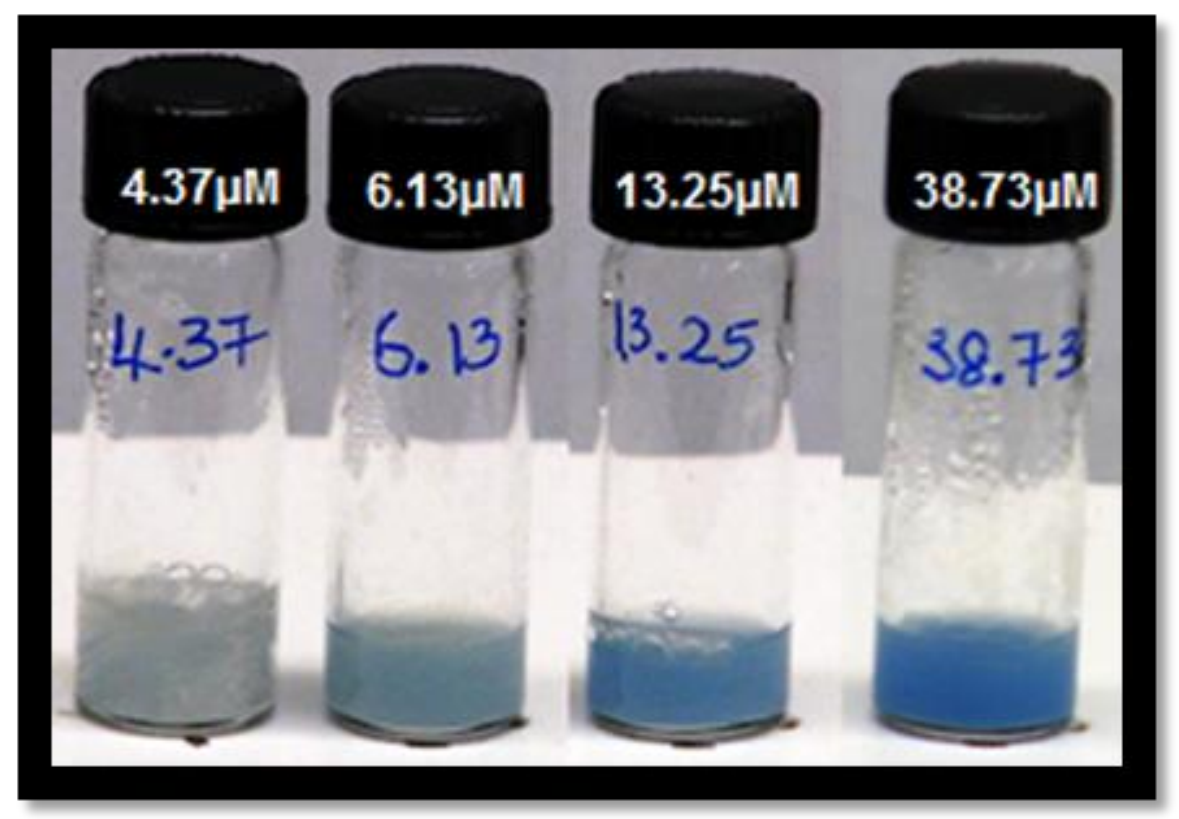

Figure 2.11. Visual detection of Hcy in human serum calibration standards. Left to right: Hcy concentrations: $3.79,6.13,13.4$ and $38.7 \mu \mathrm{M}$ respectively. ${ }^{66}$

In order to make the assay potentially more user friendly and benign for potential application at point of care diagnostics and in home test kits, we continued to improve 
upon the design, synthesis and development of room temperature methods for selective detection of Hcy. Besides the use of heat to promote the reaction, the $\mathrm{MV}^{2+}$ probe is a herbicide and so we investigated the use of less- or non-toxic derivatives. We actually took a two-pronged approach involving the use of photolytic methods described in chapter 3 and the use of novel asymmetric viologen probes described in chapter 4 . 


\section{CHAPTER 3: PHOTOCHEMICAL METHOD FOR SELECTIVE DETECTION OF TOTAL HCY IN PLASMA}

\subsection{Introduction}

The relatively high costs and complexity of the current methods for Hcy detection have intensified the need to develop selective, yet simple and inexpensive methods that can be used at point of care diagnostics and in home test kits to facilitate the diagnosis and treatment of related diseases. Available kits generally use multi-step washing procedures and/or specialized storage below $-20^{\circ} \mathrm{C}$, limiting their use, for instance, in emerging nations with limited access to refrigeration or electricity. Moreover, even in developed countries point-of-care and kit-based assays are of interest considering rising health care costs and increasing interest in patient-based monitoring. ${ }^{83}$

We hypothesized that photolytic methods would afford analogous selectivity via the intramolecular HAT mechanism while enabling the assay to be carried out at room temperature. Johnson and co-workers reported the photochemical reduction of viologens in ethanolic solutions. ${ }^{84} \mathrm{~A}$ mechanism based on the abstraction of a methylene hydrogen atom from $\mathrm{EtOH}$ to form a free radical that reduced the viologen in direct sunlight was proposed. We envisioned that this approach could be compatible with our HAT mechanism for Hcy via photolytic, rather than thermal generation of the Hcy thiyl radical. 


\subsection{Results and discussion}

In exploring the use of photolytic methods to detect Hcy, initially we exposed solutions of thiols and $\mathrm{MV}^{2+}$ in Tris buffer at neutral $\mathrm{pH}$ to direct sunlight at room temperature. A blue color was observed within 1 minute in the Hcy sample while other thiols solutions remained unchanged (Figure 3.1).

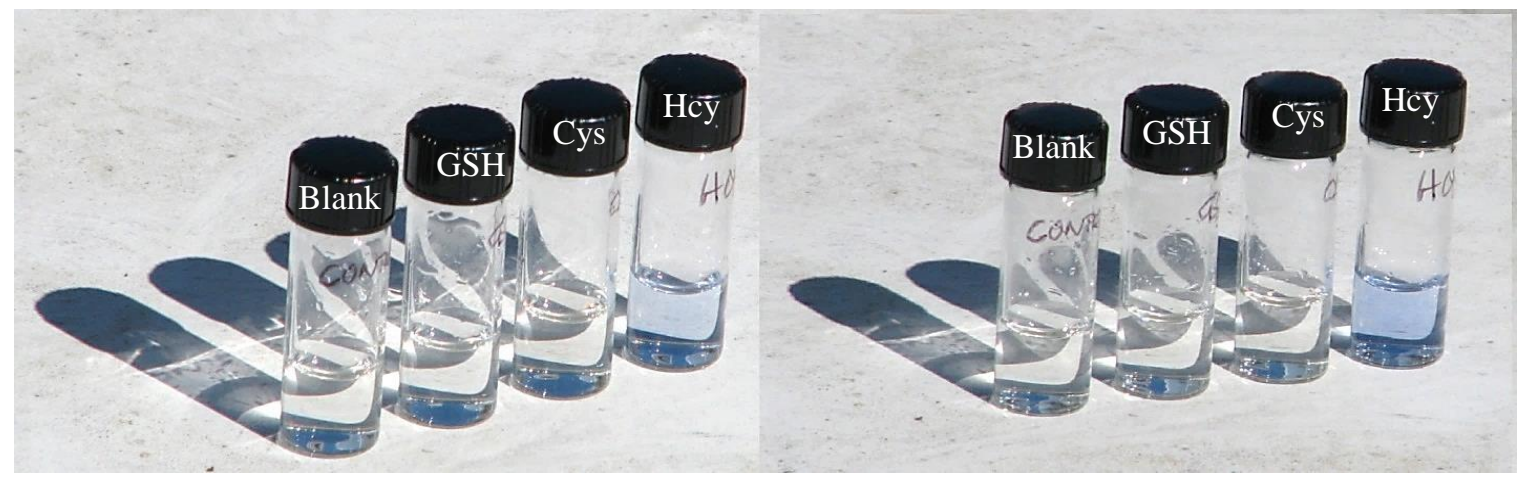

Figure 3.1 Response of $\mathrm{MV}^{2+}$ towards various thiols upon exposure to direct sunlight. Solutions of $\mathrm{MV}^{2+}$ $(50 \mathrm{mM})$ were mixed with thiols $(20 \mu \mathrm{M})$ in $0.5 \mathrm{M}$ Tris buffer at $\mathrm{pH} 7$, saturated with argon and exposed to sunlight. Pictures were taken at $1 \mathrm{~min}$ (left) and $2 \mathrm{~min}$ (right) after exposure to sunlight.

To create a laboratory test, we reasoned that an appropriate light source to generate the thiyl radical should emit around $325 \mathrm{~nm}$ based on the reported $\mathrm{S}-\mathrm{H}$ bond dissociation energy of Cys of $370 \mathrm{~kJ} \mathrm{~mol}^{-1}{ }^{85}$ To this end, we selected a very simple and inexpensive compact fluorescent lamp emitting in this region, Reptisun ${ }^{\mathrm{TM}}$ consisting of $10 \%$ UVB and 30\% UVA. The photolysis experiments were performed using this lamp with a light intensity of $6.85 \mathrm{~mW} \mathrm{~cm}^{-2}$ as measured by a Melles Griot Broadband Power/Energy Meter 13PEM001. We were able to detect Hcy selectively in human blood plasma using $\mathrm{MV}^{2+}$ without any interference from Cys and GSH in the range of their 
physiological concentrations. Upon irradiation of plasma samples spiked with various biothiols, only the Hcy $(15 \mu \mathrm{M})$ spiked sample showed significant absorption response whereas Cys and GSH remained unchanged. The absorption spectra are shown in Figure A1, Appendix A.

In previous studies, benzyl viologen $\left(\mathrm{BV}^{2+}\right)$, a significantly less toxic chromogen than $\mathrm{MV}^{2+}$, was found by our group to be more reactive in forming the Hcy $\alpha$-amino carbon-centered radical than $\mathrm{MV}^{2+}$ under thermal conditions. $\mathrm{BV}^{2+}$ has a less negative reduction potential $(-370 \mathrm{mV})$ than $\mathrm{MV}^{2+}(-446 \mathrm{mV}) .{ }^{86}$ Hence we investigated the response of $\mathrm{BV}^{2+}$ to Hcy and other thiols using the photochemical method. We observed that $\mathrm{BV}^{2+}$ indeed displayed selectivity towards Hcy under the new photolytic conditions compared to structurally related thiols (Figure 3.2). Moreover, changing the chromogen allowed us to lower its concentration from $50 \mathrm{mM}\left(\mathrm{MV}^{2+}\right)$ to $20 \mathrm{mM}\left(\mathrm{BV}^{2+}\right)$. In addition, the Hcy response was greater as compared to photolysis in the presence of $\mathrm{MV}^{2+}$. An irradiation time of just 15 min afforded optimal selectivity and response. 


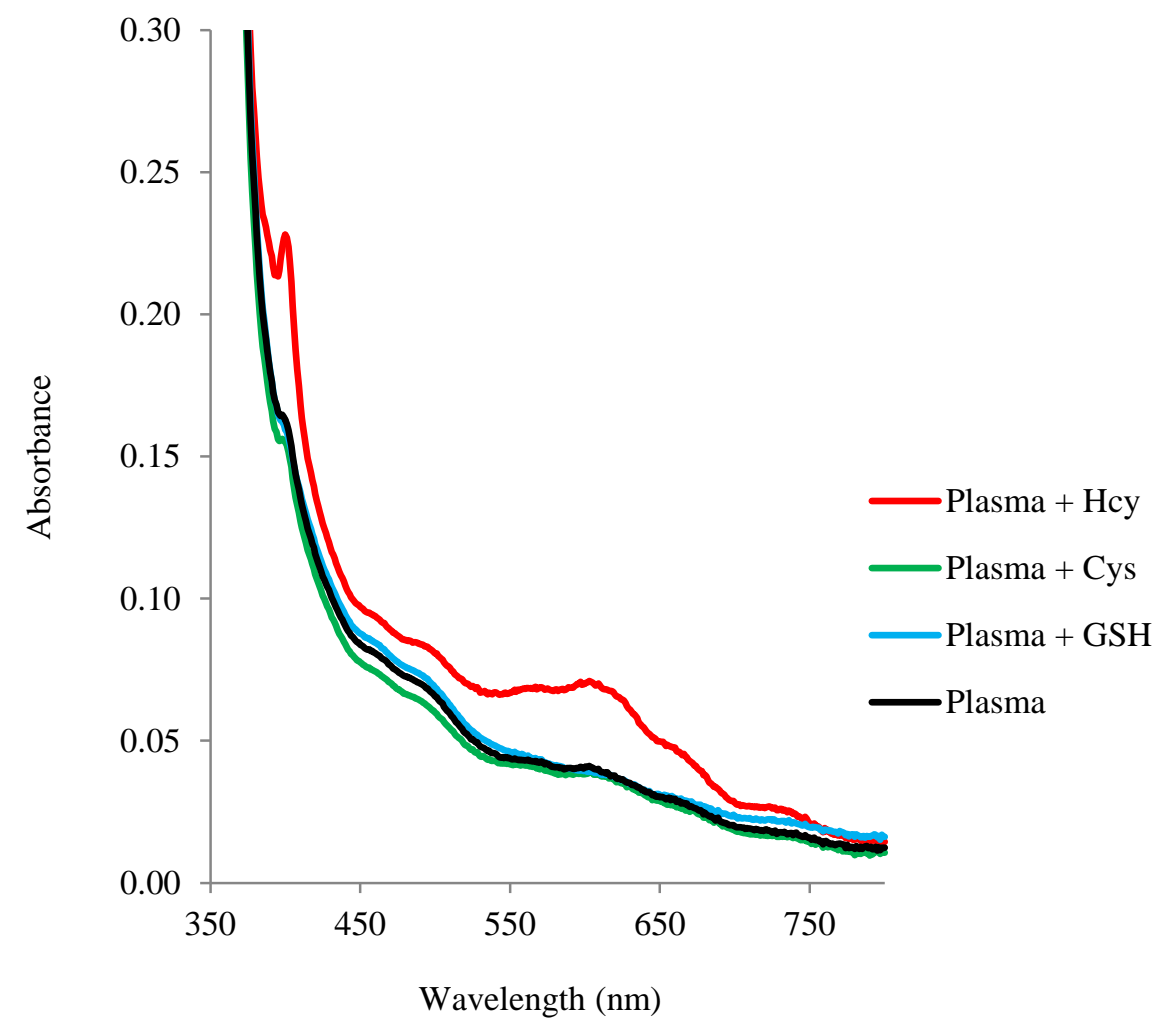

Figure 3.2 Spectral response of $\mathrm{BV}^{2+}$ towards various spiked thiols in human blood plasma upon irradiation. Absorption spectra of solutions of $\mathrm{BV}^{2+}(20 \mathrm{mM})$ in human blood plasma and $0.5 \mathrm{M}$ Tris buffer (pH 7.0) spiked with $1.5 \mu \mathrm{M}$ Hcy, $25 \mu \mathrm{M}$ Cys and $0.6 \mu \mathrm{M}$ GSH. Plasma (10\% v/v) was added to an argonsaturated solution of viologen, thiol \& buffer and irradiated for 15 min using a Reptisun ${ }^{\text {TM }}$ lamp.

Based on the same selectivity pattern and the light source used, the proposed mechanism is assumed to be analogous to the thermal reaction for the selective detection of Hcy we have previously reported. ${ }^{74}$ It involves the generation of the Hcy thiyl radical by photolysis followed by the HAT reaction to form the $\alpha$-amino carbon centered radical that in turn, reduces $\mathrm{BV}^{2+}$ to its corresponding radical cation $\left(\mathrm{BV}^{\circ+}\right)$ responsible for the blue color (Figure 3.3). 


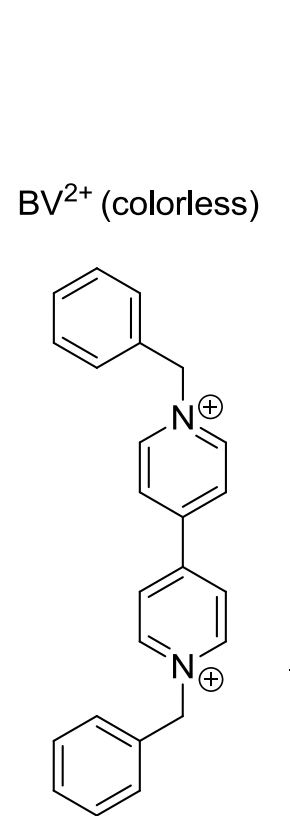

21<smiles>N[C@@H](CCS)C(=O)[O-]</smiles><smiles>N[C@@H](CCS)C(=O)[O-]</smiles><smiles>N[C@@H](CCS)C(=O)[O-]</smiles><smiles>C1CC1</smiles>

1

22

Figure 3.3 Mechanism for the detection of Hcy involving three steps: (i) photolytic generation of Hcy thiyl radical, (ii) hydrogen atom transfer (HAT) turning the thiyl radical into an $\alpha$-amino carbon-centered radical, (iii) reduction of $\mathrm{BV}^{2+} \cdot 83$

Since our ultimate goal was to detect physiological levels of tHcy selectively in human blood plasma, the thiols that are bound to the proteins via disulfide bonds had to be released by reduction. A selective response of $\mathrm{BV}^{2+}$ to $\mathrm{Hcy}$ in human plasma was observed after using immobilized tris(2-carboxyethyl)phosphine (TCEP gel), centrifugation and spiking with various thiols (Figure A2, Appendix A).

We optimized the sample processing by replacing the centrifugation step with a simple filtration of the sample through $0.45 \mu \mathrm{m}$ PVDF filter vials. The spectral response and selectivity was comparable in both methods and excellent selectivity towards tHcy 
was observed (Figure 3.4). Interestingly, the use of filters improved the overall background interference from the plasma components.

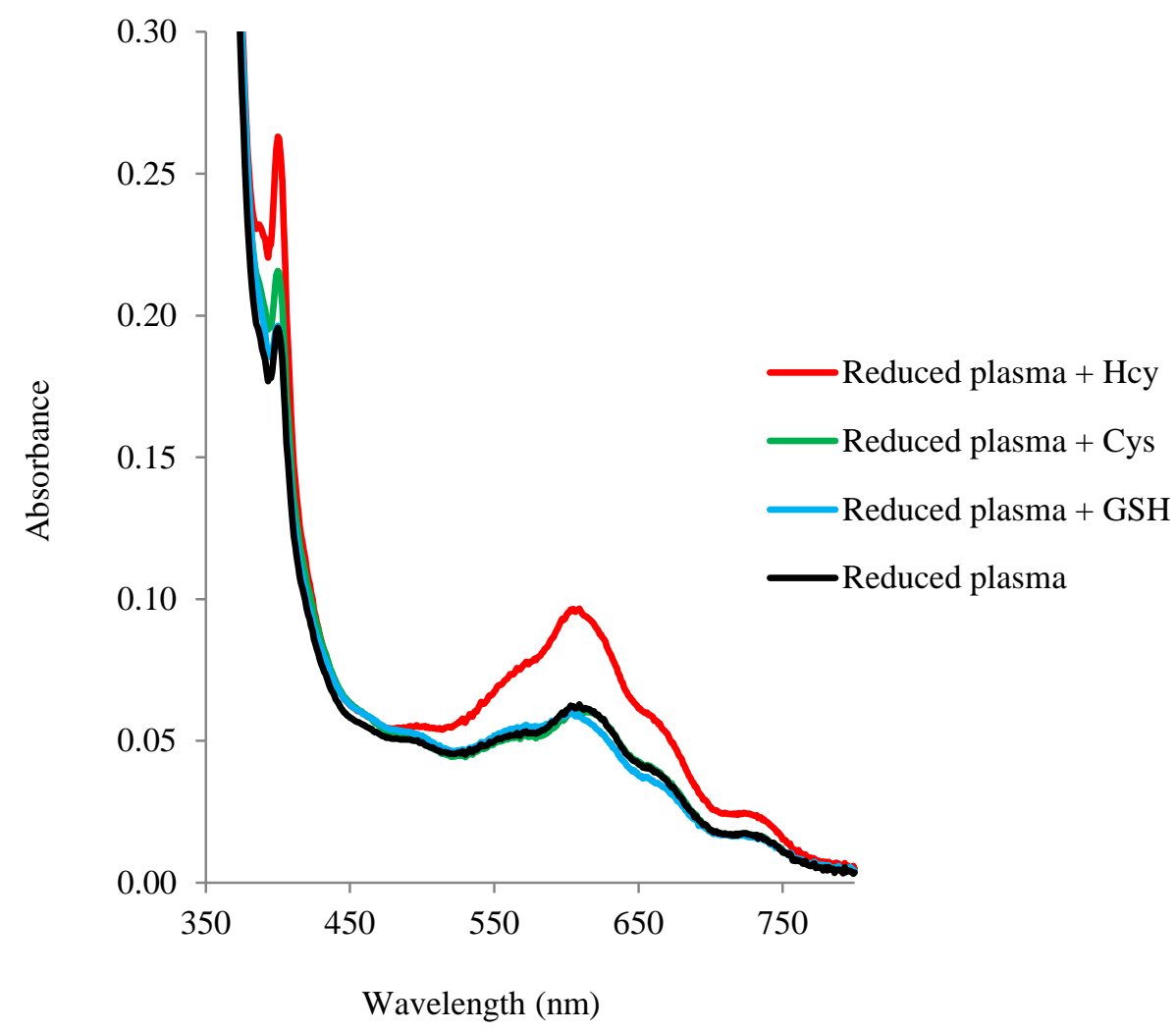

Figure 3.4 Spectral response of $\mathrm{BV}^{2+}$ towards various spiked thiols in reduced human plasma upon irradiation. Absorption spectra of solutions of $\mathrm{BV}^{2+}(20 \mathrm{mM})$ in reduced human blood plasma and $0.5 \mathrm{M}$ Tris buffer ( $\mathrm{pH}$ 7.0) spiked with $1.5 \mu \mathrm{M}$ Hcy, $25 \mu \mathrm{M}$ Cys and $0.6 \mu \mathrm{M}$ GSH. Plasma was incubated for $1 \mathrm{~h}$ with TCEP Gel followed by filtration using a Single StEPTM $0.45 \mu \mathrm{m}$ PVDF filter vial. The reduced, filtered plasma $(10 \% \mathrm{v} / \mathrm{v})$ was added to an argon-saturated solution of viologen, thiol and buffer and irradiated for 15 min using a Reptisun ${ }^{\mathrm{TM}}$ lamp.

The spectral responses of $\mathrm{BV}^{2+}$ to Hcy concentration changes, in spiked reduced human plasma monitored at $615 \mathrm{~nm}$, increased linearly with increasing Hcy concentration over a physiologically relevant concentration range, $0-20 \mu \mathrm{M}$ with a limit of detection of 3.35 $\mu \mathrm{M}$ (Figure 3.5). The inset shows the respective spectral data. This linear 
relationship between absorbance and Hcy concentrations as well as the very low L.O.D makes the assay a potential applicant in the quantitation of tHcy.

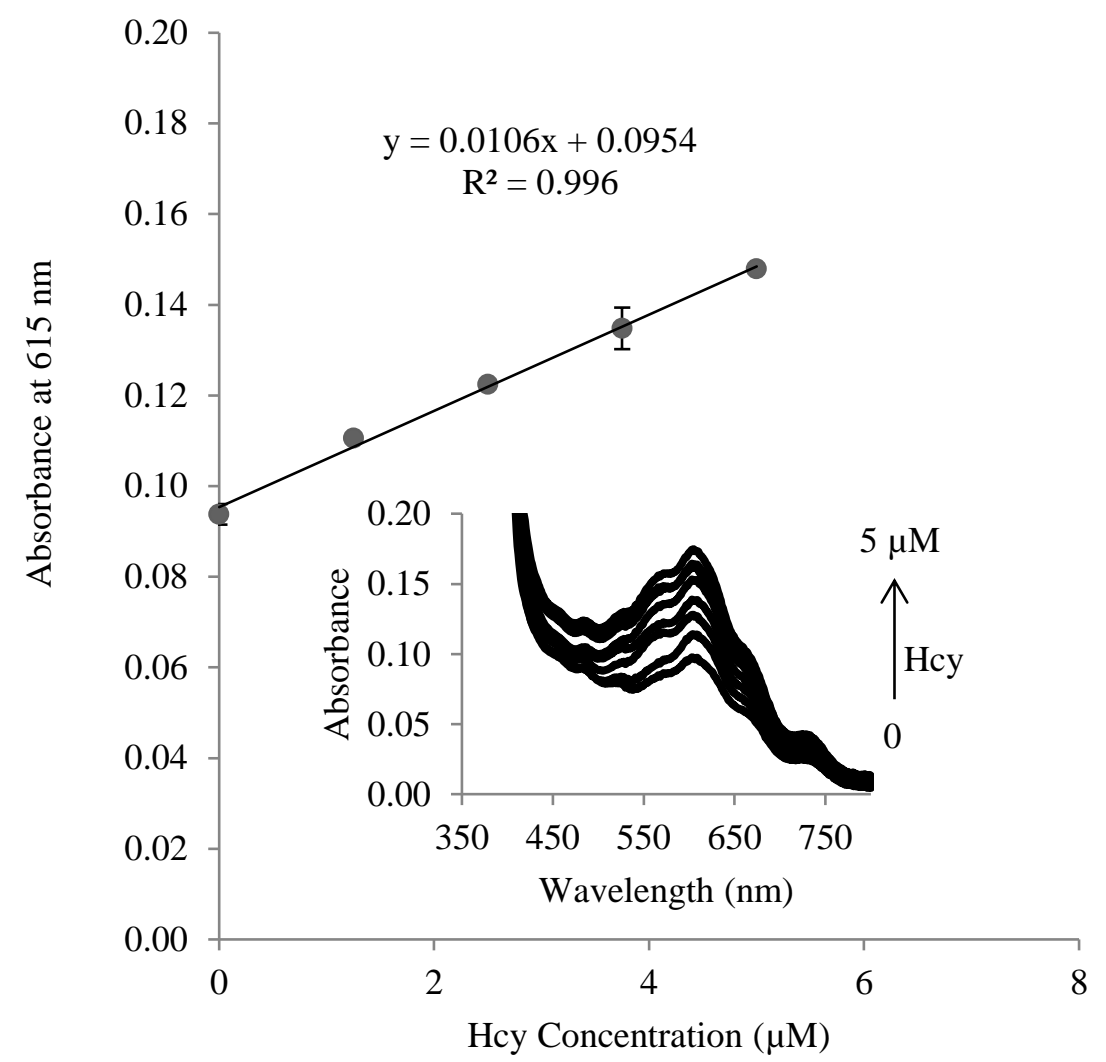

Figure 3.5 Spectral response of $\mathrm{BV}^{2+}$ towards increasing levels of spiked Hcy in reduced human plasma upon irradiation. Absorption spectra of solutions of $\mathrm{BV}^{2+}(20 \mathrm{mM})$ in $25 \%$ human blood plasma and $0.5 \mathrm{M}$ Tris buffer ( $\mathrm{pH} 7.0)$ spiked with $0-5 \mu \mathrm{M}$ Hcy. Plasma ( $25 \% \mathrm{v} / \mathrm{v})$ was added to an argon-saturated solution of viologen, thiol and buffer, and irradiated for 15 min using a Reptisun ${ }^{\mathrm{TM}}$ lamp.

To test the limits of possible Cys and GSH interference with the assay, further experiments were carried out with added excess amounts of Cys and GSH to reduced human plasma solutions. Cys and GSH were found to generate significant response only when their concentrations reach $400 \mu \mathrm{M}$ (almost double the normal concentration in healthy individuals) and $100 \mu \mathrm{M}$ (almost 20 times the normal plasma levels) respectively. 
At these concentrations, their absorbance response was equivalent to that of $5 \mu \mathrm{M}$ Hcy (Figure. A3, Appendix A).

To further evaluate the selectivity of the $\mathrm{BV}^{2+}$ towards Hcy, control experiments using series of other amino acids were performed. Other amino acids produced no significant absorption response as compared to $\mathrm{Hcy}$, further demonstrating that $\mathrm{BV}^{2+}$ is Hcy specific (Figure 3.6).



Figure 3.6 Spectral response of $\mathrm{BV}^{2+}$ towards various spiked amino acids in human plasma upon irradiation. Absorption spectra of solutions of $\mathrm{BV}^{2+}(20 \mathrm{mM})$ in human blood plasma and $0.5 \mathrm{M}$ Tris buffer (pH 7.0) spiked with $1.5 \mu \mathrm{M}$ Hcy and $50 \mu \mathrm{M}$ amino acids (L-Ala, Arg, Gln, Met, Ser, Thr and Phe). Plasma (10\% v/v) was added to an argon-saturated solution of viologen, amino acids and buffer and irradiated for 15 min using a Reptisun ${ }^{\mathrm{TM}}$ lamp. 


\subsection{Experimental section}

\subsubsection{Materials and instrumentation}

All chemicals were purchased from Sigma-Aldrich and Fisher Scientific and used without further purification. The $0.45 \mu \mathrm{m}$ Single StEPTM PVDF filter vials were purchased from Thomson Instrument Company. UV-visible spectra were acquired on a UV-Vis Cary 50 (Agilent Technologies). The light intensity was measured with a Melles Griot Broadband Power/Energy Meter 13PEM001. The light source for the photolysis experiments was a ReptiSun ${ }^{\mathrm{TM}}$ compact fluorescent lamp (Zoo Med Laboratories Inc.).

\subsubsection{Reduction of Human Blood Plasma}

Reconstituted human plasma is incubated with immobilized TCEP gel in a 1:1 volume ratio at room temperature for $1 \mathrm{~h}$ with vortexing. Separation of the reduced plasma from the gel can be achieved by centrifugation at $1000 \mathrm{rpm}$ for $5 \mathrm{~min}$ or by filtration using a Single StEPTM $0.45 \mu \mathrm{m}$ PVDF filter vial.

3.3.3 General procedure for the photolysis of thiols in the presence of viologens

In a quartz cuvette sealed with a rubber septum (GL14-S, Starna Cells), a solution of viologen (20 or $50 \mathrm{mM}$ in $0.5 \mathrm{M}$ Tris buffer $\mathrm{pH} 7.0$ ) is deoxygenated by bubbling $\mathrm{Ar}$ for 5 min. Human plasma (spiked or non-spiked) is added to the cuvette and irradiated for 15 min with a Reptisun ${ }^{\mathrm{TM}}$ lamp. Absorption spectra are collected on a UV-Vis Cary 50. 
The proportion of the plasma solution is $10 \%$ of the total volume after being mixed with the viologen solution.

\subsection{Conclusion}

We have developed a new, relatively simple and inexpensive photochemical assay for the selective detection of total Hcy directly in human blood plasma (Figure 3.7). This method has potential practical application in home test kits or point of care diagnostics because it involves the use of a less toxic chromogen, an inexpensive commercial light source, and simple sample processing, involving only reduction and filtration prior to photolysis and UV-Vis monitoring.



Figure 3.7 Summary of the photochemical method for detection of tHcy. ${ }^{83}$ 


\section{CHAPTER 4: DETECTION OF HCY USING NOVEL ASYMMETRIC VIOLOGEN PROBES}

\subsection{Introduction}

After the success we had with benzyl viologen, we envisioned that viologen compounds with higher reduction potentials than benzyl viologen should be more reactive and would not require either heat or light to promote the reaction. Additionally, in another study conducted by our group in collaboration with Crowe and co-workers, the reduction of viologens by $\alpha$-carbon radicals derived from Hcy and Cys was found to be

$\mathrm{pH}$ dependent. ${ }^{86}$ Symmetric viologens were investigated under reflux conditions and the ones with less negative reduction potentials were more reactive towards amino thiols (Hcy and Cys) at lower $\mathrm{pH}$ (Table 4.1). Moreover, these probes were observed to have a faster onset of color formation and more intense color change when the $\mathrm{pH}$ was increased beyond the endpoint (minimum $\mathrm{pH}$ where color formation was observable).

Based on these findings, we investigated the reaction of phenyl viologen with aminothiols at room temperature. No appreciable reaction was observed and so we were motivated to design viologen probes with even higher reduction potentials. However, there had to be a trade-off between reactivity and selectivity, hence based on the principle that diminished reactivity correlates to higher selectivity, we hypothesized that tuning the redox properties of viologens by making asymmetric probes bearing electron donating and withdrawing groups proximal to the cationic centers would achieve moderate 
reactivity and allow Hcy to be detected selectively at room temperature without the need for heat or light. To this end, five asymmetric viologen probes (Figure 4.1) were synthesized, characterized and screened for detection of Hcy in biological samples.

Table 4.1 Reduction potentials of symmetric viologens and $\mathrm{pH}$ endpoint of Hcy

\begin{tabular}{|l|l|l|l|}
\hline Entry & $\begin{array}{c}\text { Reduction potential, } \\
\text { (Volts) }\end{array}$ & $\begin{array}{c}\text { pH endpoint } \\
\text { of Hcy }\end{array}$ \\
\hline 1 & &
\end{tabular}



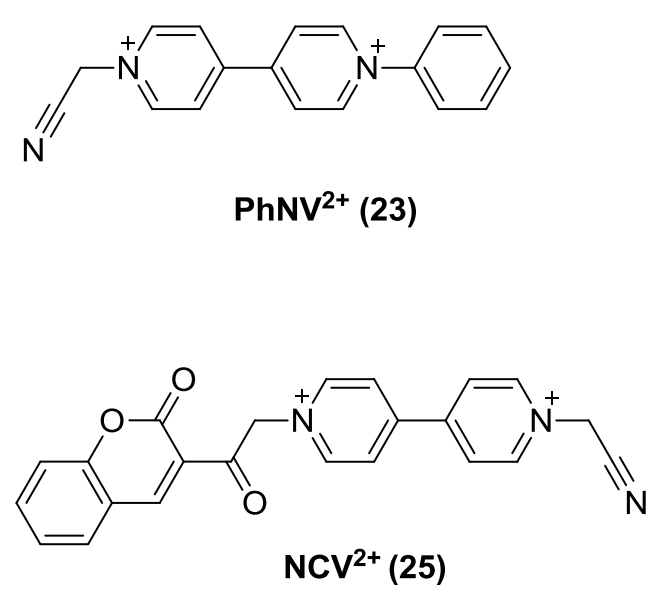


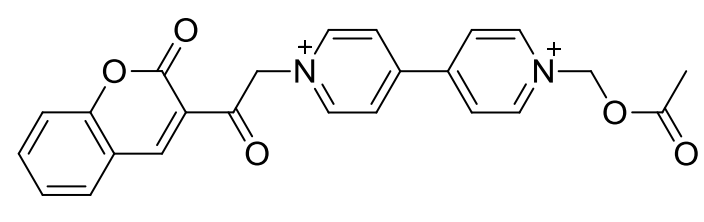

$\operatorname{ACCV}^{2+}(27)$

Figure 4.1 Structures of novel asymmetric viologen probes

\subsection{Results and discussion}

\subsubsection{Cyclic voltammetry studies}

To assess potential electronic effects of different substituents on these asymmetric viologens, cyclic voltammetry studies were carried out in $80 \mathrm{mM}$ phosphate buffer at $\mathrm{pH}$ 8 using glassy carbon as the working electrode. As expected, all five probes were observed to have much less negative reduction potentials compared to methyl viologen and behaved as one step redox system (Table 4.2, Figure A5). This implies that these probes should be easier to reduce and more likely at room temperature without the need 
for heat or light. The trend with increasing reduction potentials (less negative) was found to be: $\mathrm{PhCV}^{2+}(\mathbf{2 4})<\mathrm{PhNV}^{2+}(\mathbf{2 3})<\mathrm{ACNV}^{2+}(\mathbf{2 6})<\mathrm{NCV}^{2+}(\mathbf{2 5})$ as expected, due to the increase in the strength of the electron withdrawing substituents (nitrile, coumarin and ester). Compound $27\left(\mathrm{ACNV}^{2+}\right)$, however, deviated from this trend exhibiting a much lesser negative reduction potential than $\mathrm{ACNV}^{2+}(\mathbf{2 6})$ even though the nitrile or cyano is a more powerful electron withdrawing group than the coumarin. This could have been due to the precipitation we observed in this system and this probe also suffered some adsorption.

At the scan rate of $50 \mathrm{mV} / \mathrm{s}$, viologen probes $\mathbf{2 6}$ and $\mathbf{2 4}$ voltammograms displayed some quasi-reversibility of their reaction systems. This was further confirmed at both higher $(100 \mathrm{mV} / \mathrm{s})$ and lower scan rates $(10 \mathrm{mV} / \mathrm{s})$. The quasi-reversibility may have been a result of slow charge transfer processes or the dication may have been further reduced to its fully reduced state. ${ }^{87}$ It has also been reported that the formation of spin-paired radical cation dimers can cause quasi-reversibility of reduction systems. ${ }^{88}$

Table 4.2: Reduction potentials of novel asymmetric viologens vs methyl viologen

\begin{tabular}{cll}
\hline Entry & Compound & Vs NHE (Volts) \\
\hline 1 & Methyl viologen $\left(\mathrm{MV}^{2+}\right)$ & -0.404 \\
2 & $\operatorname{PhCV}^{2+}(\mathbf{2 4})$ & -0.188 \\
3 & $\operatorname{PhNV}^{2+}(\mathbf{2 3})$ & -0.161 \\
4 & $\mathrm{ACNV}^{2+}(\mathbf{2 6})$ & -0.133 \\
5 & $\mathrm{ACCV}^{2+}(\mathbf{2 7})$ & -0.123 \\
6 & $\mathrm{NCV}^{2+}(\mathbf{2 5})$ & -0.118 \\
\hline
\end{tabular}




\subsubsection{Reactivity with amino thiols}

All five probes were screened for their optical sensing behavior towards the amino thiols Hcy, Cys and GSH. Screening was done at rt, higher concentrations of thiols (1 $\mathrm{mM})$, probe $(4 \mathrm{mM})$ and in various buffers at different $\mathrm{pH}$ conditions (phosphate $\mathrm{pH} 6,7$ and 8; Tris pH 7, 8 and 9; Hepes pH 7 and 8). $\mathrm{PhCV}^{2+}$ (25) produced excellent selectivity towards GSH and this is described in detail in chapter 5. $\operatorname{ACCV}^{2+}(27)$ was selective for Hcy at higher concentrations of the thiols $(1 \mathrm{mM})$. At pH 8 (phosphate buffer, $80 \mathrm{mM}$ ), probe 27 developed an intense color upon reaction with Hcy whereas Cys and GSH samples had the same color as the blank (Figure 4.2). Attempts to lower the thiol concentration below $1 \mathrm{mM}$ resulted in loss of Hcy selectivity and all thiols developed the same color as the blank.



Figure 4.2 Response of $\mathrm{ACCV}^{2+}(\mathbf{2 7})$ towards various amino thiols at $\mathrm{pH} 8 . \mathrm{ACCV}^{2+}(4 \mathrm{mM})$ was added to thiol solutions $(1 \mathrm{mM}$ each) buffered at $\mathrm{pH} 8$ (phosphate buffer $(80 \mathrm{mM})$. From left to right: Blank, Hcy, Cys and GSH. The picture was taken 30 min after mixing solutions at room temperature.

UV-Vis absorption spectroscopy was used to monitor the reaction between the asymmetric probes and biological thiols. As shown in Figure 4.3, upon mixing solutions of $\mathrm{NCV}^{2+}(\mathbf{2 5})$ with amino thiols in phosphate buffer $(80 \mathrm{mM})$ at $\mathrm{pH} 8$, a significant color 
change was observed in the sample containing Cys after 5 min. However, no significant color formation or absorption response was seen in the case of Hcy or GSH samples. Over time, Hcy was found to promote a signal. This is in agreement with our earlier study under reflux conditions where we found that at higher $\mathrm{pH}$, the colorimetric response of symmetric viologen probes towards $\alpha$-carbon radicals from both Hcy and Cys becomes less Hcy selective.

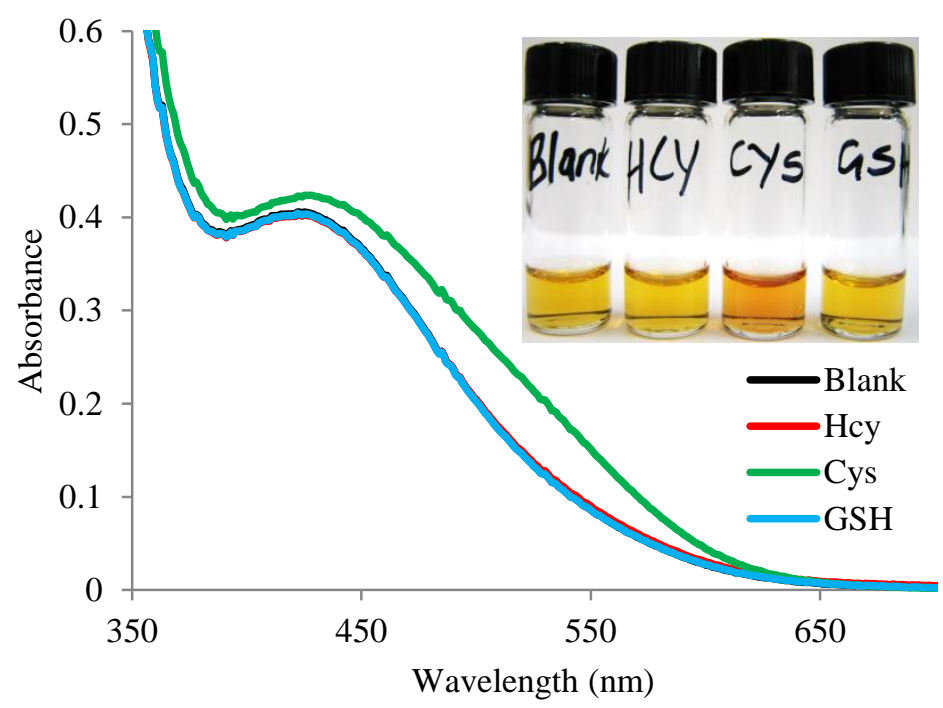

Figure 4.3 Spectral response of $\mathrm{NCV}^{2+}(\mathbf{2 5})$ towards various amino thiols at $\mathrm{pH}$ 8. Absorption spectra of solutions of $\mathrm{NCV}^{2+}(4 \mathrm{mM})$ in thiol solutions $(1 \mathrm{mM})$ buffered at $\mathrm{pH} 8$ (phosphate buffer $(80 \mathrm{mM})$. The inset shows the picture taken at $10 \mathrm{~min}$.

We investigated the reaction of $\mathrm{ACNV}^{2+}(\mathbf{2 6})$ with Hcy, Cys and GSH in $80 \mathrm{mM}$ phosphate buffer $(\mathrm{pH} 8)$ and found that Hcy can be detected selectively under these conditions without interference from related analytes. Through optimization of the reaction, we were able to distinguish Hcy from Cys and GSH at their physiological levels 
in natural media. Upon adding $26(350 \mu \mathrm{M})$ to solutions of Hcy $(15 \mu \mathrm{M})$, Cys $(250 \mu \mathrm{M})$ and GSH $(6 \mu \mathrm{M})$ buffered at $\mathrm{pH} 8$ (phosphate buffer, $80 \mathrm{mM}$ ), a marked color change as well as an intense absorption response was observed for the Hcy solution.

While monitoring the change in absorbance at $547 \mathrm{~nm}$ as a function of time (Figure 4.4) over 2 to $10 \mathrm{~min}$, it was observed that Hcy can be detected selectively without any significant interference from both Cys and GSH. Thereafter, Cys reacts in an opposing fashion to that of Hcy showing a decreased absorbance peak, far below that of both the blank and GSH. The Cys solution peak began to diminish after 10 min, showing a plateau at $25 \mathrm{~min}$. Thus, Hcy can be detected selectively without interference from Cys and GSH at their physiological levels at 10 min (Figure A4, Appendix A).

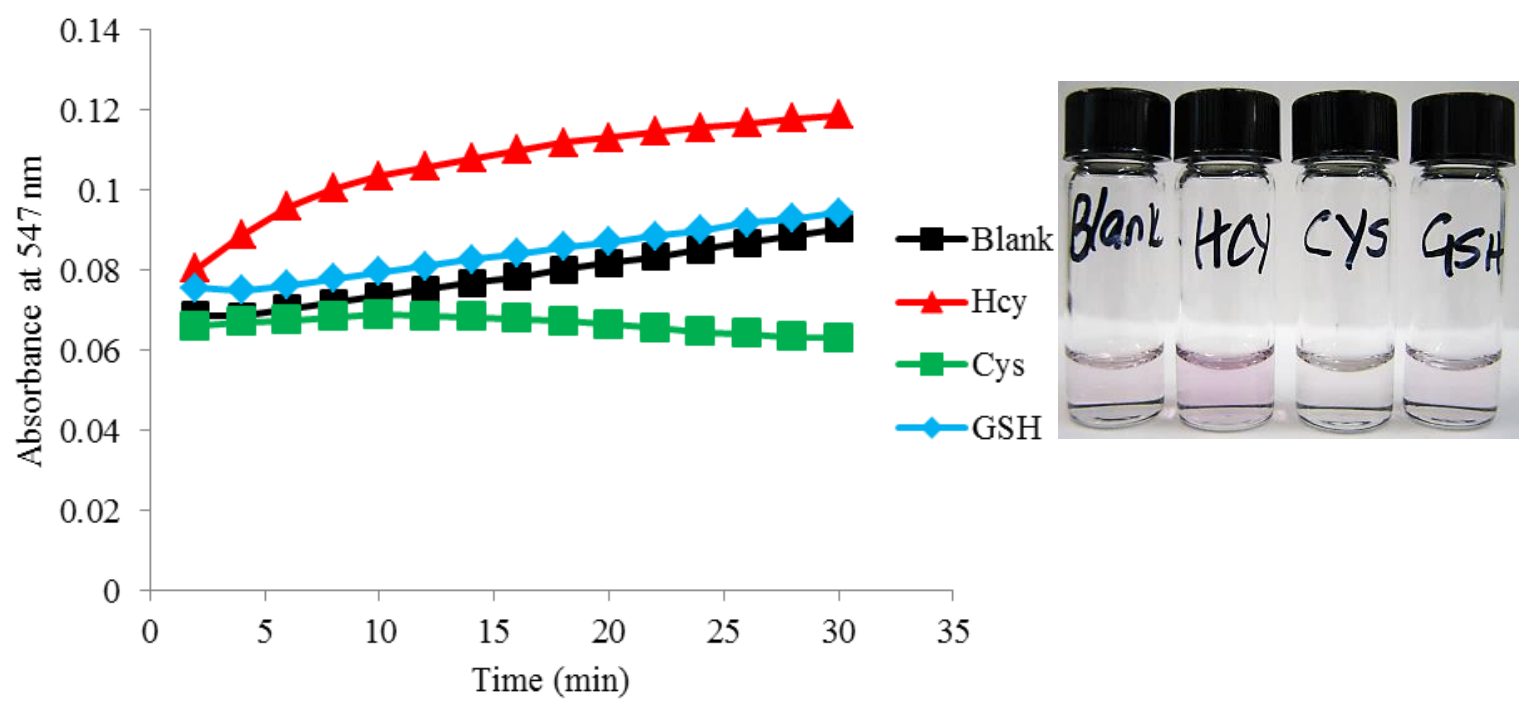

Figure 4.4 Time-dependent spectral response of $\mathrm{ACNV}^{2+}(\mathbf{2 6})$ towards various amino thiols at $\mathrm{pH} 8$. Timedependent absorbance changes of $26(350 \mu \mathrm{M})$ in thiol solutions (15 $\mu \mathrm{M}$ Hcy, $250 \mu \mathrm{M}$ Cys and $6 \mu \mathrm{M} \mathrm{GSH})$ buffered at pH 8 (Phosphate buffer, $80 \mathrm{mM}$ ). The inset shows the picture taken at $30 \mathrm{~min}$. 
The absorption spectra displaying opposing reaction behaviors of $\mathbf{2 6}$ towards Hcy and Cys are shown in Figure 4.5. In the case of Cys the absorbance band at $547 \mathrm{~nm}$ decreased with concomitant growth of the band at $400 \mathrm{~nm}$ over time (Figure 4.5b) whereas for Hcy both bands increased with time (Figure 4.5a). This is the first example, to our knowledge, of a redox-based probe that detects Hcy and Cys simultaneously at typical biological levels found in human plasma.

(a)

(b)

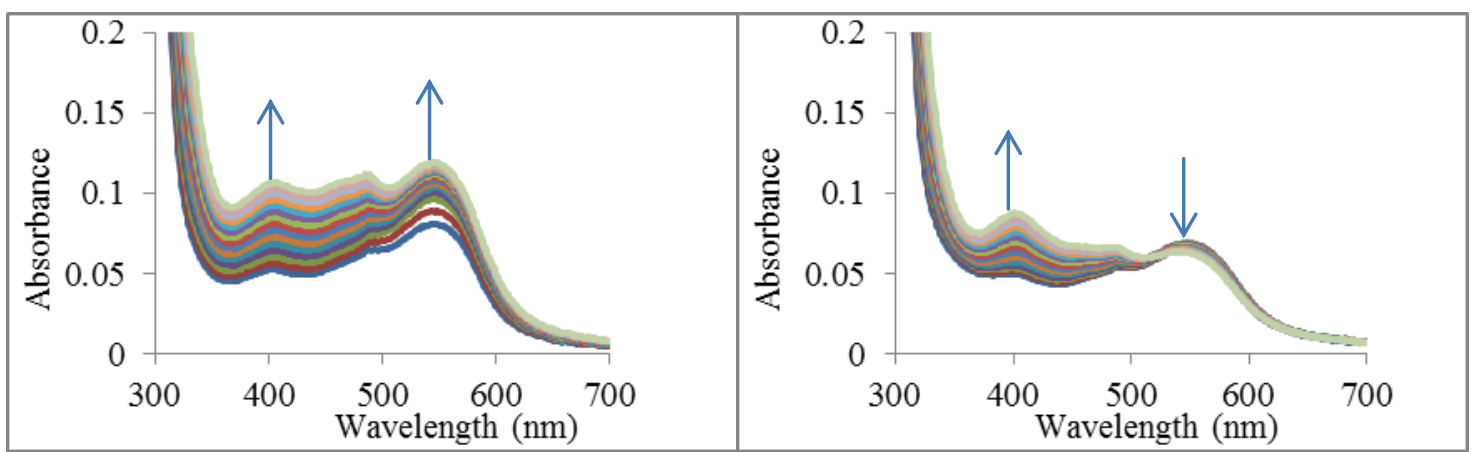

Figure 4.5 Optical sensing behavior of $\mathrm{ACNV}^{2+}$ (26) towards Hcy and Cys at pH 8. (a) Absorption spectra of 26 with Hcy $(15 \mu \mathrm{M})$ over 30 min. (b) Absorption spectra of 26 with Cys $(250 \mu \mathrm{M})$ over 30 min.

From the screening studies, $\mathrm{PhNV}^{2+}(\mathbf{2 3})$ was found to be the most promising probe for detecting Hcy selectively at rt at its physiologically relevant levels. The reaction of $23(2.5 \mathrm{mM})$ with Hcy $(15 \mu \mathrm{M})$, Cys $(250 \mu \mathrm{M})$ and GSH $(6 \mu \mathrm{M})$ at $\mathrm{rt}$, $\mathrm{pH} 8$ was investigated by monitoring the changes in absorbance at $550 \mathrm{~nm}$ as a function of time. Hcy induced a fast response and the absorbance increased rapidly up to about 10 min and then just gradually thereafter (Figure 4.6). As for Cys and GSH solutions, their absorbance changes over 25 min overlapped with the blank demonstrating that this probe is Hcy specific. 
After several hours all the thiols become indistinguishable as the absorbance increase due to Hcy, converges with the absorbance levels from GSH and Cys.

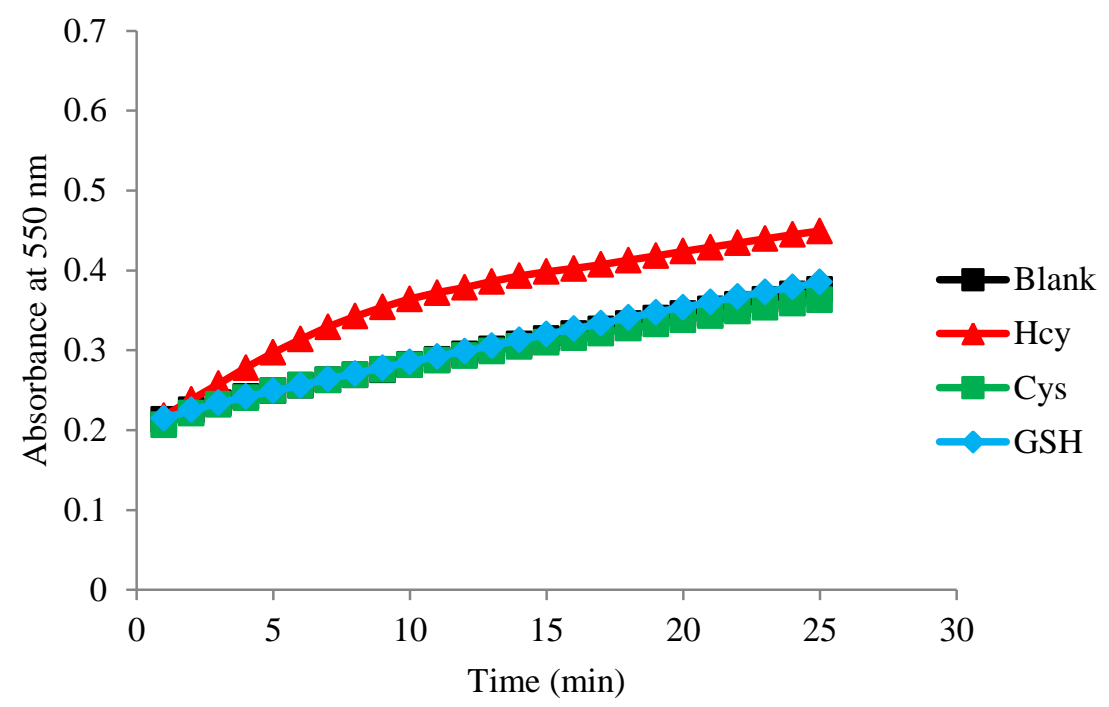

Figure 4.6 Time-dependent spectral response of $\mathrm{PhNV}^{2+}(\mathbf{2 3})$ towards various amino thiols at $\mathrm{pH} 8$. Timedependent absorbance changes of $23(2.5 \mathrm{mM})$ in thiol solutions (15 $\mu \mathrm{M} \mathrm{Hcy,}, 250 \mu \mathrm{M}$ Cys and $6 \mu \mathrm{M} \mathrm{GSH})$ buffered at $\mathrm{pH} 8$ (phosphate buffer, $80 \mathrm{mM}$ ).

Based on these findings, we selected $10 \mathrm{~min}$ as the optimal reaction time for further studies monitoring at $550 \mathrm{~nm}$. The corresponding absorption spectra at $10 \mathrm{~min}$ showed high selectivity for Hcy, however there was a change via emergence of a peak between 400 and $450 \mathrm{~nm}$, showing the blank and Cys increasing relatively slightly (Figure 4.7). 


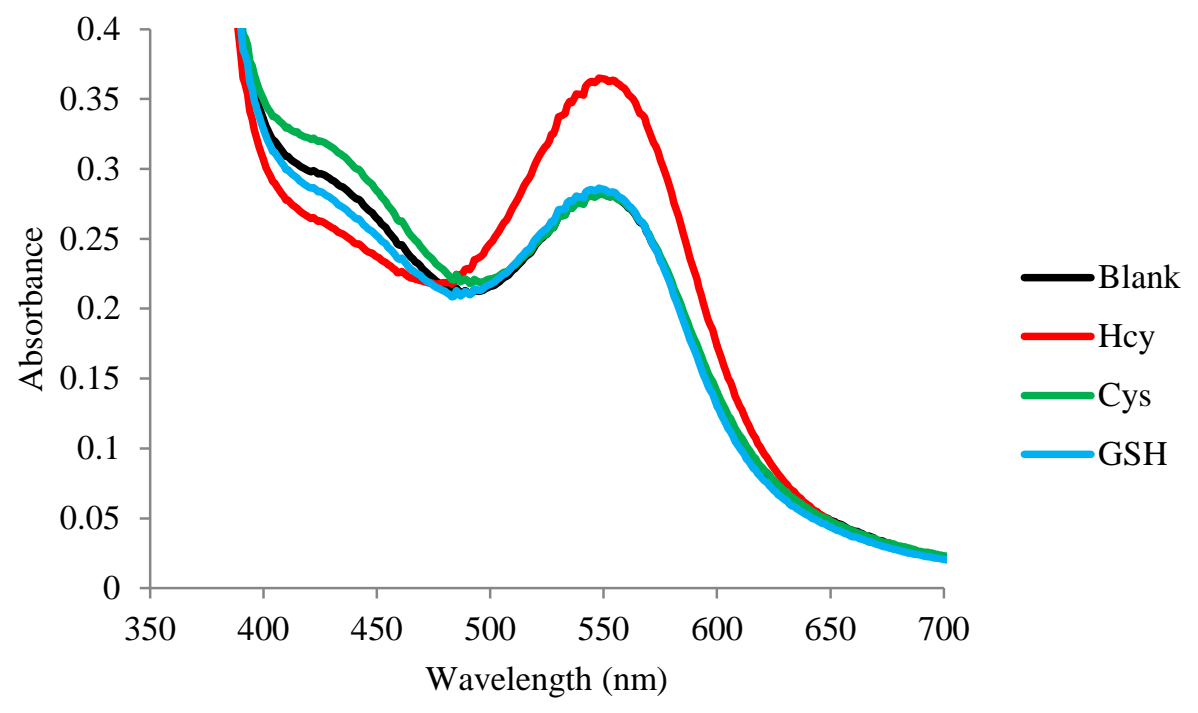

Figure 4.7 Spectral response of $\mathrm{PhNV}^{2+}(\mathbf{2 3})$ towards various amino thiols at $\mathrm{pH}$ 8. Absorption spectra of $23(2.5 \mathrm{mM})$ in thiol solutions (15 $\mu \mathrm{M}$ Hcy, $250 \mu \mathrm{M}$ Cys and $6 \mu \mathrm{M} \mathrm{GSH})$ buffered at pH 8 (phosphate buffer, $80 \mathrm{mM}$ ) after $10 \mathrm{~min}$.

Upon lowering the probe concentration to $2 \mathrm{mM}$, in the presence of thiol solutions (15 $\mu \mathrm{M}$ Hcy, $250 \mu \mathrm{M}$ Cys and $6 \mu \mathrm{M}$ GSH) buffered at pH 8 (phosphate buffer, $80 \mathrm{mM}$ ), 23 developed a strong pink color and generated a significant absorbance response for Hcy without any interference from Cys and GSH (Figure 4.8). Samples containing Cys and GSH developed the same color as the blank and their absorption spectra overlapped. 


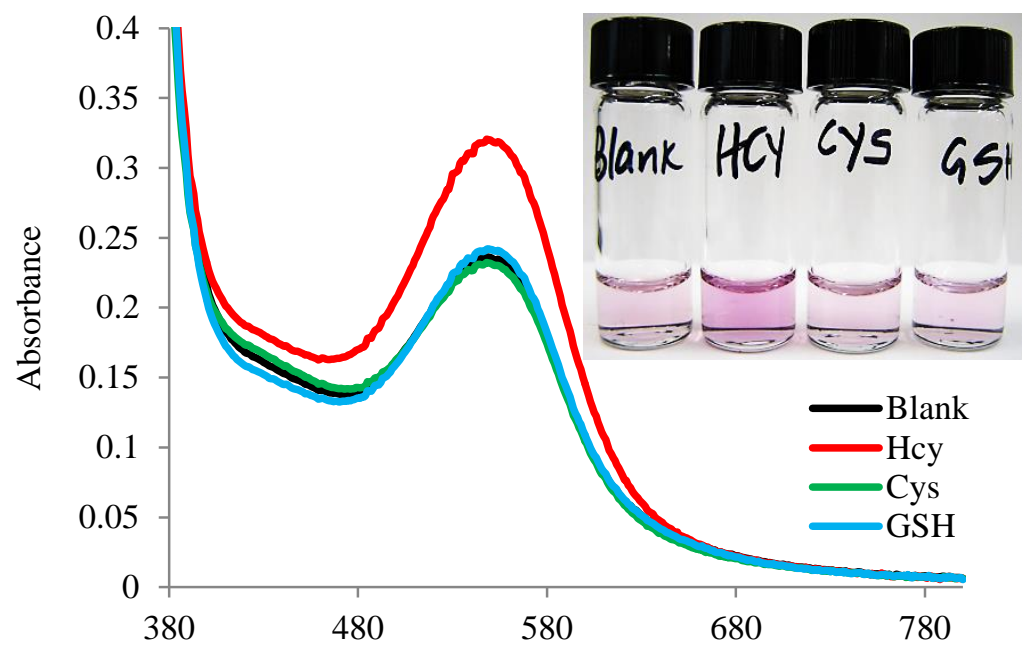

Wavelength (nm)

Figure 4.8 Spectral response of $\mathrm{PhNV}^{2+}(\mathbf{2 3})$ towards various amino thiols at $\mathrm{pH}$ 8. Absorption spectra of solutions of $\mathrm{PhNV}^{2+}(2 \mathrm{mM})$ in thiol solutions $(15 \mu \mathrm{M}$ Hcy, $250 \mu \mathrm{M}$ Cys and $6 \mu \mathrm{M} \mathrm{GSH})$ buffered at $\mathrm{pH} 8$ (phosphate buffer $(80 \mathrm{mM})$. The inset shows the picture taken at $10 \mathrm{~min}$.

The reaction profile of $\mathbf{2 3}$ towards amino thiols further confirmed the specificity of the probe for Hcy as shown by the rapid increase in absorbance relative to that of Cys, GSH and the blank. The absorbance changes for Cys and GSH increased steadily in the same manner as the blank and produced no interference to Hcy over the 15 min period the reaction was monitored (Figure 4.9). 


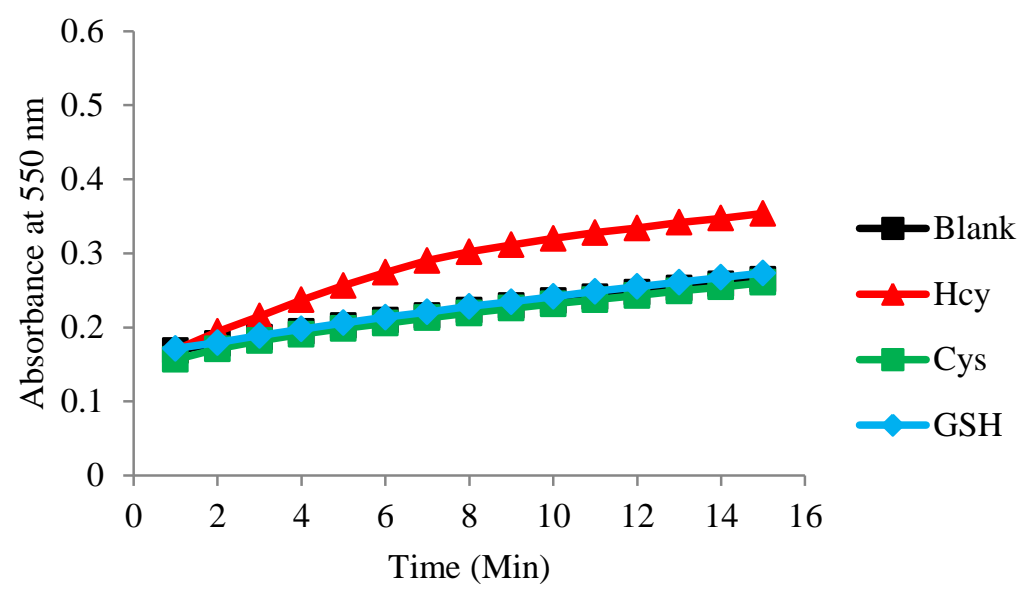

Figure 4.9 Time-dependent spectral response of $\mathrm{PhNV}^{2+}(\mathbf{2 3})$ towards various amino thiols at $\mathrm{pH}$ 8. Timedependent absorbance changes of $\mathbf{2 3}(2 \mathrm{mM})$ in thiol solutions (15 $\mu \mathrm{M} \mathrm{Hcy,} 250 \mu \mathrm{M}$ Cys and $6 \mu \mathrm{M} \mathrm{GSH})$ buffered at $\mathrm{pH} 8$ (phosphate buffer, $80 \mathrm{mM}$ ).

To enable quantitative determination of Hcy, experiments were conducted to evaluate the sensing behavior of $\mathbf{2 3}$ upon increasing the concentration of Hcy. It can be observed in Figure 4.10 that the absorbance changes of $\mathbf{2 3}$ versus Hcy concentration changes at $550 \mathrm{~nm}$ increased linearly proportional to increasing Hcy concentration over a physiologically relevant concentration range $(0-25 \mu \mathrm{M})$ with a very limit of detection of $2.04 \mu \mathrm{M}$. The inset shows the corresponding spectral data. 




Figure 4.10 Concentration dependent responses of $\mathbf{2 3}(2 \mathrm{mM})$ towards Hcy. A Calibration curve for Hcy showing a linear relationship between absorbance at $550 \mathrm{~nm}$ and Hcy concentration $(0-25 \mu \mathrm{M})$ at $\mathrm{pH} 8$ (Phosphate buffer, $80 \mathrm{mM}$ ). The inset shows the absorption spectra of probe $\mathbf{2 3}$ upon addition of increasing Hcy concentrations.

The applicability of $\mathbf{2 3}$ in a complex biological system was evaluated using commercial human plasma. Upon addition of the probe directly to plasma solutions, a very dark pink color developed showing that the probe reacted with proteins. The plasma was then deproteinized and, after addition of $\mathbf{2 3}$ to plasma samples spiked with various amino thiols (15 $\mu \mathrm{M}$ Hcy, $250 \mu \mathrm{M}$ Cys and $6 \mu \mathrm{M} \mathrm{GSH}$ ) at $\mathrm{pH} 8$ (phosphate buffer, 80 $\mathrm{mM})$, only the Hcy spiked sample displayed significant absorption response whereas Cys and GSH spiked solutions responded in the same manner as the blank (Figure 4.11). In deproteinized plasma, the probe functioned similarly as in aqueous conditions; however, a decrease in the absorbance change was observed as a result of matrix effects. The selective response of $\mathbf{2 3}$ to Hcy over Cys and GSH in plasma combined with the 
simplicity of the probe makes it a potential indicator for use in testing Hcy at point of care and in a home setting.



Figure 4.11 Spectral response of $\mathrm{PhNV}^{2+}(\mathbf{2 3})$ towards various spiked thiols in deproteinized human plasma (dep plasma). Absorption spectra of solutions of $\operatorname{PNV}^{2+}(2 \mathrm{mM})$ in human blood plasma $(10 \% \mathrm{v} / \mathrm{v})$ spiked with various thiols (15 $\mu \mathrm{M}$ Hcy, $250 \mu \mathrm{M}$ Cys and $6 \mu \mathrm{M} \mathrm{GSH})$ buffered at $\mathrm{pH} 8$ (phosphate buffer $(80 \mathrm{mM})$. The inset shows the picture taken at $10 \mathrm{~min}$.

\subsubsection{Mechanistic studies}

To evaluate whether a free radical mechanism was responsible for Hcy selectivity using the asymmetric probes at room temperature or not, Electron paramagnetic Resonance (EPR) spectroscopy was performed in the absence and presence of analytes. EPR is spectroscopic technique that is used to detect, distinguish and quantify different reactive species hence, it plays a pivotal role in the investigations of redox-mediated chemical reactions.

As expected, the involvement of radicals in probe reactivity was confirmed by the EPR spectra (Figure 4.12). However, in the absence of Hcy, the probe itself generated 
a radical of high amplitude, an indication of a higher concentration of resonating paramagnetic species in the sample. The reduction in the probe EPR amplitude in the presence of Hcy suggests that Hcy promoted the formation of more radicals which consequently formed radical cation dimers. These radical cation dimers are diamagnetic hence they do not give an EPR signal. ${ }^{89-91}$ What was not clear from EPR studies is whether the mechanism involves hydrogen atom transfer (HAT) which is more favorable for Hcy or some other radical addition process.

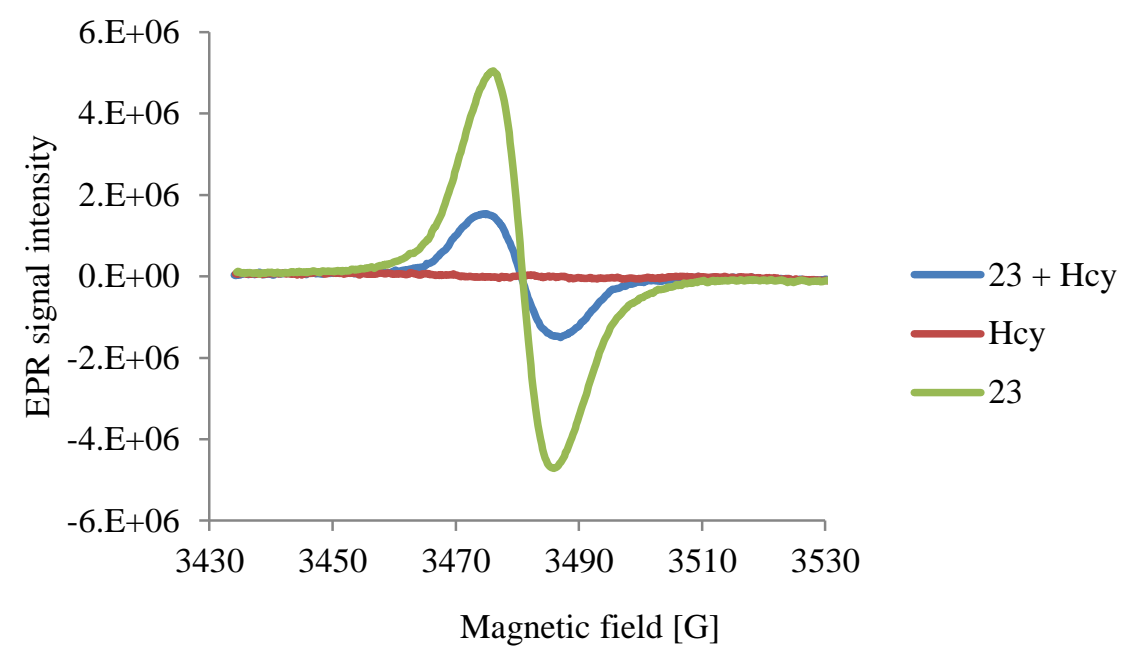

Figure 4.12 EPR spectra of $\mathrm{PhNV}^{2+}(\mathbf{2 3}),(20 \mathrm{mM})$ in the presence and absence of Hcy $(10 \mathrm{mM})$ at $\mathrm{pH} 8$ (80 $\mathrm{mM}$, phosphate buffer).

HAT process occurred selectively for Hcy at neutral $\mathrm{pH}$, but at higher $\mathrm{pH}$ the formation of interfering disulfide radical anions from each thiol renders the method nonselective. However, at $\mathrm{pH} 8$ using the asymmetric probes, we were able to detect Hcy selectively. From our investigations using 2-mercaptoethanol (BME) and 3mercaptopropionic acid (MPA), the involvement of HAT cannot be ruled out. BME and 
MPA are very similar to Hcy in the sense that they possess an - $\mathrm{SH}$ group bearing two methylene groups but they are not capable of producing the $\alpha$-amino carbon centered stabilized radicals. As shown in Figure 4.13, only the sample containing Hcy turned dark red while BME and MPA samples turned yellowish. This suggests that the $\alpha$-amino carbon centered radical is involved in the sensing mechanism.<smiles>N[C@@H](CCS)C(=O)O</smiles>

1<smiles>OCCS</smiles>

BME

28<smiles>O=C(O)CCS</smiles>

MPA

29

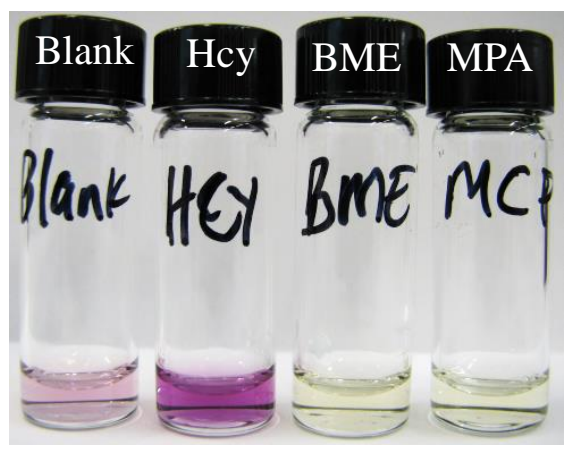

Figure 4.13. Response of $\mathrm{PhNV}^{2+}(\mathbf{2 3})$ towards Hcy, MPA and BME. All solutions were composed of 23 (2 $\mathrm{mM}$ ), analytes (Hcy, MPA and BME, $100 \mu \mathrm{M}$ ) in $80 \mathrm{mM}$ phosphate buffer, $\mathrm{pH}$ 8. Pictures were taken at 30 $\min$.

\subsection{Experimental section}

\subsubsection{Materials and instruments}

All chemicals were purchased from Sigma-Aldrich or Acros Organics and used without further purification. ${ }^{1} \mathrm{H}-\mathrm{NMR}$ and ${ }^{13} \mathrm{C}-\mathrm{NMR}$ spectra were recorded on a Bruker AMX-400 NMR spectrometer, using TMS as an internal standard. Chemical shifts $(\delta)$ are given in ppm relative to DMSO- $d_{6}\left(2.50 \mathrm{ppm},{ }^{1} \mathrm{H}, 39.52{ }^{13} \mathrm{C}\right)$ unless otherwise indicated. 
ESI-HRMS (electrospray ionization - high resolution mass spectrometry) spectra were obtained on a Thermo Electron LTQ Orbitrap hybrid mass spectrometer with a dedicated Accela HPLC system. Electrochemical measurements were performed with a Gamry Reference 600 potentiostat. A three-electrode system was used, consisting of a glassy carbon working electrode, a platinum wire counter electrode and a $\mathrm{Ag} / \mathrm{AgCl}$ reference electrode. Reduction potentials were determined using the half wave reduction potential. EPR studies were performed using a Bruker (Fremont, CA) EMX spectrometer equipped with a high-sensitivity cavity and an Aqua-X sample holder. Spectra were obtained at room temperature. UV-visible spectra were collected on a Cary $50 \mathrm{UV}$-Vis spectrophotometer and $\mathrm{pH}$ measurements were carried out with an Orion 410A pH meter.

\subsubsection{Synthesis of asymmetric viologen probes}

\section{Phenyl-nitrile viologen, $\mathrm{PhNV}^{2+}(\mathbf{2 3})$}

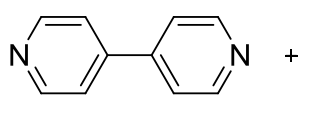

30

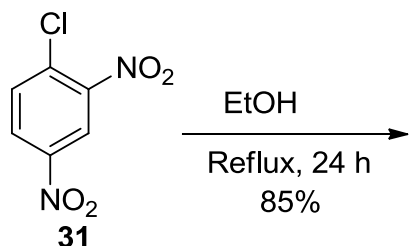

31



33

1. EtOH, r.t, $24 \mathrm{~h}$

2. $\mathrm{Et}_{2} \mathrm{O}, 1 \mathrm{~h}$

3. $\mathrm{H}_{2} \mathrm{O}, 100^{\circ} \mathrm{C}, 24 \mathrm{~h}$

4. $\mathrm{NH}_{4} \mathrm{PF}_{6}, \mathrm{H}_{2} \mathrm{O}$

$66 \%$

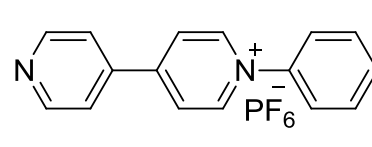

34

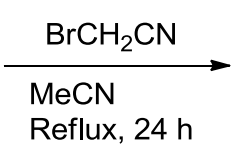

$43 \%$



Figure 4.14 Synthesis of phenyl-nitrile viologen, $\mathbf{P h N V} \mathbf{N}^{\mathbf{2 +}}$ (23) 


\section{1-(2,4-Dinitrophenyl)-4,4'-bipyridin-1-ium chloride $(32)^{92}$}

The synthetic procedure was adapted from work by Coe and co-workers but under anhydrous conditions. ${ }^{92}$ Under Ar, 4,4'-bipyridine 30 (2 g, $\left.12.81 \mathrm{mmol}\right)$ is dissolved in $10 \mathrm{~mL}$ of anhydrous EtOH. To this solution is added 2,4-dinitrochlorobenzene 31 (2.59 g, $12.81 \mathrm{mmol}$ ) in $10 \mathrm{~mL}$ EtOH dropwise and refluxed for $24 \mathrm{~h}$. The mixture is cooled to $\mathrm{rt}$ and added to diethyl ether $(200 \mathrm{~mL})$ with stirring. The resulting golden-brown precipitate is filtered, washed with diethyl ether and dried under vacuum in an argon-saturated glove box to give a light brown solid (3.519 g, 85\%). ${ }^{1} \mathrm{H}$ NMR (400 MHz, DMSO) $\delta 9.66(\mathrm{~d}, J$ $=7.0 \mathrm{~Hz}, 2 \mathrm{H}), 9.14(\mathrm{~d}, J=2.5 \mathrm{~Hz}, 2 \mathrm{H}), 9.05-8.97(\mathrm{~m}, 3 \mathrm{H}), 8.94(\mathrm{dd}, J=4.5,1.6 \mathrm{~Hz}$, 2H), $8.52(\mathrm{~d}, J=8.7 \mathrm{~Hz}, 1 \mathrm{H}), 8.23(\mathrm{dd}, J=4.5,1.7 \mathrm{~Hz}, 2 \mathrm{H})$.

\section{1-(Phenyl)-4,4'-bipyridin-1-ium hexafluorophosphate (34) ${ }^{93,94}$}

Procedures published by Kamogawa et al. and Carballada et al. were modified to synthesize compound 34. Compound $32(1 \mathrm{~g}, 2.79 \mathrm{mmol})$ is dissolved in EtOH $(20 \mathrm{~mL})$ by gentle heating at $>60^{\circ} \mathrm{C}$. A solution of aniline $(33)(1.27 \mathrm{~mL})$ in $10 \mathrm{~mL}$ EtOH is added dropwise and the mixture is stirred at $\mathrm{rt}$ for $24 \mathrm{~h}$. The solvent is removed in vacuo and diethyl ether $(100 \mathrm{~mL})$ added to the residue. This is followed by stirring at $\mathrm{rt}$ for $30 \mathrm{~min}$ and then filtering off the solution. This is repeated two more times until the filtrate is clear and the resulting dark-red residue is heated in $\mathrm{H}_{2} \mathrm{O}(50 \mathrm{~mL})$ for $24 \mathrm{~h}$. An aqueous solution of $1 \mathrm{M} \mathrm{NH}_{4} \mathrm{PF}_{6}$ is added to the filtrate dropwise to give a pale yellow precipitate which is filtered, washed with water and dried under vacuum $(0.693 \mathrm{~g}, 66 \%) .{ }^{1} \mathrm{H}$ NMR 
(400 MHz, DMSO) $\delta 9.53-9.49(\mathrm{~m}, 2 \mathrm{H}), 8.92(\mathrm{~d}, \mathrm{~J}=6.0 \mathrm{~Hz}, 2 \mathrm{H}), 8.81-8.77(\mathrm{~m}, 2 \mathrm{H})$, $8.15(\mathrm{dd}, \mathrm{J}=4.5,1.7 \mathrm{~Hz}, 2 \mathrm{H}), 7.96-7.91(\mathrm{~m}, 2 \mathrm{H}), 7.80-7.76(\mathrm{~m}, 3 \mathrm{H})$.

\section{Phenyl-nitrile viologen, $\mathrm{PhNV}^{2+}(23)$}

Under argon, compound $36(0.200 \mathrm{~g}, 0.529 \mathrm{mmol})$ and bromoacetonitrile $(0.368$ $\mathrm{mL}, 5.29 \mathrm{mmol}$ ) are dissolved in $20 \mathrm{~mL}$ of anhydrous acetonitrile (MeCN). The mixture is heated to reflux for $24 \mathrm{~h}$ and cooled to rt. The resulting precipitate is filtered, washed with fresh $\mathrm{MeCN}$, and dried under vacuum to yield a yellow powder $(0.0985 \mathrm{~g}, 43 \%) .{ }^{1} \mathrm{H}$ NMR (400 MHz, DMSO): $\delta 9.69$ (d, J = 7.0 Hz, 2H), 9.50 (d, J = 7.0 Hz, 2H), 8.95 (dd, J $=9.9,7.0 \mathrm{~Hz}, 4 \mathrm{H}), 7.98-7.94(\mathrm{~m}, 2 \mathrm{H}), 7.82-7.79(\mathrm{~m}, 3 \mathrm{H}), 6.05(\mathrm{~s}, 2 \mathrm{H}) .{ }^{13} \mathrm{C} \mathrm{NMR}(101$ MHz, DMSO) $\delta$ 150.06, 149.02, 146.59, 145.96, 142.23 131.63, 130.26, 127.18, 126.66, 124.81, 114.14 47.80. HR ESI $[\mathrm{M}-\mathrm{H}]^{+}, \mathrm{m} / \mathrm{z}$ : 272.1188; calc for $\mathrm{C}_{18} \mathrm{H}_{14} \mathrm{~N}_{3}{ }^{2+} ; 272.1187$, $\left[\mathrm{M}-\mathrm{C}_{2} \mathrm{H}_{2} \mathrm{~N}\right]^{+}, \mathrm{m} / \mathrm{z}: 233.1079$ calc for $\mathrm{C}_{16} \mathrm{H}_{13} \mathrm{~N}_{2}{ }^{+} 233.1077$.

\section{Phenyl-coumarin viologen, $\mathrm{PhCV}^{2+}(24)$}



34

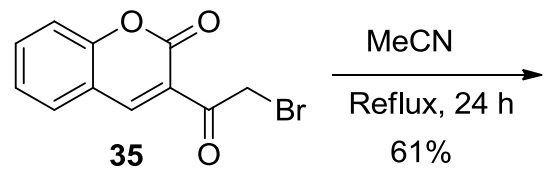

35



Figure 4.15 Synthesis of phenyl-coumarin viologen, $\mathbf{P h C V} \mathbf{C l}^{\mathbf{2 +}}$ (24)

Under argon, compound 34 (0.200 g, $0.529 \mathrm{mmol})$ and 3-(bromoacetyl) coumarin $35(0.706 \mathrm{~g}, 2.64 \mathrm{mmol})$ are dissolved in $20 \mathrm{~mL}$ of anhydrous MeCN. The mixture is 
heated to reflux for $24 \mathrm{~h}$ and cooled to rt. The resulting precipitate is filtered, washed with fresh $\mathrm{MeCN}$, acetone and dried under vacuum to yield a yellow powder $(0.187 \mathrm{~g}$, 61\%). ${ }^{1} \mathrm{H}$ NMR (400 MHz, DMSO): $\delta 9.74(\mathrm{~d}, J=6.5 \mathrm{~Hz}, 2 \mathrm{H}), 9.32(\mathrm{~d}, J=6.4 \mathrm{~Hz}, 2 \mathrm{H})$, 9.04-9.00 (m, 5H), $8.13(\mathrm{~d}, J=7.0 \mathrm{~Hz}, 1 \mathrm{H}), 8.00(\mathrm{~d}, J=3.7 \mathrm{~Hz}, 2 \mathrm{H}), 7.89$ (t, $J=7.8 \mathrm{~Hz}$, 1H), $7.83-7.78(\mathrm{~m}, 3 \mathrm{H}), 7.61(\mathrm{~d}, J=8.4 \mathrm{~Hz}, 1 \mathrm{H}), 7.52(\mathrm{t}, J=7.5 \mathrm{~Hz}, 1 \mathrm{H}), 6.48(\mathrm{~s}, 2 \mathrm{H})$.

${ }^{13} \mathrm{C}$ NMR (101 MHz, DMSO) $\delta 187.59,158.79,154.89,149.60,147.23,145.94,142.15$, $135.98,131.54,130.25,126.53,125.57,124.93,121.12,117.98,116.47,68.95$. HR ESI $[\mathrm{M}-\mathrm{H}]^{+}, \mathrm{m} / \mathrm{z}:$ 419.1396; calc for $\mathrm{C}_{27} \mathrm{H}_{19} \mathrm{~N}_{2} \mathrm{O}_{3}{ }^{2+} 419.1374,[\mathrm{M}+\mathrm{OH}]^{+} \mathrm{m} / \mathrm{z}: 437.1457$ calc for $\mathrm{C}_{27} \mathrm{H}_{20} \mathrm{~N}_{2} \mathrm{O}_{4}{ }^{2+} 437.1478$, [M $\left.-\mathrm{C}_{11} \mathrm{H}_{7} \mathrm{O}_{3}\right]^{+}$, m/z: 233.1079 calc for $\mathrm{C}_{16} \mathrm{H}_{13} \mathrm{~N}_{2}{ }^{+}$233.1066, $[\mathrm{M}+2 \mathrm{H}]^{2+} \mathrm{m} / \mathrm{z}: 420.1474$ calc for $\mathrm{C}_{27} \mathrm{H}_{20} \mathrm{~N}_{2} \mathrm{O}_{3}{ }^{2+} 210.0726$.

\section{Nitrile-Coumarin viologen, $\mathrm{NCV}^{2+}(25)$}

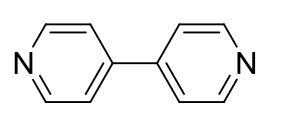

30

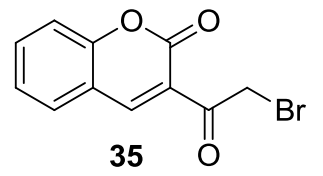



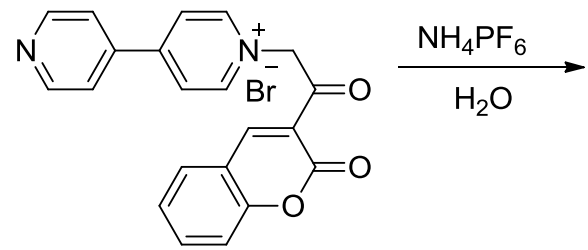

36

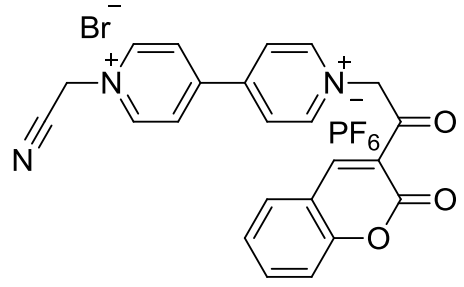

25

37

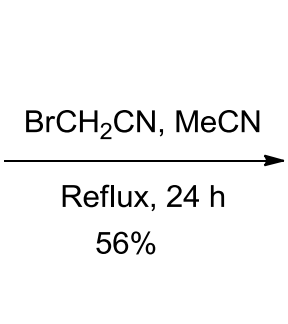

Figure 4.16 Synthesis of Nitrile-coumarin viologen, $\mathbf{N C V}^{2+}(\mathbf{2 5})$. 


\section{1-(2-oxo-2-(2-oxo-2H-chromen-3-yl)ethyl)-[4,4'-bipyridin]-1-ium bromide (36)}

Compound 36 is synthesized following a procedure reported by our group. ${ }^{95} 4,4$ 'bipyridine 30, (1.46 g, $9.36 \mathrm{mmol})$ and 3-(bromoacetyl)coumarin 37 (1 g, $3.74 \mathrm{mmol})$ are dissolved in $40 \mathrm{~mL}$ of acetone and stirred at $\mathrm{rt}$ for $3 \mathrm{~h}$. The precipitate is filtered, washed with acetone and dried under vacuum to give a purple precipitate $(1.279 \mathrm{~g}, 81 \%) .{ }^{1} \mathrm{H}$ NMR (400 MHz, DMSO): $\delta 9.13(\mathrm{~d}, J=6.9 \mathrm{~Hz}, 2 \mathrm{H}), 9.02(\mathrm{~s}, 1 \mathrm{H}), 8.91(\mathrm{dd}, J=4.5,1.6$ $\mathrm{Hz}, 2 \mathrm{H}), 8.77(\mathrm{~d}, J=7.0 \mathrm{~Hz}, 2 \mathrm{H}), 8.15-8.08(\mathrm{~m}, 2 \mathrm{H}), 7.92-7.84(\mathrm{~m}, 1 \mathrm{H}), 7.61(\mathrm{~d}, J=$ $8.4 \mathrm{~Hz}, 1 \mathrm{H}), 7.52(\mathrm{t}, J=7.5 \mathrm{~Hz}, 1 \mathrm{H}), 6.39(\mathrm{~s}, 2 \mathrm{H})$.

\section{Nitrile-coumarin viologen, $\mathrm{NCV}^{2+}(25)$}

The hexafluorophosphate salt (37) was prepared by dissolving the bromide salt (36) in water $(15 \mathrm{~mL})$ followed by dropwise addition of an aqueous solution of $1 \mathrm{M}$ $\mathrm{NH}_{4} \mathrm{PF}_{6}$ until a solid precipitated completely. The light purple precipitate is filtered, washed with $\mathrm{H}_{2} \mathrm{O}$ and dried under vacuum. Under argon atmosphere, compound $\mathbf{3 3}$ $(0.200 \mathrm{~g}, 0.410 \mathrm{mmol})$ and bromoacetonitrile $(0.286 \mathrm{~mL}, 4.10 \mathrm{mmol})$ are dissolved in 20 $\mathrm{mL}$ of anhydrous acetonitrile $(\mathrm{MeCN})$. The mixture is heated to reflux for $5 \mathrm{~h}$ and cooled to rt. The resulting yellow precipitate is filtered, washed with fresh $\mathrm{MeCN}$, recrystallized from EtOH and dried under vacuum to yield a yellow powder $(0.125 \mathrm{~g}, 56 \%) .{ }^{1} \mathrm{H}$ NMR (400 MHz, DMSO): $\delta 9.53(\mathrm{~d}, J=7.0 \mathrm{~Hz}, 2 \mathrm{H}), 9.26(\mathrm{~d}, J=7.0 \mathrm{~Hz}, 2 \mathrm{H}), 9.01(\mathrm{~s}, 1 \mathrm{H})$, $8.90(\mathrm{t}, J=7.0 \mathrm{~Hz}, 4 \mathrm{H}), 8.12(\mathrm{dd}, J=7.9,1.5 \mathrm{~Hz}, 1 \mathrm{H}), 7.92-7.86(\mathrm{~m}, 1 \mathrm{H}), 7.61(\mathrm{~d}, J=$ $8.4 \mathrm{~Hz}, 1 \mathrm{H}), 7.52(\mathrm{dd}, J=11.7,4.3 \mathrm{~Hz}, 1 \mathrm{H}), 6.43(\mathrm{~s}, 2 \mathrm{H}), 6.10(\mathrm{~s}, 2 \mathrm{H}) .{ }^{13} \mathrm{C} \mathrm{NMR}(101$ MHz, DMSO) $\delta 187.83,158.67,154.90,150.43,149.73,147.30,146.50,136.02,131.59$, 
$127.16,126.48,125.56,121.10,117.94,116.40,114.10,69.09,47.76$. HR ESI $[M-H]^{+}$ $\mathrm{m} / \mathrm{z}: 382.1181$ calc for $\mathrm{C}_{23} \mathrm{H}_{16} \mathrm{~N}_{3} \mathrm{O}_{3}{ }^{2+} 382.1175$.

\section{Acetyl-nitrile viologen, $\mathrm{ACNV}^{2+}(26)$}

Compound 26 was synthesized using microwave irradiation by modifying the method used by Lambert and co-workers to synthesize asymmetric viologens. ${ }^{96}$
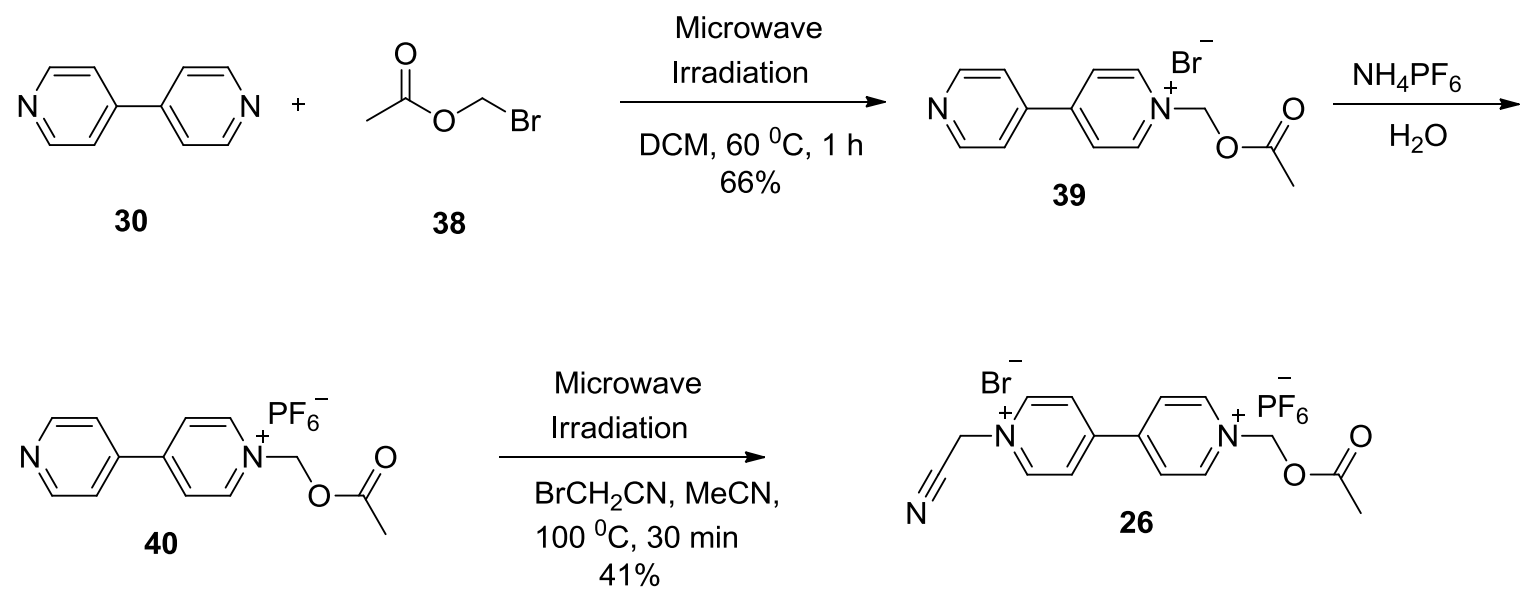

Figure 4.17 Synthesis of Acetyl-nitrile viologen, $\mathbf{A C N V}^{2+}$ (26)

\section{1-(acetoxymethyl)-[4,4'-bipyridin]-1-ium bromide (41)}

4,4'-bipyridine 30, $(0.500 \mathrm{~g}, 3.20 \mathrm{mmol})$ and bromomethylacetate $(0.157 \mathrm{~mL}$, $1.60 \mathrm{mmol}$ ) are dissolved in $4.00 \mathrm{~mL}$ of anhydrous DCM in a sealed microwave vial equipped with a stir bar. The mixture is irradiated on a Biotage Initiator at $60{ }^{\circ} \mathrm{C}$ for $1 \mathrm{~h}$. The vial is cooled to $\mathrm{rt}$ and the resulting precipitate is filtered, washed with fresh DCM and dried under vacuum overnight to give a very pale yellow solid $(0.653 \mathrm{~g}, 66 \%)$. 
${ }^{1} \mathrm{H}$ NMR (400 MHz, DMSO): $\delta 9.34(\mathrm{~d}, J=7.0 \mathrm{~Hz}, 2 \mathrm{H}), 8.89(\mathrm{~d}, J=6.0 \mathrm{~Hz}, 2 \mathrm{H}), 8.69$ $(\mathrm{d}, J=7.0 \mathrm{~Hz}, 2 \mathrm{H}), 8.05(\mathrm{dd}, J=4.5,1.7 \mathrm{~Hz}, 2 \mathrm{H}), 6.45(\mathrm{~s}, 2 \mathrm{H}), 2.17$ (s, 3H). ${ }^{13} \mathrm{C} \mathrm{NMR}$ (101 MHz, DMSO) $\delta$ 169.72, 154.57, 151.03, 145.67, 140.67, 125.32, 122.03, 78.85, 20.57. HR ESI [M-H] $]^{+}, \mathrm{m} / \mathrm{z}: 229.0977$; calc for $\mathrm{C}_{13} \mathrm{H}_{13} \mathrm{~N}_{2} \mathrm{O}_{2}{ }^{+} 229.0969$.

\section{Acetyl-nitrile viologen, $\mathrm{ACNV}^{2+}(26)$}

The hexafluorophosphate salt (40) was prepared following the procedure used for compound 37 above. Compound $40(0.100 \mathrm{~g}, 0.268 \mathrm{mmol})$ and bromoacetonitrile $(0.186$ $\mathrm{mL}, 2.68 \mathrm{mmol}$ ) are dissolved in $4.00 \mathrm{~mL}$ of anhydrous $\mathrm{MeCN}$ in a sealed microwave vial equipped with a stir bar. The mixture is irradiated on a Biotage Initiator at $100^{\circ} \mathrm{C}$ for $30 \mathrm{~min}$. The vial is cooled to rt and the resulting precipitate filtered, washed with fresh DCM and dried under vacuum overnight to give a green-yellowish solid $(0.047 \mathrm{~g}, 41 \%)$. ${ }^{1} \mathrm{H}$ NMR (400 MHz, DMSO): $\delta 9.57-9.46(\mathrm{~m}, 4 \mathrm{H}), 8.86(\mathrm{dd}, J=14.3,6.6 \mathrm{~Hz}, 4 \mathrm{H}), 6.51$ (s, 2H), 6.09 (s, 2H), 2.18 (s, 3H). ${ }^{13} \mathrm{C}$ NMR (101 MHz, DMSO) $\delta$ 169.48, 150.32, 146.31, 127.01, 114.03, 79.31, 47.71, 20.56. HR ESI $[\mathrm{M}-\mathrm{H}]^{+}, \mathrm{m} / \mathrm{z}: 268.1086$ calc for $\mathrm{C}_{15} \mathrm{H}_{14} \mathrm{~N}_{3} \mathrm{O}_{2}{ }^{2+}$ 268.1077, [M $\left.-\mathrm{C}_{2} \mathrm{H}_{2} \mathrm{~N}\right]^{+}, m / z:$ 229.0977; calc for $\mathrm{C}_{13} \mathrm{H}_{13} \mathrm{~N}_{2} \mathrm{O}_{2}{ }^{+}$229.0969, $\left[\mathrm{M}+\mathrm{CH}_{3}\right]^{+}, m / z: 283.1321$ calc for 283.1128 


\section{Acetyl-Coumarin viologen, $\mathrm{ACCV}^{2+}(27)$}

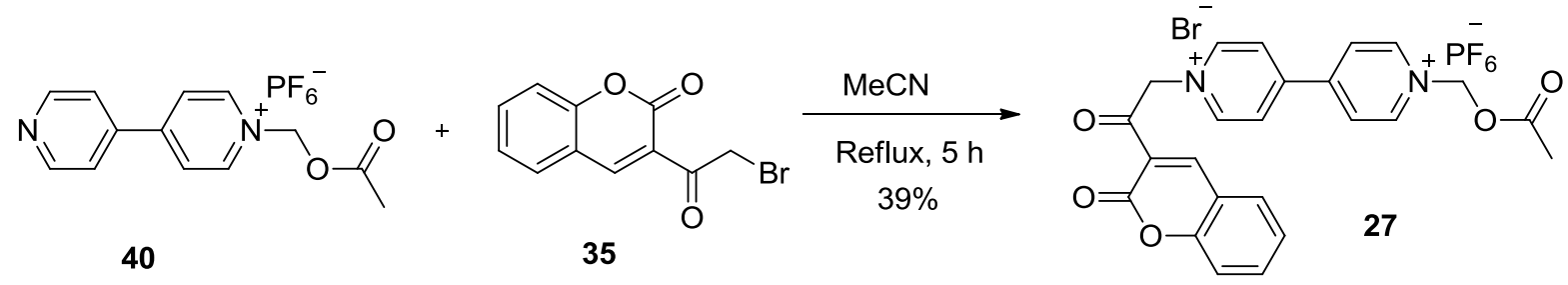

Figure 4.18 Synthesis of Acetyl-coumarin viologen, $\mathbf{A} \mathbf{C C V}^{2+}(\mathbf{2 7})$.

Under Ar, 3-(bromoacetyl) coumarin 35 (0.358 g, $1.34 \mathrm{mmol})$ was dissolved in 15 $\mathrm{mL}$ of anhydrous $\mathrm{MeCN}$ and heated to reflux. A solution of compound $\mathbf{4 0}(0.100 \mathrm{~g}, 0.268$ mmol) in $10 \mathrm{~mL}$ anhydrous $\mathrm{MeCN}$ is added dropwise and the mixture is refluxed for $5 \mathrm{~h}$. After cooling to rt, the resulting precipitate is filtered, washed with fresh $\mathrm{MeCN}$ and dried under vacuum to yield an orange powder (0.0618 g, 39\%). ${ }^{1} \mathrm{H}$ NMR (400 MHz, DMSO): $\delta 9.55(\mathrm{~d}, J=6.9 \mathrm{~Hz}, 2 \mathrm{H}), 9.29(\mathrm{~d}, J=6.9 \mathrm{~Hz}, 2 \mathrm{H}), 9.02(\mathrm{~s}, 1 \mathrm{H}), 8.92(\mathrm{t}, J=7.0 \mathrm{~Hz}, 4 \mathrm{H})$, $8.12(\mathrm{dd}, J=7.8,1.4 \mathrm{~Hz}, 1 \mathrm{H}), 7.92-7.85(\mathrm{~m}, 1 \mathrm{H}), 7.61(\mathrm{~d}, J=8.4 \mathrm{~Hz}, 1 \mathrm{H}), 7.52(\mathrm{dd}, J=$ 11.6, 4.2 Hz, 1H), 6.54 (s, 2H), 6.46 (s, 2H), 2.19 (s, 3H). ${ }^{13} \mathrm{C}$ NMR (101 MHz, DMSO) $\delta$ $187.74,169.56,158.74,154.72,150.99,149.81,149.08,147.20,146.03,136.14,131.67$, 126.72, 125.56, 121.09, 117.76, 116.35, 79.18, 69.05, 20.52. HR ESI $[\mathrm{M}-\mathrm{H}]^{+}, m / z$ : 415.1294 calc for $\mathrm{C}_{24} \mathrm{H}_{19} \mathrm{~N}_{2} \mathrm{O}_{5}{ }^{2+} 415.1279,[\mathrm{M}+\mathrm{OH}]^{+}, \quad m / z: 433.1389$ calc for $\mathrm{C}_{24} \mathrm{H}_{21} \mathrm{~N}_{2} \mathrm{O}_{6}{ }^{2+}$ 433.1384, [M- $\left.\mathrm{C}_{3} \mathrm{H}_{6} \mathrm{O}_{2}\right]^{+}, \mathrm{m} / z:$ 343.1077; calc for $\mathrm{C}_{21} \mathrm{H}_{15} \mathrm{~N}_{2} \mathrm{O}_{3}{ }^{+}$341.1073, $\left[\mathrm{M}-\mathrm{C}_{10} \mathrm{H}_{6} \mathrm{O}_{3}\right]^{+}, m / z: 243.1134$; calc for $\mathrm{C}_{14} \mathrm{H}_{15} \mathrm{~N}_{2} \mathrm{O}_{2}{ }^{+} 243.1124$. 


\subsubsection{Cyclic voltammetry}

Glassy carbon working electrodes were polished with $0.05 \mu \mathrm{m}$ alumina Struers OP-Alumina suspension on a Buehler microcloth, followed by sonication in deionized $\mathrm{H}_{2} \mathrm{O}$. All electrochemical cyclic voltammograms used a Pt flag counter electrode $\left(1 \mathrm{~cm}^{2}\right)$ and a $\mathrm{Ag} / \mathrm{AgCl}$ reference electrode. The electrode was immersed in an aqueous solution a viologen $(4 \mathrm{mM})$ buffered at $\mathrm{pH} 8$ and cycled at a rate of 10,50 or $100 \mathrm{mV} / \mathrm{s}$. All experiments were performed at ambient temperature.

4.3.4 General procedure for the UV/Vis absorption responses of asymmetric viologen probes towards thiols.

The probe was added to solutions of thiols in buffer at $\mathrm{pH} 8.0$ (phosphate buffer, $80 \mathrm{mM}$ ) and vortexed for about 5 seconds to ensure thorough mixing. The solution was then pipetted into a cuvette for UV-Vis spectra collection. In the case of deproteinized plasma, its proportion was $10 \%$ of the total volume after being mixed with buffer and probe solutions. Deproteinized plasma was obtained by reconstituting lyophilized commercial human plasma with Milli- ${ }^{\circledR}$ grade water to $1 / 3$ of the reconstitution volume. Proteins were then precipitated using $\mathrm{MeCN}$ (two thirds of the reconstitution volume) followed by centrifugation at 4,000 rpm for $30 \mathrm{~min}$. The supernatant liquid was decanted, lyophilized for $12 \mathrm{~h}$ and stored at $4{ }^{\circ} \mathrm{C}$ for further use. 


\subsection{Conclusion}

We have developed novel redox-based asymmetric viologen probes that can selectively detect Hcy, Cys and GSH without any interference from each other. PhNV $\mathbf{2}^{\mathbf{}+}$ (23) and $\mathrm{ACNV}^{2+}$ (26) have been proved to be sensitive probes that can detect Hcy selectively at relevant plasma concentrations, which are significantly lower compared to the concentration of competing analytes such as Cys. Additionally, a linear calibration curve obtained for $\mathbf{2 3}$ with increasing Hcy concentration over a physiologically relevant range $(0-25 \mu \mathrm{M})$ with a very low L.O.D of $2.04 \mu \mathrm{M}$ demonstrating a high potential for quantitation of total Hcy in complex biological samples. 


\section{CHAPTER 5: SELECTIVE DETECTION OF GSH USING OPTICAL PROBES}

\subsection{Significance of glutathione to public health}

GSH plays a significant role in the detoxification of free radicals and reactive oxygen species (ROS) thus maintaining and regulating the redox status of cells. ${ }^{97,98}$ Diminished levels of GSH which manifest largely through an increased susceptibility to oxidative stress are characteristic of chronic diseases. Chronic diseases are characterized by long duration and relatively slow progression and include heart disease, stroke, cancer, chronic respiratory diseases and diabetes. They are the leading cause of mortality in the world, accounting for approximately $63 \%$ of all deaths. ${ }^{99}$

Results published in 1999 by Beutler E. et al., revealed a decrease in erythrocyte GSH content in patients affected by $\gamma$-glutamylcysteine synthetase deficiency and hemolytic anemia. ${ }^{100}$ Recently, Atkuri and co-workers reported intracellular reduced glutathione (GSH) deficiency in mitochondrial disorders and organic acidemias. ${ }^{101}$ Mitochondrial disorders can cause organ failure, seizures, stroke-like episodes and premature death. Depleted intracellular GSH levels were also reported in various neurodegenerative disorders such as Alzheimer's and Parkinson's disease. ${ }^{102,103}$ Oxidative stress is the major proposed pathogenic mechanism for neurodegenerative disorders in which mitochondrial defects have been reported. GSH monitoring is a promising new means to diagnose mitochondrial disorders and in clinical trials to monitor autism by indexing the imbalance of oxidative and anti-oxidative stress systems in 
autistic patients. ${ }^{101}$ Mancuso et al. reinforced the same findings that oxidative stress biomarkers which include GSH may be useful in the detection of redox imbalance in mitochondrial diseases and provision of non-invasive tools for monitoring disease status. ${ }^{104}$ Whole blood GSH measurements have also been used to monitor the responses of patients to glutamine (GLN) therapy following open heart surgery. ${ }^{105}$

Conversely, elevated GSH levels generally increase antioxidant capacity and resistance to oxidative stress, and this is observed in many types of cancer cells. ${ }^{98}$ Higher levels of GSH have been reported in a number of different human cancer tissues including, bone marrow, breast, colon and lungs. ${ }^{106-108}$ While GSH is important in the detoxification of carcinogens, its elevated state in cancerous cells and solid tumors makes them more resistant to chemo- and radiotherapy. ${ }^{109}$

\subsection{Current methods for GSH detection}

Current diagnostic methods require a separation step (chromatographic ${ }^{110}$ ), fragile materials (that need skilled handling and specialized storage) and intensive sample processing. ${ }^{111,112}$ Various useful innovative indicators and probes for GSH detection have been recently reported. However, many of them have no specificity for GSH over other structurally similar, competing thiols. ${ }^{113-116}$ Many reports for instance, claim GSH selectivity via fluorescent indicators, however, close scrutiny often reveals that the stated selectivity suffices only in specific applications such as cell imaging, and that Cys, Hcy and other sulfhydril-containing species interfere with the purported GSH indicators. ${ }^{113-116}$ 
Lim et al., reported an activated coumarin-3-phenyl enone fluorescent probe, $\mathbf{4 1}$ which responded selectively and ratiometrically to GSH and Cys over other natural amino acids through the Michael addition reaction. ${ }^{114}$ However, their results showed a significant response from Cys although the GSH response was greater. Additionaly, Hcy another structurally related analyte which could potentially interfere with the system was not investigated using this probe. Since the mechanism involves a Michael addition reaction of the probe to the thiol group, this probe will react with all thiols making it nonspecific for GSH only. Higher concentrations of GSH of $20 \mathrm{mM}$ were used which do not reflect the physiological levels in both plasma and blood cells.

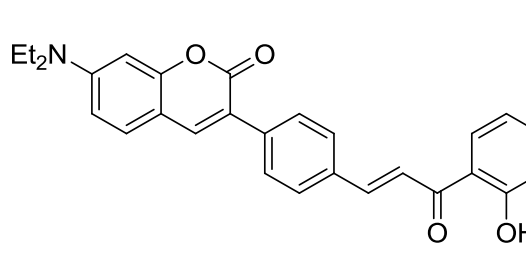

41



42

Figure 5.1. Michael addition reaction of $\mathbf{4 1}$ with thiols. ${ }^{114}$

Another non-specific thiol probe, 1,3,5,7-tetramethyl-8-phenyl-(2-maleimide)difluoroboradiaza-s-indacene, (TMPAB-o-M) was shown to respond to $N$-acetylcysteine and used for labeling BSA in SDS-PAGE. ${ }^{117}$ Subsequently, the same probe was applied in the selective spectrofluorometric determination of glutathione in clinical and biological samples. ${ }^{118}$ Due to the probe's lack of specificity, complex sample preparation and a separation step were needed to help detect GSH selectively. 
There are very few indicators that achieve good selectivity for GSH over other biological thiols. However, some of them have only been applied in cells where GSH concentrations are higher (1-2 $\mathrm{mM})$ but have not been applied in plasma where where its concentrations are very low (1-6 $\mu \mathrm{M})$ compared to those of Cys (135-300 $\mu \mathrm{M})$ and Hcy $(5-15 \mu \mathrm{M}) .{ }^{6,7,10,16}$ Examples include a monochlorinated BODIPY-based ratiometric fluorescent sensor developed by Niu and co-workers, ${ }^{116}$ a bis-spiropyran ligand developed for cellular imaging, ${ }^{119}$ a quantum-dot-based off-on fluorescent probe ${ }^{120}$ and cyanine-based fluorescent probes recently reported by Yin et al. ${ }^{121}$ Our group also reported a dual emission fluorescent probe, a rhodol thioester that detects GSH and Cys/Hcy simultaneously. ${ }^{122}$

We had an impressive achievement in developing a simple probe that can detect GSH levels both in plasma and in cells. This highly sensitive and selective probe is acrylate-based where the discrimination of GSH from structurally related analytes is catalyzed by a cationic surfactant, cetyltrimethylammonium bromide (CTAB) micelle. The resorufin-acrylate indicator was successfully applied in deproteinized human blood plasma and will be described in detail in section 5.3. ${ }^{123,124}$ Prior to that, Strongin's group had designed a seminaphthofluorescein (SNF) acrylate-based probe (43) which detected Cys selectively over GSH and Hcy. ${ }^{125}$ The sensing mechanism was based on a tandem conjugate addition of $\mathbf{4 3}$ to the thiol group followed by an intramolecular cyclization reaction to release the free SNF fluorescent dye (Figure 5.2). The conjugate addition reaction to form thio-ethers is non-selective; however, the second step (intramolecular cyclization) differs among the three thiols due to different kinetics. The intramolecular 
cyclization reaction to form a 7-membered ring for Cys is kinetically favored over the formation of 8- and 12-membered rings in the case of Hcy and GSH respectively. Thus Cys selectivity was based on different relative rates of intramolecular cyclization.

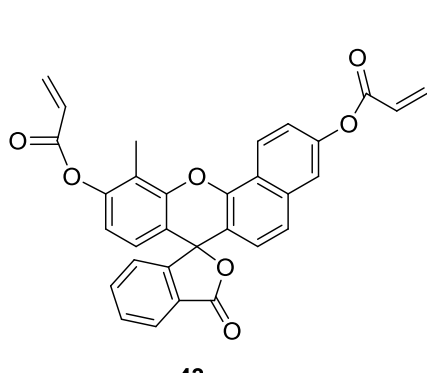

43

Colorless, non-fluorescent


45

Colorful, fluorescent



Hcy, $n=2$

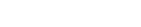

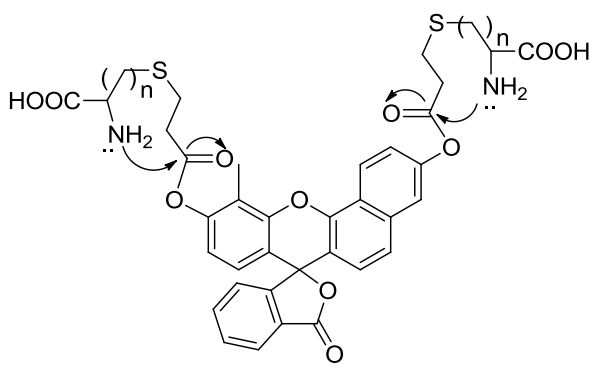

$44 a, n=1$

44b, $n=2$

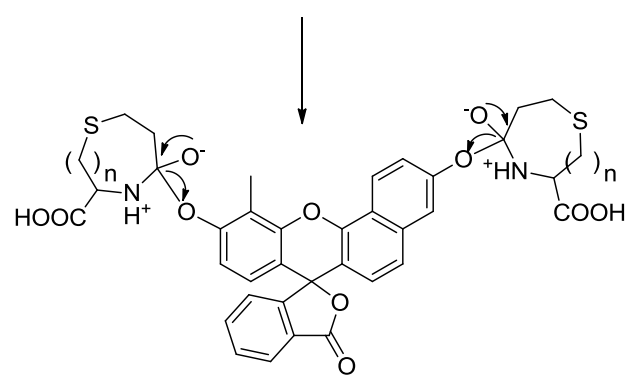

Figure 5.2 Proposed mechanism for discrimination of Cys from Hcy using probe $45{ }^{125}$

To achieve selective detection of GSH over Cys and Hcy, one would need to overcome the formidable challenge of favoring the formation of a larger ring (12membered for GSH) over a 7- and 8-membered ring in the case of Cys and Hcy respectively. Wei and co-workers reported that cetyl-trimethyl-ammonium bromide (CTAB, 46) micelles can catalyze the intramolecular ring closure of larger rings, reversing the expected kinetics based on ring size. ${ }^{126}$ In their study, they measured the 
rates of cyclization of an intramolecular Williamson ether reaction (Figure 5.3) for the formation of 7 - to 20 -membered rings and observed a 500-1500-fold decrease in ring closure on going from a 7-membered ring to a 14-membered ring in homogeneous environments (99\% DMSO, 75\% EtOH) and only a 6-fold decrease in the micellelocalized reaction.

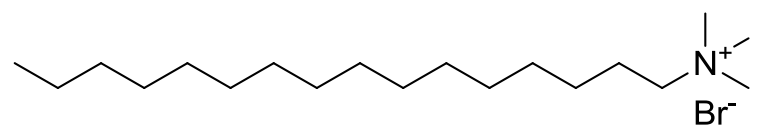

cetyl-trimethyl-ammonium bromide (CTAB)

46



47

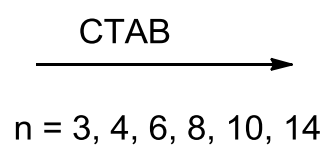

$\mathrm{n}=3,4,6,8,10,14$

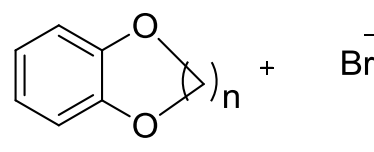

48

Figure 5.3 Intramolecular Williamson ether reaction. ${ }^{126}$

In another study, it was reported that GSH binds the positively charged surface of CTAB micelle through some electrostatic interactions between the carboxylate groups and the cationic CTAB surface. ${ }^{127}$ Based on this, we envisioned that a relatively smaller, flat and more planar dye similar to $\mathrm{PhSeO}_{2} \mathrm{H}$ (Figure 5.4) should fit well within the micelle allowing a good self-assembly of CTAB micelle with the probe through hydrophobic and electrostatic interactions in water bringing it in close proximity to GSH. 
We then proceeded to design a very simple resorufin-acrylate based probe that successfully detected GSH in human plasma.



Figure 5.4 Proposed Model for Selenium-Mediated Micellar. ${ }^{127}$

*Adapted with permission from Langmuir, 2007, Volume 23, pages 1518-1522; Huang, X.; Dong, Z.; Liu, J.; Mao, S.; Xu, J.; Luo, G.; Shen, J. Selenium-Mediated Micellar Catalyst: An efficient Enzyme Model for Glutathione Peroxidase-like Catalysis. Copyright 2007 American Chemical Society.

To rule out the possibility of detecting GSH using acrylate-based probes which are large and 3-dimensional in shape in micelles, we designed a near-infrared (NIR) naphthofluorescein-based dye (49) and investigated its reaction selectivity in the presence of aminothiols. This probe was selective for Cys, thus eliminating any possibilities of achieving GSH selectivity with non-planar compounds. As predicted, the resorufin acrylate probe (50) was successful in detecting GSH in deproteinized human plasma without any interference from related analytes such as Hcy and Cys. While developing 
asymmetric viologen probes specific for Hcy, to our surprise one of them, $\mathrm{PhCV}^{2+}(\mathbf{2 4})$, detected GSH selectively without any interference from Hcy and Cys. This will be described in the next section.

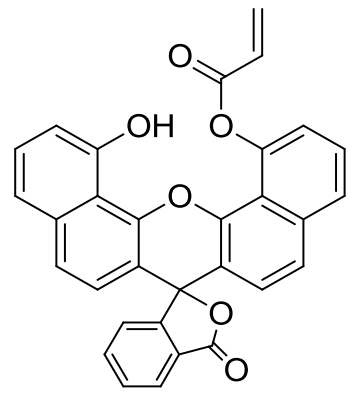

49<smiles>C=CC(=O)Oc1ccc2nc3ccc(=O)cc-3oc2c1</smiles>

50

Figure 5.5 Structures of acrylate-based probes

\subsection{Results and discussion}

\subsubsection{Acrylate-based probes}

Probe 49 showed excellent selectivity towards Cys due to the reported kinetically favored formation of a 7-membered ring in the intramolecular cyclization step over the 8and 12-membered rings for Hcy and GSH respectively. Upon reacting 49 with amino thiols, only the Cys containing sample developed a green color of the free dye whereas Hcy and GSH remained unchanged (Figure 5.6). Additionally, a significant absorbance response was observed for Cys and absolutely no response for related analytes. The concentrations of Cys and GSH used reflect their normal biological concentration in human plasma whereas the $15 \mu \mathrm{M}$ of Hcy was used to represent mild homocysteinemia. These findings indicate that this probe can serve as a selective "off-to-on" dosimeter for 
Cys with potential application in natural media. Moreover, the results confirmed our hypothesis that a relatively smaller, flat and more planar dye and not a large, 3 dimensional dye would fit well within the micelle allowing a good self-assembly of CTAB micelle with the probe for reaction with GSH.

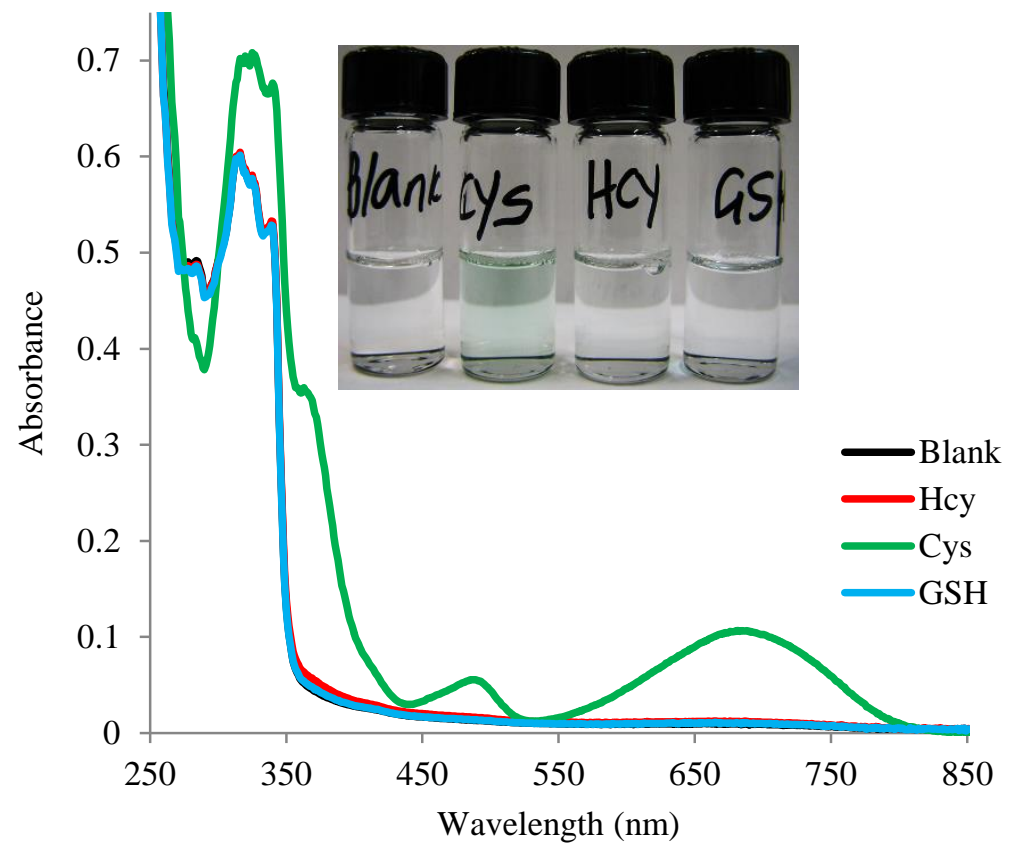

Figure 5.6 CTAB-mediated response of $\mathbf{4 9}$ towards various aminothiols at $\mathrm{pH}$ 7.4. Absorption spectra of solutions of $49(50 \mu \mathrm{M})$ in thiol solutions (15 $\mu \mathrm{M}$ Hcy, $250 \mu \mathrm{M}$ Cys and $6 \mu \mathrm{M}$ GSH) buffered at $\mathrm{pH} 7.4$ (Phosphate buffer $(50 \mathrm{mM})$. The inset shows the picture taken at $10 \mathrm{~min}$.

To this end a resorufin acrylate probe (50) was developed and successfully applied in detecting GSH selectively via my coworkers using a CTAB micelle. ${ }^{123}$ Solutions of GSH, Cys and Hcy ( 2 equiv) with $\mathbf{5 0}$ in $\mathrm{pH} 7.4$ phosphate buffer generated a characteristic strong pink color immediately upon addition of CTAB only in the presence of GSH (Figure 5.7a). Other amino acids, such as Hcy and Cys, did not exhibit significant changes under the same conditions. This interesting feature indicates that 4- 
CTAB system can serve as a selective visual inspection dosimeter for GSH. Corresponding fluorescence increases were also observed (Figure 5.7b). Fluorescence upon reaction with GSH reached a plateau in less than 2 min.

(a)



(b)



Figure 5.7 Surfactant-mediated responses of solutions containing 50-CTAB towards various thiols at $\mathrm{pH}$ 7.4. (a) Absorption spectra of $\mathbf{5 0}(2.5 \mu \mathrm{M})$ upon addition of thiols (2 equiv) in $2.0 \mathrm{mM}$ CTAB media buffered at $\mathrm{pH} 7.4$ (phosphate buffer, $50 \mathrm{mM}$ ). The inset shows color changes of the solution of $4(10 \mu \mathrm{M})$ upon addition of thiols ( 2 equiv) in $2.0 \mathrm{mM} \mathrm{CTAB} \mathrm{media} \mathrm{buffered} \mathrm{at} \mathrm{pH} 7.4$ (phosphate buffer, $50 \mathrm{mM}$ ). The picture was taken immediately upon addition of CTAB; (b) Fluorescence spectra $(\lambda \mathrm{ex}=565 \mathrm{~nm})$ of $\mathbf{5 0}$ $(2.5 \mu \mathrm{M})$ upon addition of thiols (2 equiv) in $2.0 \mathrm{mM} \mathrm{CTAB}$ media buffered at pH 7.4 (phosphate buffer, $50 \mathrm{mM})$. Spectra were taken immediately upon addition of CTAB. The inset shows time-dependent fluorescence changes $(\lambda \mathrm{em}=587 \mathrm{~nm})$ of the same system.

Based on the above results, we proposed a plausible mechanism for the GSH selectivity of the probe-CTAB system that involves the conjugate addition of GSH to $\mathbf{5 0}$ to generate 51, which in turn undergoes an intramolecular cyclization/elimination reaction sequence catalyzed by the $\mathrm{CTAB}$ micelle releasing the free resorufin dye (Figure 5.8 ), resulting in the recovery of the fluorescence. Formation of the 12-membered ring (52) and the free resorufin dye (53) were confirmed by HRMS. 

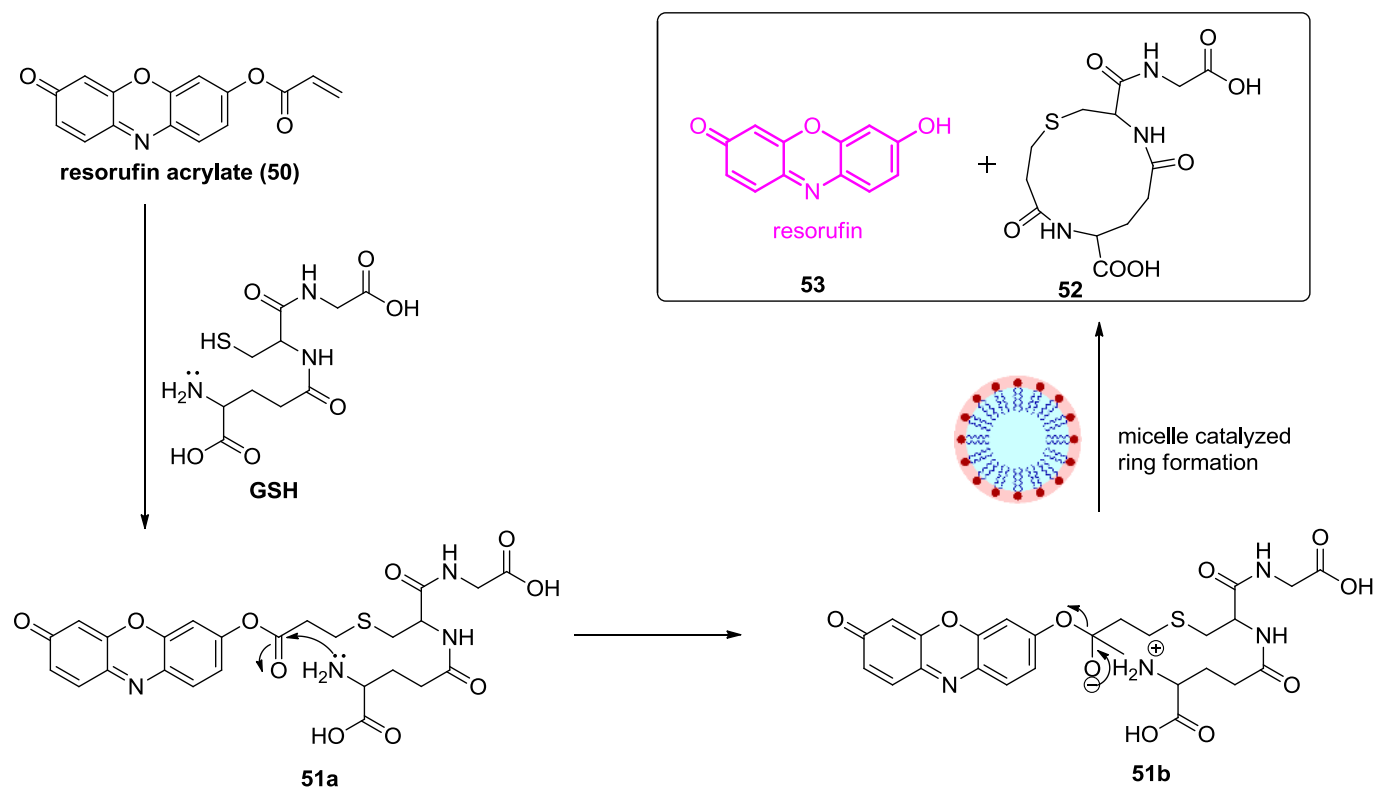

Figure 5.8. Proposed mechanism for the selective detection of GSH in CTAB medium that involves a conjugate addition reaction followed by a micelle-promoted 12-membered ring formation/elimination sequence. ${ }^{123}$

\subsubsection{Improved resorufin-acrylate selectivity towards $\mathrm{GSH}^{124}$}

While the probe-CTAB-GSH signal is high at $\mathrm{pH} 7.4$, it is known that acrylate esters are susceptible to hydrolysis at high $\mathrm{pH}$ and that GSH is also relatively unstable under the same conditions (half-life of 9 hours at $\mathrm{pH} 7.4$ compared to 16 hours at $\mathrm{pH}$ 6.5). ${ }^{128}$ Chemical stability of fluorogenic probes is very important because spontaneous hydrolysis raises background fluorescence and compromises the ability to assess its reactivity with analytes. To address the challenge of probe hydrolysis, my own work involved investigating and optimizing the effect of $\mathrm{pH}$ on the chemical stability of the probe. 
The spectral behavior of the probe-CTAB system was examined over a wide range of $\mathrm{pH}$ values ( $\mathrm{pH} 5.5$ to $\mathrm{pH} 8$ ) and its response to the various thiols was studied at lower $\mathrm{pH}$ values. As expected, the acrylate-based probe $\mathbf{5 0}$ showed the greatest background signal at the higher $\mathrm{pH}$ values investigated. The probe-CTAB system was most stable at lower $\mathrm{pH}$ values (Figure 5.9).



Figure 5.9 Spectral behavior of the probe-CTAB system under different $\mathrm{pH}$ conditions $(50 \mathrm{mM}$ phosphate buffer). Time-dependent absorbance changes of a solution of $\mathbf{5 0}(2.5 \mu \mathrm{M})$ at $580 \mathrm{~nm}$ in CTAB media (2 $\mathrm{mM}$ ) buffered at $\mathrm{pH} 5.5$ to 8 .

We investigated the response of $\mathbf{5 0}$ towards amino thiols at $\mathrm{pH} 6$ and observed that the reaction is less rapid as compared to $\mathrm{pH} 7.4$ (Figure 5.10) and the selectivity towards GSH was further enhanced through the complete elimination of any residual Cys and Hcy response (Figure 5.11). Very little background interference from the hydrolysis of the probe is observed starting at 10 min after mixing. Thus, $\mathrm{pH} 6$ was chosen for further application studies in plasma. 




Figure 5.10 Surfactant mediated response of the probe-CTAB system towards various thiols $(5 \mu \mathrm{M})$ at $\mathrm{pH}$ 6.0. Time-dependent absorbance changes of a solution of $\mathbf{5 0}(2.5 \mu \mathrm{M})$ at $583 \mathrm{~nm}$ in CTAB media $(2 \mathrm{mM})$ buffered at $\mathrm{pH} 6$ (phosphate buffer, $50 \mathrm{mM}$ ).



Figure 5.11 Absorption spectra of $\mathbf{5 0}(2.5 \mu \mathrm{M})$ upon addition of thiols (2 equiv) in $2.0 \mathrm{mM} \mathrm{CTAB}$ media buffered at pH 6 (phosphate buffer, $50 \mathrm{mM}$ ) at $8 \mathrm{~min}$. 
To evaluate the applicability of the probe-CTAB system in complex natural media, fluorescence determination was performed in $10 \%$ deproteinized human plasma. Plasma proteins were precipitated using $\mathrm{MeCN}$ (two thirds of the reconstitution volume) and removed by centrifugation at $4,000 \mathrm{rpm}$ for $30 \mathrm{~min}$. The supernatant liquid was diluted and added to a solution of $\mathbf{5 0}(1 \mu \mathrm{M})$ in phosphate buffer $(\mathrm{pH} 6,50 \mathrm{mM})$ in the presence of $2.0 \mathrm{mM} \mathrm{CTAB}$. The fluorescence response of replicate $(\mathrm{n}=3)$ samples and GSH spiked samples were monitored as above and the GSH content in the plasma sample was determined from the regression equation of a standard calibration curve (Figure 5.12 (a) and (b)). The GSH content of the plasma sample was determined to be $3.24 \pm 0.14$ $\mu \mathrm{M}$, which is well within the reported GSH concentration range for human plasma samples from healthy individuals. ${ }^{129}$

(a)

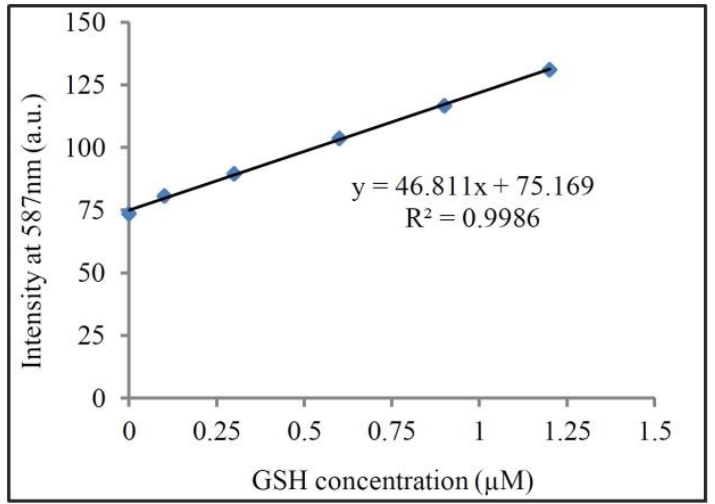

(b)

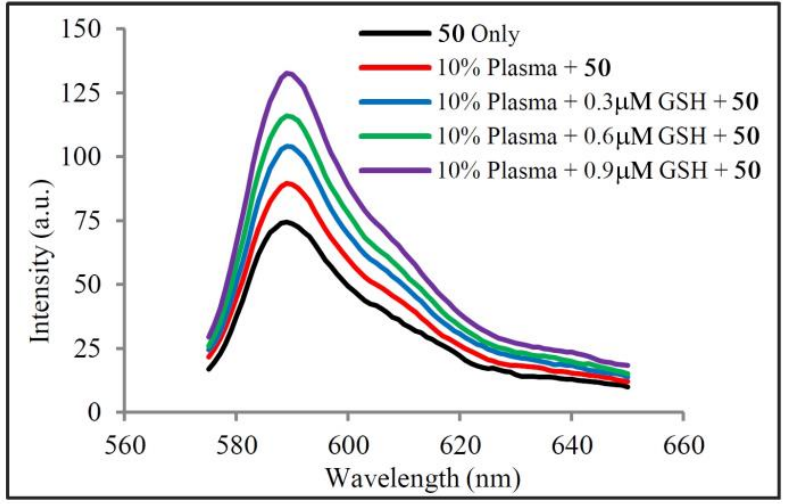

Figure 5.12. Determination of GSH in human plasma using the probe-CTAB system. (a) Calibration curve of $50(1.0 \mu \mathrm{M})$ in $2.0 \mathrm{mM}$ CTAB media buffered at $\mathrm{pH} 6$ (phosphate buffer, $50 \mathrm{mM}$ ) showing a linear relationship between fluorescence intensity $(\lambda \mathrm{ex}=565 \mathrm{~nm}, \lambda \mathrm{em}=587 \mathrm{~nm})$ and GSH concentration $(0.1-$ $1.2 \mu \mathrm{M})$; (b) Fluorescence spectra $(\lambda \mathrm{ex}=565 \mathrm{~nm})$ of $\mathbf{5 0}(1.0 \mu \mathrm{M})$ upon addition of GSH $(0-1.0 \mu \mathrm{M})$ to $10 \%$ deproteinized plasma diluted with $2.0 \mathrm{mM}$ CTAB media buffered at $\mathrm{pH} 6.0$ (phosphate buffer, 50 $\mathrm{mM})$. The calibration curve and emission spectra were collected 8 min after mixing. 
Accuracy assessment of the method was conducted using recovery experiments. ${ }^{130}$ Recoveries of the known spiked amounts of GSH were between $99.2 \%$ and $102.3 \%$ with a generally satisfactory precision (Table 5.1). These results revealed the potential applicability and reliability of using $\mathbf{5 0}$ in quantitative detection of GSH in human plasma.

Table 5.1. Determination of GSH content in 10\% deproteinized human plasma samples.

\begin{tabular}{lcccc}
\hline Sample & $\begin{array}{c}\text { GSH Spiked } \\
(\mu \mathrm{M})\end{array}$ & $\begin{array}{c}\text { GSH Measured } \\
(\mu \mathrm{M})\end{array}$ & $\begin{array}{c}\text { Recovery } \\
(\%)\end{array}$ & $\begin{array}{c}\text { RSD } \\
(\%)\end{array}$ \\
\hline $10 \%$ Plasma & 0 & 0.324 & - & 4.42 \\
$10 \%$ Plasma + GSH & 0.3 & 0.631 & 102.3 & 4.90 \\
$10 \%$ Plasma + GSH & 0.6 & 0.919 & 99.2 & 4.24 \\
$10 \%$ Plasma + GSH & 0.9 & 1.224 & 100.0 & 5.88 \\
\hline
\end{tabular}

\subsubsection{Asymmetric viologen-based probe}

The response of $\mathrm{PhCV}^{2+}(\mathbf{2 4})$ towards various aminothiols was examined at $\mathrm{pH} 9$ (Tris buffer, $0.5 \mathrm{M}$ ). Initial investigation of $\mathbf{2 4}$ at a concentration of $1 \mathrm{mM}$ in thiols solutions (1 $\mathrm{mM})$ showed no selectivity. The reaction was optimized by lowering probe/thiols concentration and surprisingly at $500 \mu \mathrm{M}$ probe concentration and $500 \mu \mathrm{M}$, a useful absorption response was observed in the presence of GSH (Figure A8, Appendix A). A minimal response was promoted by the presence of Hcy and Cys. Further optimization via lowering the thiol concentration to $250 \mu \mathrm{M}$ resulted in the complete elimination of residual responses from Hcy and Cys, thereby affording excellent GSH selectivity (Figure 5.13). Attempts to lower thiol concentration to physiological levels (15 $\mu \mathrm{M}$ Hcy, $250 \mu \mathrm{M}$ Cys and $6 \mu \mathrm{M}$ GSH) led to loss of selectivity. Hence, this probe cannot 
be applied to determine GSH at relevant plasma levels. However, the probe is potentially of high utility for detecting GSH in blood where levels are higher (1-2 mM). ${ }^{1,4}$

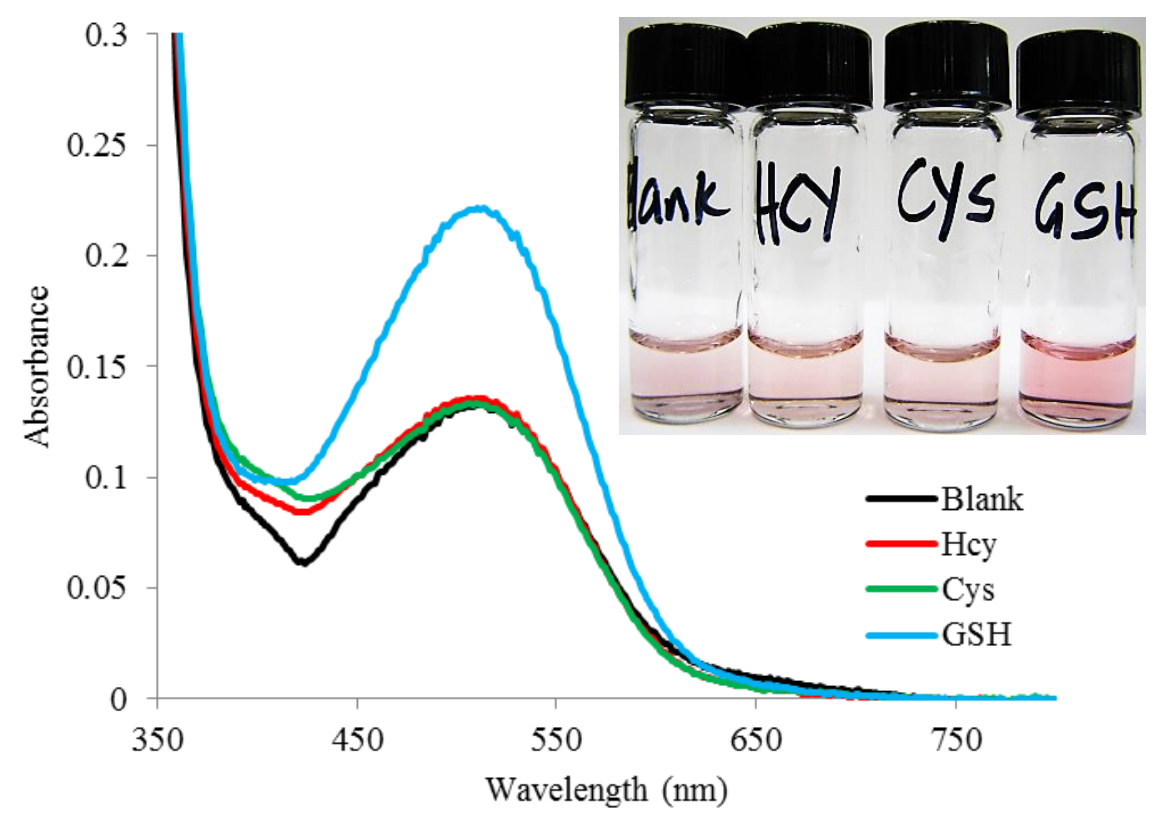

Figure 5.13 Spectral response of $\mathrm{PhCV}^{2+}(\mathbf{2 4})$ towards various amino thiols at $\mathrm{pH}$ 9. Absorption spectra of solutions of $\mathrm{PhCV}^{2+}(500 \mu \mathrm{M})$ in thiol solutions $(250 \mu \mathrm{M})$ buffered at $\mathrm{pH} 9$ (Tris buffer $(200 \mathrm{mM})$. The inset shows the picture taken at $25 \mathrm{~min}$.

Concentration-dependent studies were performed, and absorbance changes at 510 $\mathrm{nm}$ were found to be linearly proportional to the concentrations of GSH $(0-500 \mu \mathrm{M})$. This suggested a potential application towards quantitative determination of GSH (Figure 5.14). 


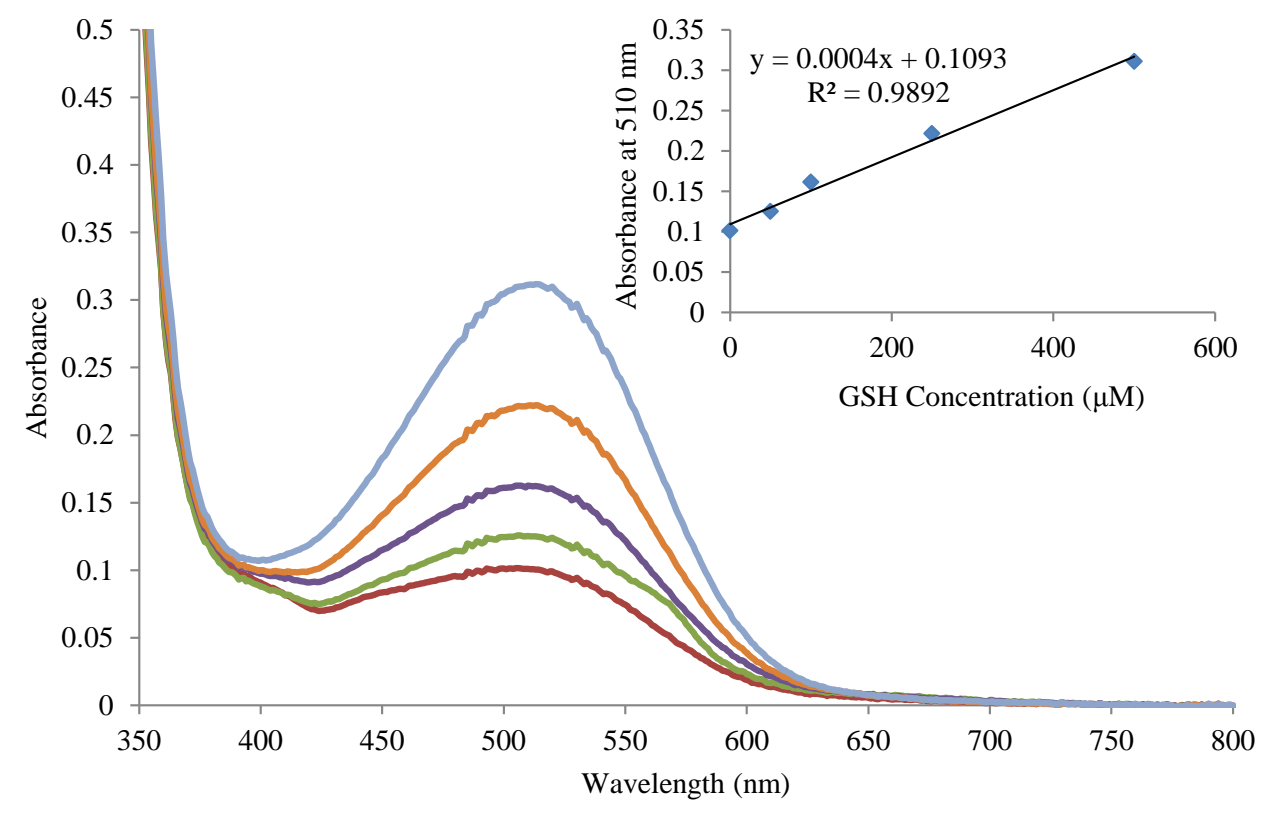

Figure 5.14. Absorption spectral changes of $\mathbf{2 4}$ upon addition of increasing concentrations of GSH (0-500 $\mu \mathrm{M})$. Inset: absorbance changes at $510 \mathrm{~nm}$ as a function of the concentrations of GSH. All solutions were composed of $200 \mathrm{mM}$ Tris buffer (pH 9) and $\mathbf{2 4}(500 \mu \mathrm{M})$.

To investigate the possible involvement of free radicals, EPR studies of the probe were carried out in the absence and presence of GSH. Interestingly, $\mathbf{2 4}$ alone produced an EPR signal of greater amplitude compared to the one generated in the presence of GSH (Figure 5.15). Thus, the presence of GSH diminished the amount of radical species that were generated by the probe itself. Although this experiment suggested the possibility of a free radical mechanism being involved, after samples were taken out from the EPR instrument, we observed precipitation, an indication that the data may not reflect the mechanism. Further investigations were performed including mass spectrometry. NMR studies were not possible because of solubility issues of the probe at higher concentrations needed. 




Figure 5.15 EPR spectrum of $\mathrm{PhCV}^{2+}(\mathbf{2 4}),(20 \mathrm{mM})$ in the presence and absence of $\mathrm{GSH}(10 \mathrm{mM})$ at $\mathrm{pH} 9$ (200 mM, Phosphate buffer).

Since Hcy selectivity would be more favorable if all thiols are forming the reducing, $\alpha$-amino carbon-centered radical, we propose that there are other factors enabling selectivity for GSH. These include favorable structural and/or electrostatic interactions between the probe (cationic) and anionic GSH. These also could promote supramolecular assembly formation. ${ }^{131}$

Molecular calculations (MM2, Gasteiger-Hückel charges) using the SYBYL-X ${ }^{\mathrm{TM}}$ (version 2.0, Certara, L.P.) suite reveal that the interactions of $\mathbf{2 4}$ with GSH are in fact, driven by electrostatics and the spatial configuration of $\mathbf{2 4}$ is dramatically modified as a result (Figure 5.16). 

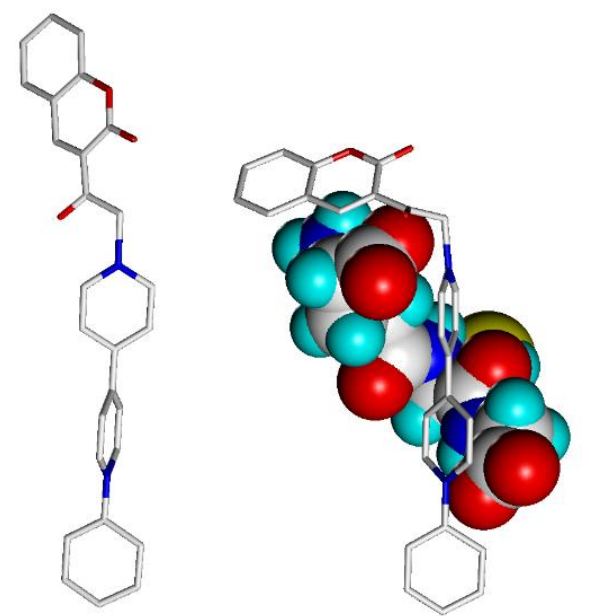

Figure 5.16. Energy minimized structures of $\mathbf{2 4}$ (left) and $\mathbf{2 4}$ in the presence of GSH (right).

\subsection{Experimental section}

\subsubsection{Materials and instruments}

All chemicals were purchased from Sigma-Aldrich or Acros and used without further purification. ${ }^{1} \mathrm{H}-\mathrm{NMR}$ and ${ }^{13} \mathrm{C}-\mathrm{NMR}$ spectra were recorded on a Bruker AMX-400 NMR spectrometer, using TMS as an internal standard. ESI-HRMS (high resolution mass spectrometry) spectra were obtained on a Thermo Electron LTQ Orbitrap hybrid mass spectrometer. UV-visible spectra were collected on a Cary 50 UV-Vis spectrophotometer; Fluorescence spectra were collected on a Cary Eclipse (Varian, Inc.) fluorescence spectrophotometer with slit widths set at $5 \mathrm{~nm}$ for both excitation and emission, respectively. The high voltage of the fluorescence spectrophotometer was set at $550 \mathrm{~V}$ and $\mathrm{pH}$ measurements were carried out with an Orion 410A pH meter. 


\subsubsection{Synthesis of the NIR acrylate 49}<smiles>Oc1cccc2cccc(O)c12</smiles>

54<smiles>O=C1OC(=O)c2ccccc21</smiles>

55



$71 \%$<smiles></smiles>

56

<smiles>C=CC(=O)Oc1cccc2ccc3c(c12)Oc1c(ccc2cccc(O)c12)C31C2C=CC(C2)C1(O)OC(=O)C=C</smiles>

49

Figure 5.17 Synthesis of NIR acrylate probe $\mathbf{5 1}$

\section{Improved synthesis of 1, 13-dihydroxy-3' H-spiro[dibenzo[c,h]xanthene-7,1'- isobenzofuran]-3'-one, 56}

This compound was previously synthesized by our group ${ }^{132}$ in $18 \%$ yield and here we modified the procedure by allowing the reaction to run for a longer time, $24 \mathrm{~h}$ compared to the previously used $16 \mathrm{~h}$. Additionally, commercially available $\mathbf{5 4}$ was used instead of synthesizing it. This resulted in a pure compound in high yield and didn't require purification by flash chromatography as before. 1,8-dihydroxy naphthalene, $\mathbf{5 4}$ $(0.5 \mathrm{~g}, 3.12 \mathrm{mmol})$, and phthalic anhydride $57(0.231 \mathrm{~g}, 1.56 \mathrm{mmol})$ are dissolved in 2 $\mathrm{mL}$ of methanesulfonic acid. To this solution is added $2 \mathrm{~mL}$ of TFA the mixture is heated 
and stirred at $80{ }^{\circ} \mathrm{C}$ for $24 \mathrm{~h}$. The reaction mixture is cooled to room temperature and then poured into $100 \mathrm{~mL}$ of DI water. The precipitated solid is filtered and washed with DI water $(5 \times 100 \mathrm{~mL})$ to give a pure grey compound which is air-dried overnight. Yield $0.958 \mathrm{~g}, 71 \% .{ }^{1} \mathrm{H}$ NMR (400 MHz, DMSO) $\delta 11.34$ (s, 2H), $8.14-8.09(\mathrm{~m}, 1 \mathrm{H}), 7.82-$ $7.75(\mathrm{~m}, 2 \mathrm{H}), 7.62(\mathrm{~d}, J=8.8 \mathrm{~Hz}, 2 \mathrm{H}), 7.53(\mathrm{t}, J=7.8 \mathrm{~Hz}, 2 \mathrm{H}), 7.44(\mathrm{~d}, J=7.5 \mathrm{~Hz}, 2 \mathrm{H})$, $7.39(\mathrm{~d}, J=6.8 \mathrm{~Hz}, 1 \mathrm{H}), 7.12(\mathrm{~d}, J=6.9 \mathrm{~Hz}, 2 \mathrm{H}), 6.75(\mathrm{~d}, J=8.7 \mathrm{~Hz}, 2 \mathrm{H})$.

\section{1-hydroxy-3'-oxo-3'H-spiro[dibenzo[c,h]xanthene-7,1'-isobenzofuran]-13-yl acrylate, 51}

Under argon compound $51(0.200 \mathrm{~g}, 0.463 \mathrm{mmol})$ and $\mathrm{Et}_{3} \mathrm{~N}(0.129 \mathrm{~mL}, 0.925$ mmol) are dissolved in $15 \mathrm{~mL}$ anhydrous DCM, then acryloyl chloride (0.094 mL, 1.16 mmol) in $5 \mathrm{~mL}$ DCM is added dropwise at $0^{\circ} \mathrm{C}$. The reaction mixture is stirred at this temperature for $1 \mathrm{~h}$, then warmed to $\mathrm{rt}$ and stirred overnight. The resulting mixture is diluted with DCM $(20 \mathrm{~mL})$, washed with water $(20 \mathrm{~mL} \times 3)$ and dried over anhydrous $\mathrm{Na}_{2} \mathrm{SO}_{4}$. The solvent is removed in vacuo and the target compound is isolated by flash column chromatography on silica gel using $\mathrm{CH}_{2} \mathrm{Cl}_{2}$ : MeOH 98:2 for elution to yield a cream powder $0.128 \mathrm{~g}, 57 \% .{ }^{1} \mathrm{H}$ NMR $(400 \mathrm{MHz}, \mathrm{CDCl} 3) \delta 9.41(\mathrm{~s}, 1 \mathrm{H}), 8.12-8.07$ (m, 1H), $7.80(\mathrm{~d}, J=7.5 \mathrm{~Hz}, 1 \mathrm{H}), 7.68-7.59(\mathrm{~m}, 4 \mathrm{H}), 7.53-7.47(\mathrm{~m}, 2 \mathrm{H}), 7.40-7.33(\mathrm{~m}$, 2H), $7.18-7.12(\mathrm{~m}, 2 \mathrm{H}), 7.00-6.91(\mathrm{~m}, 2 \mathrm{H}), 6.79(\mathrm{~d}, J=8.7 \mathrm{~Hz}, 1 \mathrm{H}), 6.60(\mathrm{dd}, J=$ 17.3, $1.3 \mathrm{~Hz}, 1 \mathrm{H}), 6.01(\mathrm{dd}, J=10.4,1.3 \mathrm{~Hz}, 1 \mathrm{H}) .{ }^{13} \mathrm{C} \mathrm{NMR}(101 \mathrm{MHz}, \mathrm{CDCl} 3) \delta$ $169.40,165.43,153.80,153.28,147.12,145.58,145.19,136.57,135.44,134.25,130.12$, $129.15,127.15,127.06,125.80,125.56,124.23,124.08,123.26,122.03,119.59,117.84$ 
$114.51,113.39,112.93,112.50,82.08$. HR ESI $[\mathrm{M}+\mathrm{H}]^{+}, m / z: 487.1182$ calc for $\mathrm{C}_{31} \mathrm{H}_{19} \mathrm{O}_{6}{ }^{+} 487.1186$.

5.4.3 CTAB-mediated spectral responses of acrylate probes towards thiols general procedure.

The acrylate probe is added to solutions of thiols in $2.0 \mathrm{mM}$ CTAB media buffered at pH 7.4 (phosphate buffer, $50 \mathrm{mM}$ ). Spectra (UV-Vis and Fluorescence) were collected immediately upon addition of the probe. In the case of deproteinized plasma, its proportion is $10 \%$ of the total volume after being mixed with the CTAB, buffer and probe solutions.

\subsection{Conclusion}

Probe 52 can function as a simple new indicator for the highly selective and sensitive detection of GSH. This embodies a sensing mechanism that couples a conjugate addition reaction and a micelle-promoted 12-membered ring formation/elimination sequence. Due to the lack of fluorescence probes for the highly selective detection of GSH over other thiols in blood plasma, the discovery of $\mathbf{5 2}$ is highly significant. A new class of fluorescent probes for GSH may be developed through this new mechanism.

We have also developed a viologen probe $\mathbf{2 4}$ for selective detection of GSH without any interference from structurally related analytes via a binding interaction. This probe has potential for quantifying GSH as demonstrated by the linear relationship between 24 and GSH concentration. 
CHAPTER.6: TOWARDS DEVELOPING A METHOD FOR DETECTION AND QUANTITATION OF GSH IN WHOLE BLOOD DEPOSITED ON FILTER PAPER

\subsection{Introduction}

There is often not a clear correlation between whole blood GSH and plasma GSH levels. This has been seen for example in the initial phase of sepsis where plasma concentrations of GSH decreased, whereas GSH status in skeletal muscle and whole blood remain unaltered. ${ }^{133}$ In contrast, in another recent study investigating temporal changes in whole blood and plasma glutathione in hospital ICU patients with multiple organ failure, GSH levels from whole blood and not plasma were depleted in critically ill patients with chronic obstructive pulmonary disease. ${ }^{134}$ Levels of whole-blood glutathione in peritoneal dialysis patients have been used to monitor therapeutic outcomes. ${ }^{135}$ It is more efficient to use a whole blood GSH to diagnose mitochondrial disorders, as whole blood GSH levels are ca. 1000-fold higher than blood plasma levels.

Moreover, it has been noted that whole blood GSH measurements generally minimize variability caused by sample preparation steps. ${ }^{136}$ In general, whole blood GSH levels are indicative of the pathogenesis of major diseases that are often distinct from those that can be monitored using plasma GSH. Therefore, there is a need to develop simple methods that can detect and quantify GSH in whole blood.

The resorufin-acrylate probe though successful it has its own drawback that it's a short wavelength probe, hence its efficient function in a whole blood matrix would be hampered by optical interferences from hemoglobin $(\mathrm{Hb})$. Several years ago, extreme 
sample dilution (e.g. 5000-fold) was used to minimize hemoglobin interference in the determination of GSH in dried blood spots on filter paper. ${ }^{137} \mathrm{Hb}$ content would be reduced to less than $30 \mu \mathrm{g} / \mathrm{mL}$ reducing absorbance near $415 \mathrm{~nm}$ to about 0.25 in a standard $1 \mathrm{~cm}$ cell. Dried blood specimens on filter paper are easy to obtain and work with and present much fewer logistical problems as compared to using plasma or whole blood samples. Only a small volume of blood is required. Disks are punched from the paper and the blood contents typically eluted/extracted into a small volume of solvent.

Apart from dilution, $\mathrm{Hb}$ can be removed using a commercial product, HemogloBind. This product can isolate and remove up to $90 \%$ of blood $\mathrm{Hb} .{ }^{138}$ However, this still requires some dilution (500-fold dilution) and even with $10 \% \mathrm{Hb}$ remaining after HemogloBind processing, there is still $\mathrm{Hb}$ spectral overlap.

The work herein entails a new and relatively simple method for detection and quantitation of GSH in whole blood deposited on filter paper without interference from Hb. ${ }^{124}$ The method involves deproteinization of blood extracted from a filter paper with concomitant fractionation of GSH using size exclusion chromatography (Figure 6.1). In the process $\mathrm{Hb}$ would be completely removed from the fraction containing GSH. This would be an ideal method for drawing small sample volumes, which are easy to obtain from children and for enabling shipment of samples to a centralized lab. A small volume of blood dries quickly on paper as cells rupture, emptying their GSH stores. The method is also suitable in developing nations with limited access to refrigeration or electricity and in developed nations due to the rising healthcare costs and the high interest in hometesting kits. 


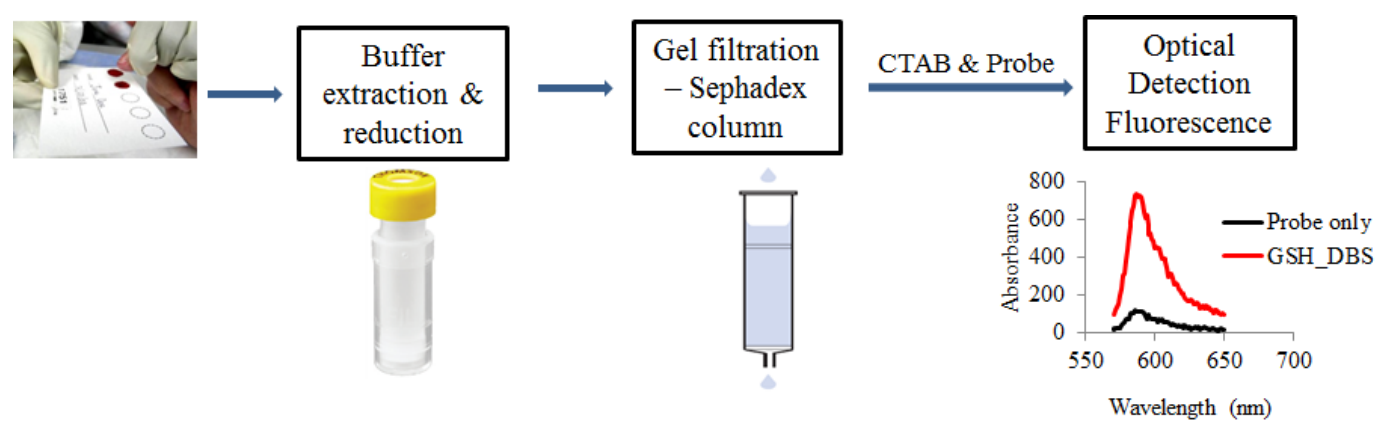

Figure 6.1. A method for detection of GSH in whole blood deposited on a filter paper ${ }^{124}$

Another advantage of this method is that deproteinization is carried out without using acids and organic solvents that can adversely affect biomolecules. Compared to the use of acids and organic solvents, filtration is a more benign method of removing proteins. The use of filters may also minimize the oxidation of GSH to GSSG. ${ }^{139,140}$

\subsection{Results and discussion}

We successfully managed to show proof of concept that GSH can be fractionated away from proteins, $\mathrm{Hb}$ and other potentially interfering blood components very efficiently from a blood spot. Upon passing the extracted GSH from the dried blood spot (DBS) through the PD MiniTrap ${ }^{\mathrm{TM}}$ G-25 Sephadex column, all proteins elute in fractions 1, 2 and 3. This was confirmed by running UV-Vis absorption of the fractions and monitoring the peak at $290 \mathrm{~nm}$ (Figure 6.2). Only fractions 1, 2 and 3 exhibited significant absorption responses and none was observed in fractions 4 to 6 . The other peaks at $400 \mathrm{~nm}$, and 500-600 $\mathrm{nm}$ are a result of $\mathrm{Hb}$ which is highly absorbing. It can be 
observed that these peaks are absent in fractions 4, 5 and 6 suggesting that if GSH elutes in these fractions there wouldn't be any interference from $\mathrm{Hb}$.

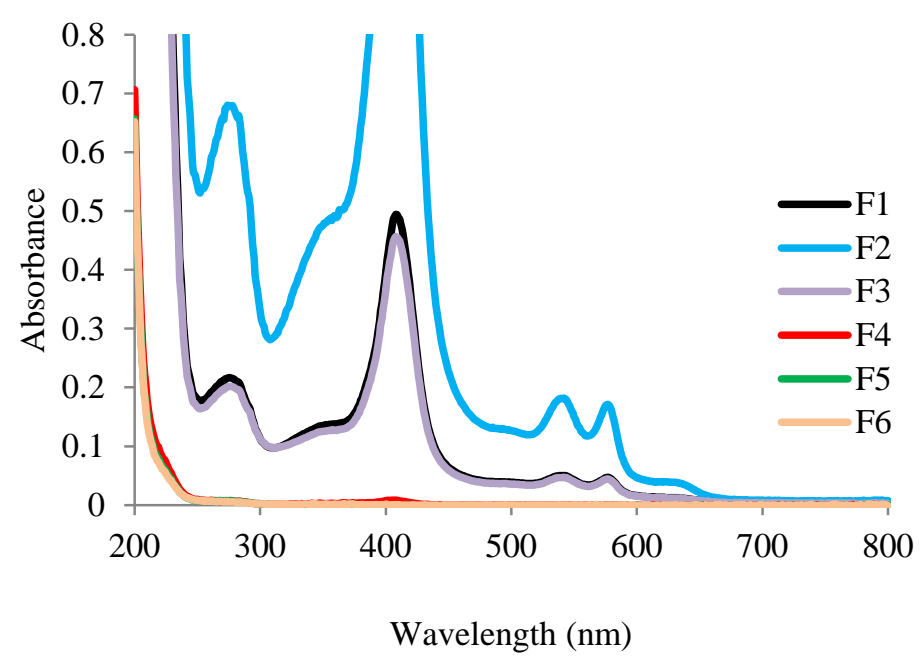

Figure 6.2. Absorption responses of DBS fractions in phosphate buffer at $\mathrm{pH} 7.4$ Fractions were collected and immediately analyzed for absorption.

Fractions 4, 5 and 6 were then tested for the presence of GSH using the resorufinacrylate CTAB system. Upon addition of probe $\mathbf{5 0}(2.5 \mu \mathrm{M})$ to the fractions in $2.0 \mathrm{mM}$ CTAB media buffered at $\mathrm{pH} 7.4$ (phosphate buffer), significant fluorescence responses were observed in fractions 4 and 5 indicating the presence of native GSH in blood (Figure 6.3). The high responses in fractions 1,2 and 3 are a result of the reaction of the probe with proteins. Fraction 6 behaved in the same manner as the blank. 




Figure 6.3 Spectral response of $\mathbf{5 0}$ towards various DBS fractions at $\mathrm{pH}$ 7.4. Fluorescence spectra $(\lambda \mathrm{ex}=$ $565 \mathrm{~nm})$ of $\mathbf{5 0}(2.5 \mu \mathrm{M})$ in solutions of DBS fractions and $2.0 \mathrm{mM}$ CTAB media buffered at $\mathrm{pH} 7.4$ (phosphate buffer, $50 \mathrm{mM}$ ). Spectra were taken immediately upon addition of the probe.

To further confirm that fractions 4 and 5 indeed contained GSH, we examined commercial, standard GSH and collected all the six fractions. Upon addition of the probe and $\mathrm{CTAB}$ only fractions 4 and 5 developed a pink color as well as generating a significant fluorescence response as shown in figure A9 (Appendix A). Other fractions 1, 2 and 3 where proteins elute remained unchanged and their fluorescence responses were the same as the blank.

To investigate the effectiveness of this gel filtration method in deproteinizing dried blood, control experiments were performed using human serum albumin (HSA) at its whole blood concentrations $(60 \mathrm{mg} / \mathrm{mL})$. As expected no responses were observed in fractions 1, 2 and 3, confirming the absence of proteins in these fractions (Figure 6.4). This effectively means that the fluorescence responses observed in fractions 4 and 5 were due to native GSH and not residual proteins. 


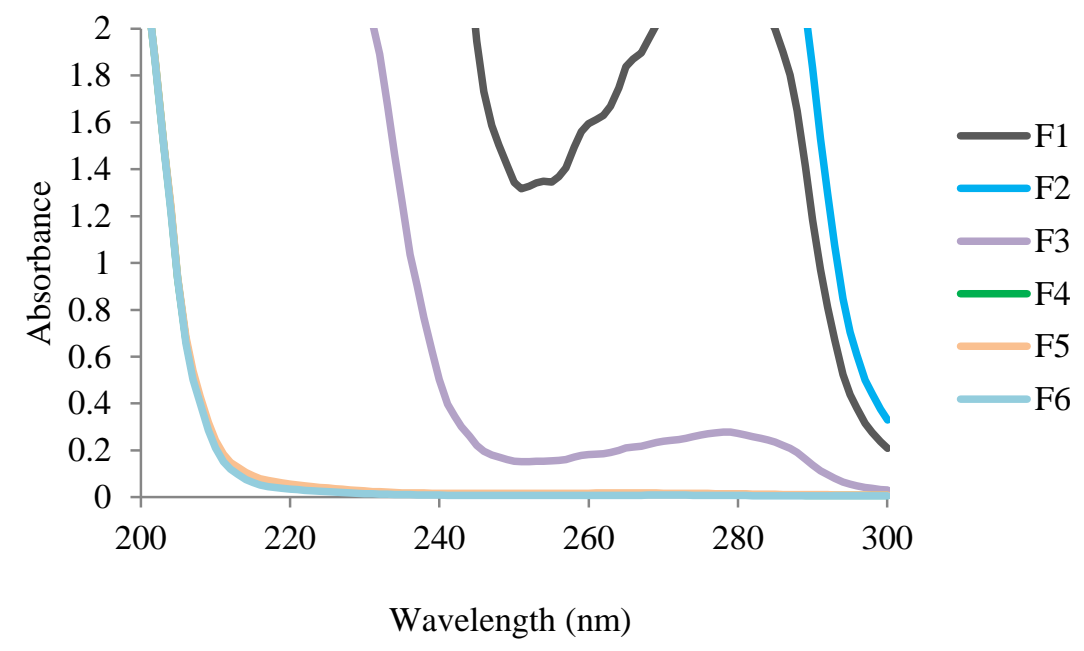

Figure.6.4 Absorption responses of HSA fractions in phosphate buffer at pH 7.4 Fractions were collected and immediately analyzed for absorption.

Another control experiment to rule out any interference from $\mathrm{Hb}$ was conducted using commercial $\mathrm{Hb}(20 \mathrm{~g} / \mathrm{dL}) . \mathrm{Hb}$ was eluted together with proteins in fractions 1,2 and 3 (Figure 6.5). No absorption response to $\mathrm{Hb}$ was seen in fractions 4, 5 and 6.



Figure 6.5 Absorption responses of $\mathrm{Hb}$ fractions in phosphate buffer at $\mathrm{pH} 7.4$ Fractions were collected and immediately analyzed for absorption. 
The same fractions were analyzed for their fluorescence response and as expected, upon addition of the probe and CTAB, no response was observed in fractions 4,5 and 6 showing that this method completely eliminated all $\mathrm{Hb}$ (Figure 6.6). Any shorter wavelength probe that can selectively detect GSH in the absence of proteins and $\mathrm{Hb}$ can be applied with this method. We selected the resorufin-acrylate probe (50) over $\mathrm{PhCV}^{2+}$ (24) due to its very low detection limit and its fast response.



Figure 6.6 Spectral response of $\mathbf{5 0}$ towards various $\mathrm{Hb}$ fractions at $\mathrm{pH}$ 7.4. Fluorescence spectra $(\lambda \mathrm{ex}=565$ $\mathrm{nm})$ of $\mathbf{5 0}(2.5 \mu \mathrm{M})$ in solutions of DBS fractions and $2.0 \mathrm{mM}$ CTAB media buffered at $\mathrm{pH} 7.4$ (phosphate buffer, $50 \mathrm{mM}$ ). Spectra were taken immediately upon addition of the probe.

We optimized our method to increase the GSH signal from the DBS by increasing the drying time from 3 hours to 24 hours (overnight). Interestingly, as shown in figure 6.7, the GSH signal increased significantly upon addition of the probe and CTAB. We also noticed the difference in the color of the filter papers spotted at these different times with the overnight filter paper looking completely dry. This was attributed to the 
complete rupture of the erythrocytes empting more GSH stores. For further studies, a drying time of 24 hours was used.

(a)

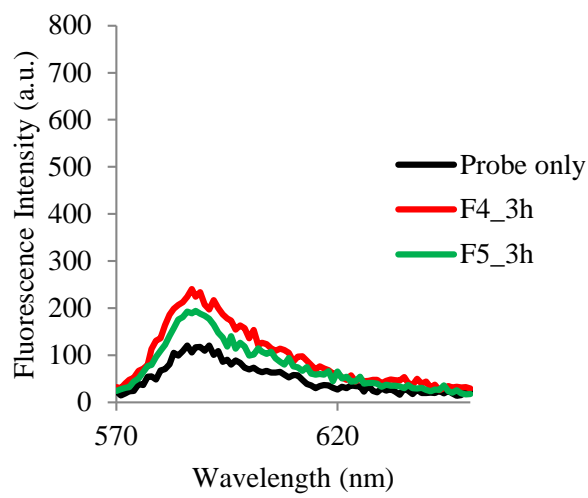

(b)

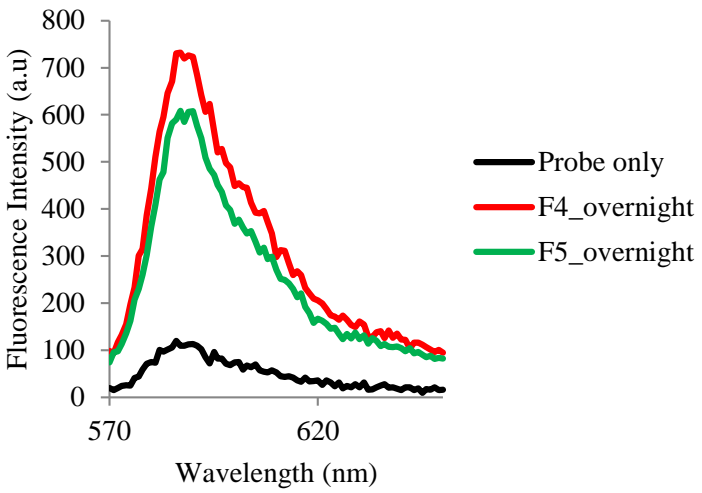

Figure 6.7 Spectral response of $\mathbf{5 0}$ towards various DBS fractions at $\mathrm{pH}$ 7.4. Fluorescence spectra $(\lambda \mathrm{ex}=$ $565 \mathrm{~nm})$ of $\mathbf{5 0}(2.5 \mu \mathrm{M})$ in solutions of DBS fractions from blood dried at different times and $2.0 \mathrm{mM}$ CTAB media buffered at $\mathrm{pH} 7.4$ (phosphate buffer, $50 \mathrm{mM}$ ). Spectra were taken immediately upon addition of the probe. (a) Blood dried for $3 \mathrm{~h}$ (b) Blood dried for $24 \mathrm{~h}$.

Having identified the optimal conditions for GSH detection without any interference from proteins, $\mathrm{Hb}$ and other blood components, we investigated the potential of this method in quantifying GSH. The fluorescence response of 50-CTAB system towards fractionated GSH increased linearly with incremental addition of GSH over a physiological relevant range $(0-2 \mathrm{mM})$ (Figure 6.8 (a) and (b)). The concentration of GSH in a DBS of pig blood was determined to be $1.825 \mathrm{mM}$ by standard addition which falls within the range of reported intracellular GSH levels (1-2 mM). 



Figure 6.8 Spectral response of $\mathbf{5 0}$ towards DBS spiked with various concentrations of GSH. (a). Fluorescence spectra $(\lambda \mathrm{ex}=565 \mathrm{~nm})$ of $\mathbf{5 0}$ with increasing spiked GSH concentration $(0-2 \mathrm{mM})$. (b) Calibration curve for $\mathbf{5 0}$ showing linearity between fluorescence intensity and spiked DBS with GSH. All solutions contained $50(2.5 \mu \mathrm{M}), 2.0 \mathrm{mM}$ CTAB media and $50 \mathrm{mM}$ phosphate buffer $(\mathrm{pH} 7.4)$.

During to the spotting and filtration process some GSH is lost. We evaluated this by comparing the fluorescence responses of standard GSH that underwent the entire process of spotting, extraction, reduction and filtration against one which didn't undergo the process. As shown in figure 6.9, more than $70 \%$ of native GSH from the DBS is recovered in this method, however the process can be optimized to reduce the losses. 


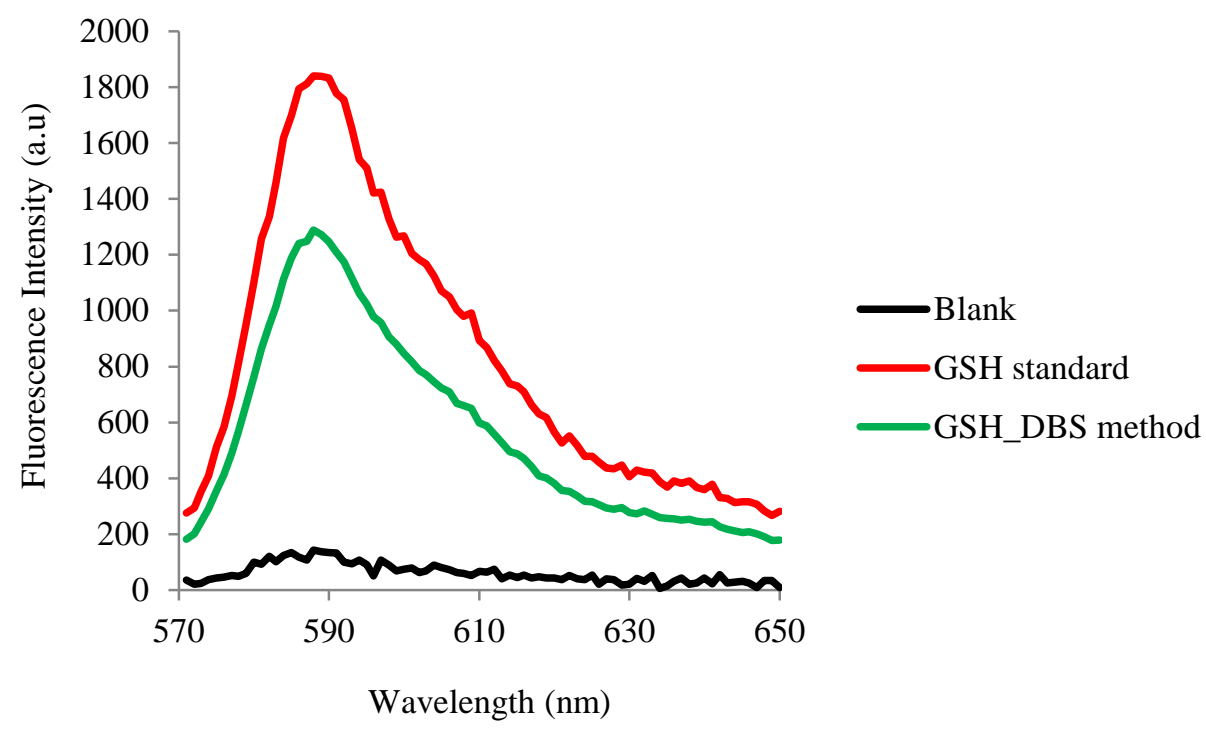

Figure 6.9 Comparison of commercial GSH fractions vs non-fractionated GSH at $\mathrm{pH}$ 7.4. Fluorescence spectra $(\lambda e x=565 \mathrm{~nm})$ of $\mathbf{5 0}(2.5 \mu \mathrm{M})$ in solutions of GSH fractions from commercial GSH (spotted, dried, extracted, reduced and separated by the column) and $2.0 \mathrm{mM} \mathrm{CTAB}$ media buffered at $\mathrm{pH} 7.4$ (phosphate buffer, $50 \mathrm{mM}$ ). Spectra were taken immediately upon addition of the probe.

\subsection{Experimental section}

\subsubsection{Fractionation of GSH}

In this method blood is spotted on the filter paper and dried overnight, followed by extraction with buffer. Upon drying blood, cells are ruptured releasing GSH which in turn becomes oxidized to GSSG. Once oxidized, it is stable in dried blood specimens and can be assayed at a later time. In this assay the oxidized glutathione (GSSG) is reduced to GSH by treating it with the immobilized tris(2-carboxyethyl)phosphine (TCEP gel), a disulfide reducing agent. The reduced sample is passed through the PD MiniTrap ${ }^{\text {TM }}$ G-25 Sephadex column where GSH is fractionated away from proteins, $\mathrm{Hb}$ and other potentially interfering blood components. 
6.3.2 CTAB-mediated spectral responses of acrylate probes to GSH fractions.

The acrylate probe is added to fractionated solutions in $2.0 \mathrm{mM} \mathrm{CTAB}$ media buffered at pH 7.4 (phosphate buffer, $50 \mathrm{mM}$ ). Spectra (UV-Vis and Fluorescence) were collected immediately upon addition of the probe.

\subsection{Conclusion}

We have developed a new separation technique wherein glutathione can be fractionated away from proteins and other potentially interfering blood components very efficiently. This method eliminates the need to use very expensive materials like hemoglobind to remove $\mathrm{Hb}$ and allows for the use of shorter wavelengths probes to determine GSH in natural media. This assay has potential for quantitation of GSH in whole blood as demonstrated by the linear calibration curve. 


\section{REFERENCES}

1. Forman, H. J.; Zhang, H. Q.; Rinna, A. "Glutathione: Overview of its protective roles, measurement, and biosynthesis," Mol. Asp. Med. 2009, 30, 1-12.

2. Valko, M.; Leibfritz, D.; Moncol, J.; Cronin, M. T. D.; Mazur, M.; Telser, J. "Free radicals and antioxidants in normal physiological functions and human disease," Int. J. Biochem. Cell Biol. 2007, 39, 44-84.

3. Circu, M. L.; Yee Aw, T. "Glutathione and apoptosis," Free Radic. Res. 2008, 42, 689-706.

4. Meister, A. "Glutathione metabolism and its selective modification," J. Biol. Chem. 1988, 263, 17205-17208.

5. Camera, E.; Picardo, M. "Analytical methods to investigate glutathione and related compounds in biological and pathological processes," J. Chromatogr. B 2002, 781, 181-206.

6. Ferin, R.; Pavao, M. L.; Baptista, J. "Methodology for a rapid and simultaneous determination of total cysteine, homocysteine, cysteinylglycine and glutathione in plasma by isocratic RP-HPLC," J. Chromatogr. B 2012, 911, 15-20.

7. Ueland, P. M. "Homocysteine species as components of plasma redox thiol status," Clin. Chem. 1995, 41, 340-342.

8. Mansoor, M. A.; Svardal, A. M.; Ueland, P. M. "Determination of the in vivo redox status of cysteine, cysteinylglycine, homocysteine, and glutathione in human plasma," Anal. Biochem. 1992, 200, 218-229. 
9. Jacobsen, D. W.; Gatautis, V. J.; Green, R.; Robinson, K.; Savon, S. R.; Secic, M.; Ji, J.; Otto, J. M.; Taylor, L. M. "Rapid HPLC determination of total homocysteine and other thiols in serum and plasma - sex-differences and correlation with cobalamin and folate concentrations in healthy subjects," Clin. Chem. 1994, 40, 873-881.

10. Isokawa, M.; Kanamori, T.; Funatsu, T.; Tsunoda, M. "Analytical methods involving separation techniques for determination of low-molecular-weight biothiols in human plasma and blood," J. Chromatogr. B 2014, 964, 103-115.

11. Shahrokhian, S. "Lead phthalocyanine as a selective carrier for preparation of a cysteine-selective electrode," Anal. Chem. 2001, 73, 5972-5978.

12. Selhub, J. "Homocysteine metabolism," Annu. Rev. Nutr. 1999, 19, 217-246.

13. He, M.; Johnson, R. J.; Escobedo, J. O.; Beck, P. A.; Kim, K. K.; St. Luce, N. N.; Davis, C. J.; Lewis, P. T.; Fronczek, F. R.; Melancon, B. J.; Mrse, A. A.; Treleaven, W. D.; Strongin, R. M. "Chromophore formation in resorcinarene solutions and the visual detection of mono- and oligosaccharides," J. Am. Chem. Soc. 2002, 124, 5000-5009.

14. Glushchenko, A. V.; Jacobsen, D. W. "Molecular targeting of proteins by Lhomocysteine: Mechanistic implications for vascular disease," Antioxid. Redox Signal. 2007, 9, 1883-1898.

15. Werder, S. F. "Cobalamin deficiency, hyperhomocysteinemia, and dementia," Neuropsychiatr. Dis. Treat. 2010, 6, 159-195. 
16. Malinow, M. R.; Bostom, A. G.; Krauss, R. M. "Homocyst(e)ine, diet, and cardiovascular diseases - A statement for healthcare professionals from the Nutrition Committee, American Heart Association," Circulation 1999, 99, 178182.

17. Rusin, O.; Alpturk, O.; He, M.; Escobedo, J. O.; Jiang, S.; Dawan, F.; Lian, K.; McCarroll, M. E.; Warner, I. M.; Strongin, R. M. "Macrocycle-derived functional xanthenes and progress towards concurrent detection of glucose and fructose," $J$. Fluoresc. 2004, 14, 611-615.

18. Robinson, K. "Homocysteine, B vitamins, and risk of cardiovascular disease," Heart 2000, 83, 127-130.

19. Morgenstern, I.; Raijmakers, M. T. M.; Peters, W. H. M.; Hoensch, H.; Kirch, W. "Homocysteine, cysteine, and glutathione in human colonic mucosa - Elevated levels of homocysteine in patients with inflammatory bowel disease," Dig. Dis. Sci. 2003, 48, 2083-2090.

20. Refsum, H.; Ueland, P. M.; Nygard, O.; Vollset, S. E. Homocysteine and cardiovascular disease. In Annu. Rev. Med.; Coggins, C. H., Ed. 1998; Vol. 49, p $31-62$.

21. Seshadri, S.; Beiser, A.; Selhub, J.; Jacques, P. F.; Rosenberg, I. H.; D'Agostino, R. B.; Wilson, P. W. F.; Wolf, P. A. "Plasma homocysteine as a risk factor for dementia and Alzheimer's disease," N. Engl. J. Med. 2002, 346, 476-483.

22. Strain, J. J.; Dowey, L.; Ward, M.; Pentieva, K.; McNulty, H. "B-vitamins, homocysteine metabolism and CVD," Proc. Nutr. Soc. 2004, 63, 597-603. 
23. Bleich, S.; Carl, M.; Bayerlein, K.; Reulbach, U.; Biermann, T.; Hillemacher, T.; Bonsch, D.; Kornhuber, J. "Evidence of increased homocysteine levels in alcoholism: The Franconian Alcoholism Research Studies (FARS)," AlcoholismClinical and Experimental Research 2005, 29, 334-336.

24. Gao, X. H.; Zhang, G. Y.; Wang, Y.; Zhang, H. Y. "Correlations of MTHFR 677C > T polymorphism with cardiovascular disease in patients with end-stage renal disease: a meta-analysis," PLOS ONE 2014, 9.

25. Peyrin-Biroulet, L.; Rodriguez-Gueant, R. M.; Chamaillard, M.; Desreumaux, P.; Xia, B.; Bronowicki, J. P.; Bigard, M. A.; Gueant, J. L. "Vascular and cellular stress in inflammatory bowel disease: Revisiting the role of homocysteine," Am. J. Gastroenterol. 2007, 102, 1108-1115.

26. De Bree, A.; Verschuren, W. M. M.; Kromhout, D.; Kluijtmans, L. A. J.; Blom, H. J. "Homocysteine determinants and the evidence to what extent homocysteine determines the risk of coronary heart disease," Pharmacol. Rev. 2002, 54, 599618.

27. Brachet, P.; Chanson, A.; Demigne, C.; Batifoulier, F.; Alexandre-Gouabau, M. C.; Tyssandier, V.; Rock, E. "Age-associated B vitamin deficiency as a determinant of chronic diseases," Nutr. Res. Rev. 2004, 17, 55-68.

28. Alfthan, G.; Aro, A.; Gey, K. F. "Plasma homocysteine and cardiovascular disease mortality," Lancet 1997, 349, 397-397.

29. Vollset, S. E.; Refsum, H.; Tverdal, A.; Nygard, O.; Nordrehaug, J. E.; Tell, G. S.; Ueland, P. M. "Plasma total homocysteine and cardiovascular and 
noncardiovascular mortalily: the Hordaland Homocysteine Study," Am. J. Clin. Nutr. 2001, 74, 130-136.

30. Wald, D. S.; Law, M.; Morris, J. K. "Homocysteine and cardiovascular disease: evidence on causality from a meta-analysis," Br. Med. J. 2002, 325, 1202-1206K.

31. Homocysteine Studies, C. "Homocysteine and risk of ischemic heart disease and stroke - A meta-analysis," JAMA-J. Am. Med. Assoc. 2002, 288, 2015-2022.

32. Bertoia, M. L.; Pai, J. K.; Cooke, J. P.; Joosten, M. M.; Mittleman, M. A.; Rimm, E. B.; Mukamal, K. J. "Plasma homocysteine, dietary B vitamins, betaine, and choline and risk of peripheral artery disease," Atherosclerosis 2014, 235, 94-101.

33. Veeranna, V.; Zalawadiya, S. K.; Niraj, A.; Pradhan, J.; Ference, B.; Burack, R. C.; Jacob, S.; Afonso, L. "Homocysteine and reclassification of cardiovascular disease risk," J. Am. Coll. Cardiol. 2011, 58, 1025-1033.

34. Xu, H. P.; Liu, C. M.; Wang, Q. H. "Plaque image characteristics, hyperhomocysteinemia, and gene polymorphism of homocysteine metabolismrelated enzyme (MTHFR C677T) in acute coronary syndrome," Cell Biochem. Biophys. 2013, 66, 403-407.

35. Klerk, M.; Verhoef, P.; Clarke, R.; Blom, H. J.; Kok, F. J.; Schouten, E. G.; Grp, M. S. C. "MTHFR 677C -> T polymorphism and risk of coronary heart disease A meta-analysis," JAMA-J. Am. Med. Assoc. 2002, 288, 2023-2031.

36. Lewis, S. J.; Ebrahim, S.; Smith, G. D. "Meta-analysis of MTHFR 677C -> T polymorphism and coronary heart disease: does totality of evidence support 
causal role for homocysteine and preventive potential of folate?," $\mathrm{Br}$. Med. J. 2005, 331, 1053-1056A.

37. Brustolin, S.; Giugliani, R.; Felix, T. M. "Genetics of homocysteine metabolism and associated disorders," Braz. J. Med. Biol. Res. 2010, 43, 1-7.

38. Refsum, H.; Smith, A. D.; Ueland, P. M.; Nexo, E.; Clarke, R.; McPartlin, J.; Johnston, C.; Engbaek, F.; Schneede, J.; McPartlin, C.; Scott, J. M. "Facts and recommendations about total homocysteine determinations: An expert opinion," Clin. Chem. 2004, 50, 3-32.

39. Selhub, J. "Public health significance of elevated homocysteine," Food Nutr. Bull. 2008, 29, S116-S125.

40. Lentz, S. R.; Haynes, W. G. "Homocysteine: is it a clinically important cardiovascular risk factor?," Clevel. Clin. J. Med. 2004, 71, 729-734.

41. Kaluzna-Czaplinska, J.; Zurawicz, E.; Michalska, M.; Rynkowski, J. "A focus on homocysteine in autism," Acta Biochim. Pol. 2013, 60, 137-142.

42. Yang, Q. H.; Botto, L. D.; Erickson, J. D.; Berry, R. J.; Sambell, C.; Johansen, H.; Friedman, J. M. "Improvement in stroke mortality in Canada and the United States, 1990 to 2002," Circulation 2006, 113, 1335-1343.

43. de Ruijter, W.; Westendorp, R. G. J.; Assendelft, W. J. J.; den Elzen, W. P. J.; de Craen, A. J. M.; le Cessie, S.; Gussekloo, J. "Use of Framinghamrisk score and new biomarkers to predict cardiovascular mortality in older people: population based observational cohort study," Br. Med. J. 2009, 338, a3083. 
44. Huo, Y.; Qin, X.; Wang, J.; Sun, N.; Zeng, Q.; Xu, X.; Liu, L.; Xu, X.; Wang, X. "Efficacy of folic acid supplementation in stroke prevention: new insight from a meta-analysis," Int. J. Clin. Pract. 2012, 66, 544-551.

45. Marti-Carvajal, A. J.; Sola, I.; Lathyris, D.; Salanti, G. "Homocysteine lowering interventions for preventing cardiovascular events," Cochrane Database Syst. Rev. 2009.

46. Jardine, M. J.; Kang, A.; Zoungas, S.; Navaneethan, S. D.; Ninomiya, T.; Nigwekar, S. U.; Gallagher, M. P.; Cass, A.; Strippoli, G.; Perkovic, V. "The effect of folic acid based homocysteine lowering on cardiovascular events in people with kidney disease: systematic review and meta-analysis," Br. Med. J. 2012, 344, e3533.

47. Smulders, Y. M.; Blom, H. J. "The homocysteine controversy," J. Inherit. Metab. Dis. 2011, 34, 93-99.

48. Smith, D. E. C.; Hornstra, J. M.; Kok, R. M.; Blom, H. J.; Smulders, Y. M. "Folic acid supplementation does not reduce intracellular homocysteine, and may disturb intracellular one-carbon metabolism," Clin. Chem. Lab. Med. 2013, 51, 16431650.

49. Cacciapuoti, F. "Lowering homocysteine levels with folic acid and B-vitamins do not reduce early atherosclerosis, but could interfere with cognitive decline and Alzheimer's disease," J. Thromb. Thrombolysis 2013, 36, 258-262.

50. Zappacosta, B.; Mastroiacovo, P.; Persichilli, S.; Pounis, G.; Ruggeri, S.; Minucci, A.; Carnovale, E.; Andria, G.; Ricci, R.; Scala, I.; Genovese, O.; Turrini, 
A.; Mistura, L.; Giardina, B.; Iacoviello, L. "Homocysteine lowering by folaterich diet or pharmacological supplementations in subjects with moderate hyperhomocysteinemia," Nutrients 2013, 5, 1531-1543.

51. McNulty, H.; Pentieva, K.; Hoey, L.; Ward, M. "Homocysteine, B-vitamins and CVD," Proc. Nutr. Soc. 2008, 67, 232-237.

52. Vasan, R. S. "Biomarkers of cardiovascular disease - Molecular basis and practical considerations," Circulation 2006, 113, 2335-2362.

53. Debreceni, B.; Debreceni, L. "The role of homocysteine-lowering B-vitamins in the primary prevention of cardiovascular disease," Cardiovasc. Ther. 2014, 32, $130-138$.

54. Wang, X. B.; Qin, X. H.; Demirtas, H.; Li, J. P.; Mao, G. Y.; Huo, Y.; Sun, N. L.; Liu, L. H.; Xu, X. P. "Efficacy of folic acid supplementation in stroke prevention: a meta-analysis," Lancet 2007, 369, 1876-1882.

55. La'ulu, S. L.; Rawlins, M. L.; Pfeiffer, C. M.; Zhang, M. D.; Roberts, W. L. "Performance characteristics of six homocysteine assays," Am. J. Clin. Pathol. 2008, 130, 969-975.

56. Valente, A.; Bronze, M. R.; Bicho, M.; Duarte, R.; Costa, H. S. "Validation and clinical application of an UHPLC method for simultaneous analysis of total homocysteine and cysteine in human plasma," J. Sep. Sci. 2012, 35, 3427-3433.

57. Nekrassova, O.; Lawrence, N. S.; Compton, R. G. "Analytical determination of homocysteine: a review," Talanta 2003, 60, 1085-1095. 
58. Newton, L. A. A.; Sandhu, K.; Livingstone, C.; Leslie, R.; Davis, J. "Clinical diagnostics for homocysteine: a rogue amino acid?," Expert Rev. Mol. Diagn. 2010, 10, 489-500.

59. Zappacosta, B.; Persichilli, S.; Minucci, A.; Scribano, D.; Baroni, S.; Fasanella, S.; Neri, P.; Daloiso, P. D.; Giardina, B.; De Sole, P. "Evaluation of a new enzymatic method for homocysteine measurement," Clin. Biochem. 2006, 39, 6266.

60. Ji, J. Z.; Meng, Q. H. "Evaluation of the interference of hemoglobin, bilirubin, and lipids on Roche Cobas 6000 assays," Clin. Chim. Acta 2011, 412, 1550-1553.

61. Haltmayer, M.; Mueller, T.; Gegenhuber, A.; Poelz, W. "Comparison of the automated AxSYM and ADVIA centaur immunoassays for homocysteine determination," Clin. Lab. 2004, 50, 175-180.

62. Hanson, N. Q.; Eckfeldt, J. H.; Schwichtenberg, K.; Aras, O.; Tsai, M. Y. "Interlaboratory variation of plasma total homocysteine measurements: Results of three successive homocysteine proficiency testing surveys," Clin. Chem. 2002, $48,1539-1545$.

63. de Silva, A. P.; Gunaratne, H. Q. N.; Gunnlaugsson, T.; Huxley, A. J. M.; McCoy, C. P.; Rademacher, J. T.; Rice, T. E. "Signaling recognition events with fluorescent sensors and switches," Chem. Rev. 1997, 97, 1515-1566.

64. Cho, D.-G.; Sessler, J. L. "Modern reaction-based indicator systems," Chem. Soc. Rev. 2009, 38, 1647-1662. 
65. Chen, X.; Zhou, Y.; Peng, X.; Yoon, J. "Fluorescent and colorimetric probes for detection of thiols," Chem. Soc. Rev. 2010, 39, 2120-2135.

66. Peng, H.; Chen, W.; Cheng, Y.; Hakuna, L.; Strongin, R.; Wang, B. "Thiol reactive probes and chemosensors," Sensors 2012, 12, 15907-15946.

67. Jung, H. S.; Chen, X. Q.; Kim, J. S.; Yoon, J. "Recent progress in luminescent and colorimetric chemosensors for detection of thiols," Chem. Soc. Rev. 2013, 42, 6019-6031.

68. Niu, L. Y.; Zheng, H. R.; Chen, Y. Z.; Wu, L. Z.; Tung, C. H.; Yang, Q. Z. "Fluorescent sensors for selective detection of thiols: expanding the intramolecular displacement based mechanism to new chromophores," Analyst 2014, 139, 1389-1395.

69. Goswami, S.; Manna, A.; Paul, S.; Das, A. K.; Nandi, P. K.; Maity, A. K.; Saha, P. "A turn on ESIPT probe for rapid and ratiometric fluorogenic detection of homocysteine and cysteine in water with live cell-imaging," Tetrahedron Lett. 2014, 55, 490-494.

70. Chen, H. L.; Zhao, Q.; Wu, Y. B.; Li, F. Y.; Yang, H.; Yi, T.; Huang, C. H. "Selective phosphorescence chemosensor for homocysteine based on an iridium(III) complex," Inorg. Chem. 2007, 46, 11075-11081.

71. Yu, C.; Zeng, F.; Luo, M.; Wu, S. "A silica nanoparticle-based sensor for selective fluorescent detection of homocysteine via interaction differences between thiols and particle-surface-bound polymers," Nanotechnology 2012, 23, 305503. 
72. Peng, H.; Wang, K.; Dai, C.; Williamson, S.; Wang, B. "Redox-based selective fluorometric detection of homocysteine," Chem. Commun. 2014, 50, 1366813671.

73. Dicaire, C.; Mess, J. N.; Furtado, M.; Bergeron, A.; Garofolo, F. "Impact of organic solvent additive on the integrity of plasma samples in bioanalysis by LCMS/MS," Bioanalysis 2013, 5, 2359-2370.

74. Wang, W. H.; Rusin, O.; Xu, X. Y.; Kim, K. K.; Escobedo, J. O.; Fakayode, S. O.; Fletcher, K. A.; Lowry, M.; Schowalter, C. M.; Lawrence, C. M.; Fronczek, F. R.; Warner, I. M.; Strongin, R. M. "Detection of homocysteine and cysteine," J. Am. Chem. Soc. 2005, 127, 15949-15958.

75. Barve, A.; Lowry, M.; Escobedo, J. O.; Huynh, K. T.; Hakuna, L.; Strongin, R. M. "Differences in heterocycle basicity distinguish homocysteine from cysteine using aldehyde-bearing fluorophores," Chem. Commun. 2014, 50, 8219-8222.

76. Abedinzadeh, Z. "Sulfur-centered reactive intermediates derived from the oxidation of sulfur compounds of biological interest," Can. J. Physiol. Pharmacol. 2001, 79, 166-170.

77. Akhlaq, M. S.; Schuchmann, H. P.; Vonsonntag, C. "The reverse of the repair reaction of thiols - H-abstraction at carbon by thiyl radicals," Int. J. Radiat. Biol. 1987, 51, 91-102.

78. Neta, P.; Fessende.Rw "Electron spin resonance study of radicals produced in irradiated aqueous solutions of thiols," J. Phys. Chem. 1971, 75, 2277-2283. 
79. Sjoberg, L.; Eriksen, T. E.; Revesz, L. "The reaction of the hydroxyl radical with glutathione in neutral and alkaline aqueous-solution," Radiat. Res. 1982, 89, 255263.

80. Zhao, R.; Lind, J.; Merenyi, G.; Eriksen, T. E. "Kinetics of one-electron oxidation of thiols and hydrogen abstraction by thiyl radicals from alpha-amino $\mathrm{C}-\mathrm{H}$ bonds," J. Am. Chem. Soc. 1994, 116, 12010-12015.

81. Wang, W. H.; Escobedo, J. O.; Lawrence, C. M.; Strongin, R. M. "Direct detection of homocysteine," J. Am. Chem. Soc. 2004, 126, 3400-3401.

82. Escobedo, J. O.; Wang, W.; Strongin, R. M. "Use of a commercially available reagent for the selective detection of homocysteine in plasma," Nat. Protocols 2006, 1, 2759-2762.

83. Hakuna, L.; Escobedo, J. O.; Lowry, M.; Barve, A.; McCallum, N.; Strongin, R. M. "A photochemical method for determining plasma homocysteine with limited sample processing," Chem. Commun. 2014, 50, 3071-3073.

84. Johnson Jr, C.; Gutowsky, H. "High-resolution ESR spectra of photochemically generated free radicals: The viologens," J. Chem. Phys. 1963, 39, 58.

85. Nauser, T.; Koppenol, W. H.; Schöneich, C. "Reversible hydrogen transfer reactions in thiyl radicals from cysteine and related molecules: Absolute kinetics and equilibrium constants determined by pulse radiolysis," J. Phys. Chem. B 2012, 116, 5329-5341. 
86. Wang, D.; Crowe, W. E.; Strongin, R. M.; Sibrian-Vazquez, M. "Exploring the $\mathrm{pH}$ dependence of viologen reduction by $\alpha$-carbon radicals derived from Hcy and Cys," Chem. Commun. 2009, 0, 1876-1878.

87. Bird, C. L.; Kuhn, A. T. "Electrochemistry of the viologens," Chem. Soc. Rev. 1981, $10,49-82$.

88. Cordobatorresi, S. I.; Gabrielli, C.; Hugotlegoff, A.; Torresi, R. "Electrochromic behavior of nickel-oxide electrodes .1. Identification of the colored state using quartz crystal microbalance," J. Electrochem. Soc. 1991, 138, 1548-1553.

89. Small, D.; Zaitsev, V.; Jung, Y. S.; Rosokha, S. V.; Head-Gordon, M.; Kochi, J. K. "Lntermolecular,pi-to-pi bonding between stacked aromatic dyads. Experimental and theoretical binding energies and near-IR optical transitions for phenalenyl radical/radical versus radical/cation dimerizations," J. Am. Chem. Soc. 2004, 126, 13850-13858.

90. Porter, W. W.; Vaid, T. P. "Isolation and characterization of phenyl viologen as a radical cation and neutral molecule," J. Org. Chem. 2005, 70, 5028-5035.

91. Lapkowski, M.; Bidan, G. "Electrochemical, Spectroelectrochemical and EPR Properties of Poly(Pyrrole-Viologens)," J. Electroanal. Chem. 1993, 362, 249256.

92. Coe, B. J.; Harries, J. L.; Helliwell, M.; Jones, L. A.; Asselberghs, I.; Clays, K.; Brunschwig, B. S.; Harris, J. A.; Garín, J.; Orduna, J. "Pentacyanoiron(II) as an electron donor group for nonlinear optics: Medium-responsive properties and 
comparisons with related pentaammineruthenium(II) complexes," J. Am. Chem. Soc. 2006, 128, 12192-12204.

93. Kamogawa, H.; Sato, S. "Redox photochromism of arylviologen crystals," Bull. Chem. Soc. Jpn. 1991, 64, 321-323.

94. Carballada, P. C.; Mourtzis, N.; Felici, M.; Bonnet, S.; Nolte, R. J. M.; Williams, R. M.; De Cola, L.; Feiters, M. C. "Variation of the viologen electron relay in cyclodextrin-based self-assembled systems for photoinduced hydrogen evolution from water," Eur. J. Org. Chem. 2012, 6729-6736.

95. Rautiola, D. M.S. Dissertation, Portland State University, 2012.

96. Lambert, M.; Rastede, E. E.; Decker, J.; Raymo, F. M. "Microwave-assisted synthesis of symmetric and asymmetric viologens," Tetrahedron Lett. 2010, 51, 5618-5620.

97. Uhlig, S.; Wendel, A. "The physiological consequences of glutathione variations," Life Sci. 1992, 51, 1083-1094.

98. Ballatori, N.; Krance, S. M.; Notenboom, S.; Shi, S. J.; Tieu, K.; Hammond, C. L. "Glutathione dysregulation and the etiology and progression of human diseases," Biol. Chem. 2009, 390, 191-214.

99. Health topics: Chronic diseases [Internet]. World Health Organization; c2013. Available from: http://www.who.int/topics/chronic_diseases/en.

100. Beutler, E.; Moroose, R.; Kramer, L.; Gelbart, T.; Forman, L. "Gammaglutamylcysteine synthetase deficiency and hemolytic-anemia," Blood 1990, 75, 271-273. 
101. Atkuri, K. R.; Cowan, T. M.; Kwan, T.; Ng, A.; Herzenberg, L. A.; Herzenberg, L. A.; Enns, G. M. "Inherited disorders affecting mitochondrial function are associated with glutathione deficiency and hypocitrullinemia," Proc. Natl. Acad. Sci. U. S. A. 2009, 106, 3941-3945.

102. Liu, H.; Wang, H.; Shenvi, S.; Hagen, T. M.; Liu, R.-M. "Glutathione metabolism during aging and in Alzheimer disease," Ann. N. Y. Acad. Sci. 2004, 1019, 346349.

103. Dauer, W.; Przedborski, S. "Parkinson's disease: Mechanisms and models," Neuron 2003, 39, 889-909.

104. Mancuso, M.; Orsucci, D.; LoGerfo, A.; Rocchi, A.; Petrozzi, L.; Nesti, C.; Galetta, F.; Santoro, G.; Murri, L.; Siciliano, G. "Oxidative stress biomarkers in mitochondrial myopathies, basally and after cysteine donor supplementation," $J$. Neurol. 2010, 257, 774-781.

105. Engel, J. M.; Muhling, J.; Kwapisz, M.; Heidt, M. "Glutamine administration in patients undergoing cardiac surgery and the influence on blood glutathione levels," Acta Anaesthesiol. Scand. 2009, 53, 1317-1323.

106. Joncourt, F.; Oberlischraummli, A. E.; Stadler, M.; Buser, K.; Franscini, L.; Fey, M. F.; Cerny, T. "Patterns of drug resistance parameters in adult leukemia," Leuk. Lymphoma 1995, 17, 101-109.

107. Perry, R. R.; Mazetta, J. A.; Levin, M.; Barranco, S. C. "Glutathione levels and variability in breast tumors and normal tissue," Cancer 1993, 72, 783-787. 
108. Cook, J. A.; Pass, H. I.; Iype, S. N.; Friedman, N.; DeGraff, W.; Russo, A.; Mitchell, J. B. "Cellular glutathione and thiol measurements from surgically resected human lung tumor and normal lung tissue," Cancer Res. 1991, 51, 42874294.

109. Balendiran, G. K.; Dabur, R.; Fraser, D. "The role of glutathione in cancer," Cell Biochem. Funct. 2004, 22, 343-352.

110. Monostori, P.; Wittmann, G.; Karg, E.; Turi, S. "Determination of glutathione and glutathione disulfide in biological samples: An in-depth review," J. Chromatogr. B 2009, 877, 3331-3346.

111. Raththagala, M.; Root, P. D.; Spence, D. M. "Dynamic monitoring of glutathione in erythrocytes, without a separation step, in the presence of an oxidant insult," Anal. Chem. 2006, 78, 8556-8560.

112. Rahman, I.; Kode, A.; Biswas, S. K. "Assay for quantitative determination of glutathione and glutathione disulfide levels using enzymatic recycling method," Nat. Protoc. 2006, 1, 3159-3165.

113. Kim, G.-J.; Lee, K.; Kwon, H.; Kim, H.-J. "Ratiometric fluorescence imaging of cellular glutathione," Org. Lett. 2011, 13, 2799-2801.

114. Lim, S.-Y.; Lee, S.; Park, S. B.; Kim, H.-J. "Highly selective fluorescence turn-on probe for glutathione," Tetrahedron Lett. 2011, 52, 3902-3904.

115. Lu, J. X.; Song, Y. C.; Shi, W.; Li, X. H.; Ma, H. M. "A long-wavelength fluorescent probe for imaging reduced glutathione in live cells," Sens. Actuators, $B$ 2012, 161, 615-620. 
116. Niu, L.-Y.; Guan, Y.-S.; Chen, Y.-Z.; Wu, L.-Z.; Tung, C.-H.; Yang, Q.-Z. "BODIPY-based ratiometric fluorescent sensor for highly selective detection of glutathione over cysteine and homocysteine," J. Am. Chem. Soc. 2012, 134, 18928-18931.

117. Matsumoto, T.; Urano, Y.; Shoda, T.; Kojima, H.; Nagano, T. "A thiol-reactive fluorescence probe based on donor-excited photoinduced electron transfer: Key role of ortho substitution," Org. Lett. 2007, 9, 3375-3377.

118. Guo, X. F.; Wang, H.; Guo, Y. H.; Zhang, H. S. "Selective spectrofluorimetric determination of glutathione in clinical and biological samples using 1,3,5,7tetramethyl-8-phenyl-(2-maleimide)-difluoroboradiaza-s-indacene," Anal. Chim. Acta 2009, 633, 71-75.

119. Shao, N.; Jin, J.; Wang, H.; Zheng, J.; Yang, R.; Chan, W.; Abliz, Z. "Design of bis-spiropyran ligands as dipolar molecule receptors and application to in vivo glutathione fluorescent probes," J. Am. Chem. Soc. 2010, 132, 725-736.

120. Liu, J.; Bao, C.; Zhong, X.; Zhao, C.; Zhu, L. "Highly selective detection of glutathione using a quantum-dot-based OFF-ON fluorescent probe," Chem. Commun. 2010, 46, 2971-2973.

121. Yin, J.; Kwon, Y.; Kim, D.; Lee, D.; Kim, G.; Hu, Y.; Ryu, J. H.; Yoon, J. "Cyanine-based fluorescent probe for highly selective detection of glutathione in cell cultures and live mouse tissues," J. Am. Chem. Soc. 2014, 136, 5351-5358.

122. Yang, X. F.; Huang, Q.; Zhong, Y. G.; Li, Z.; Li, H.; Lowry, M.; Escobedo, J. O.; Strongin, R. M. "A dual emission fluorescent probe enables simultaneous 
detection of glutathione and cysteine/homocysteine," Chem. Sci. 2014, 5, 21772183.

123. Guo, Y.; Yang, X.; Hakuna, L.; Barve, A.; Escobedo, J. O.; Lowry, M.; Strongin, R. M. "A fast response highly selective probe for the detection of glutathione in human blood plasma," Sensors 2012, 12, 5940-5950.

124. Strongin, R. M.; Guo, Y.; Hakuna, L.; Lowry, M. A.; Escobedo, J. O. Selective detection of thiols. Patent WO 2013/126816 A1, August 29, 2013.

125. Yang, X. F.; Guo, Y. X.; Strongin, R. M. "A seminaphthofluorescein-based fluorescent chemodosimeter for the highly selective detection of cysteine," Org. Biomol. Chem. 2012, 10, 2739-2741.

126. Wei, L.; Lucas, A.; Yue, J.; Lennox, R. B. "Enhanced cyclization rates of large rings induced by a micellar environment," Langmuir 1991, 7, 1336-1339.

127. Huang, X.; Dong, Z.; Liu, J.; Mao, S.; Xu, J.; Luo, G.; Shen, J. "Seleniummediated micellar catalyst: an efficient enzyme model for glutathione peroxidaselike catalysis," Langmuir 2007, 23, 1518-1522.

128. Stevens, R.; Stevens, L.; Price, N. C. "The stabilities of various thiol compounds used in protein purifications," Biochem. Educ. 1983, 11, 70-70.

129. Wu, G.; Fang, Y. Z.; Yang, S.; Lupton, J. R.; Turner, N. D. "Glutathione metabolism and its implications for health," J. Nutr. 2004, 134, 489-492.

130. Tang, B.; Liu, F.; Xu, K.; Tong, L. "A novel metallobridged bis(betacyclodextrin)s fluorescent probe for the determination of glutathione," FEBS J. 2008, 275, 1510-1517. 
131. Strongin, R. M.; Sibrian-Vazquez, M. Thiol detection. US Patent 20120276649 A1, Nov. 1, 2012.

132. Sibrian-Vazquez, M.; Escobedo, J. O.; Lowry, M.; Fronczek, F. R.; Strongin, R. M. "Field Effects Induce Bathochromic Shifts in Xanthene Dyes," J. Am. Chem. Soc. 2012, 134, 10502-10508.

133. Flaring, U. B.; Hebert, C.; Wernerman, J.; Hammarqvist, F.; Rooyackers, O. E. "Circulating and muscle glutathione turnover in human endotoxaemia," Clin. Sci. 2009, 117, 313-319.

134. Flaring, U. B.; Rooyackers, O. E.; Hebert, C.; Bratel, T.; Hammarqvist, F.; Wernerman, J. "Temporal changes in whole-blood and plasma glutathione in ICU patients with multiple organ failure," Intensive Care Med. 2005, 31, 1072-1078.

135. Moberly, J. B.; Logan, J.; Borum, P. R.; Story, K. O.; Webb, L. E.; Jassal, S. V.; Mupas, L.; Rodela, H.; Alghamdi, G. A.; Moran, J. E.; Wolfson, M.; Martis, L.; Oreopoulos, D. G. "Elevation of whole-blood glutathione in peritoneal dialysis patients by L-2-oxothiazolidine-4-carboxylate, a cysteine prodrug (Procysteine (R))," J. Am. Soc. Nephrol. 1998, 9, 1093-1099.

136. Michelet, F.; Gueguen, R.; Leroy, P.; Wellman, M.; Nicolas, A.; Siest, G. "Blood and plasma glutathione measured in healthy-subjects by HPLC - relation to sex, aging, biological variables, and life habits," Clin. Chem. 1995, 41, 1509-1517.

137. Orfanos, A. P.; Naylor, E. W.; Guthrie, R. "Ultramicromethod for estimation of total glutathione in dried blood spots on filter-paper," Anal. Biochem. 1980, 104, 70-74. 
138. HemogloBind ${ }^{\mathrm{TM}}$ hemoglobin depletion from hemolyzed serum/plasma [Internet]. Biotech Support $\quad$ Group; $\quad$ Available from: http://www.biotechsupportgroup.com/hemoglobind-hemoglobin-removal-andcapture.

139. Carru, C.; Zinellu, A.; Mario Pes, G.; Marongiu, G.; Tadolini, B.; Deiana, L. "Ultrarapid capillary electrophoresis method for the determination of reduced and oxidized glutathione in red blood cells," Electrophoresis 2002, 23, 1716-1721.

140. Piccoli, G.; Fiorani, M.; Biagiarelli, B.; Palma, F.; Potenza, L.; Amicucci, A.; Stocchi, V. "Simultaneous high-performance capillary electrophoretic determination of reduced and oxidized glutathione in red blood cells in the femtomole range," J. Chromatogr. A 1994, 676, 239-246. 


\section{APPENDIX A: SUPPORTING INFORMATION}

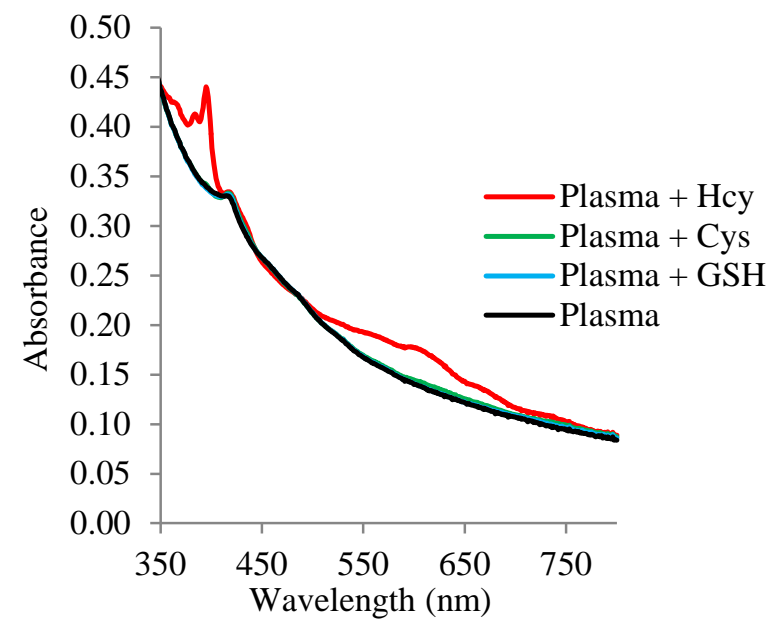

Figure A1. Spectral response of $\mathrm{MV}^{2+}$ towards various spiked thiols in human blood plasma upon irradiation. Absorption spectra of solutions of $\mathrm{MV}^{2+}(50 \mathrm{mM})$ in human blood plasma and $0.5 \mathrm{M}$ Tris buffer (pH 7.0) spiked with $1.5 \mu \mathrm{M}$ Hcy, $25 \mu \mathrm{M}$ Cys and $0.6 \mu \mathrm{M}$ GSH. Plasma (10\% v/v) was added to an argonsaturated solution of viologen, thiol and buffer and irradiated for 15 min using a Reptisun ${ }^{\mathrm{TM}}$ lamp.

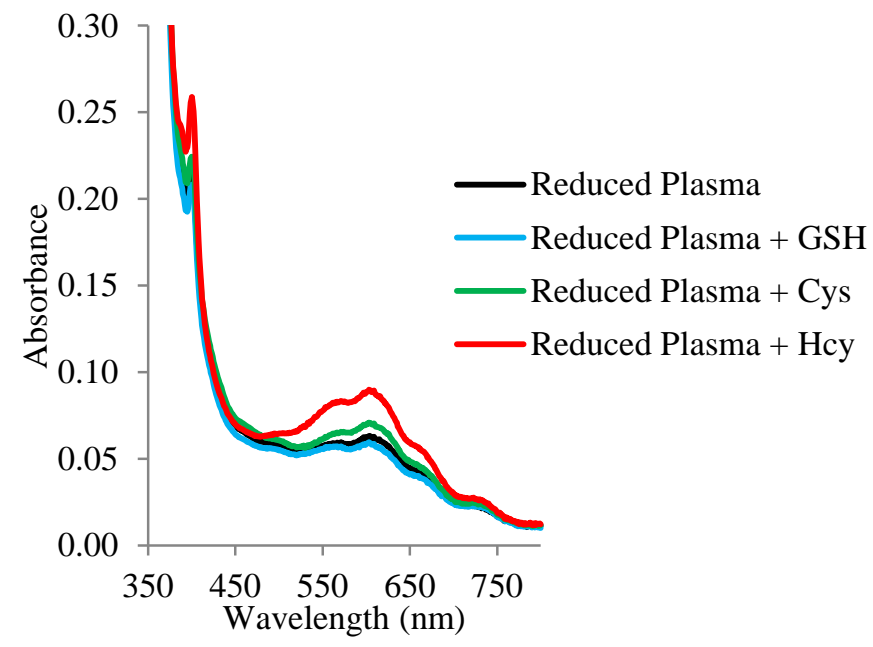

Figure A2. Spectral response of $\mathrm{BV}^{2+}$ towards various spiked thiols in reduced human blood plasma upon irradiation. Absorption spectra of solutions of $\mathrm{BV}^{2+}(20 \mathrm{mM})$ in reduced human blood plasma and $0.5 \mathrm{M}$ Tris buffer ( $\mathrm{pH}$ 7.0) spiked with $1.5 \mu \mathrm{M}$ Hcy, $25 \mu \mathrm{M}$ Cys and $0.6 \mu \mathrm{M}$ GSH. Plasma was incubated for $1 \mathrm{~h}$ with TCEP Gel and centrifuged at $1000 \mathrm{rpm}$ for $5 \mathrm{~min}$. The reduced, filtered plasma (10\% v/v) was added to an argon-saturated solution of viologen, thiol and buffer and irradiated for 15 min using a Reptisun ${ }^{\mathrm{TM}}$ lamp. 




Figure A3. Spectral response of $\mathrm{BV}^{2+}$ towards various spiked thiols in reduced human plasma upon irradiation. Absorption spectra of solutions of $\mathrm{BV}^{2+}(20 \mathrm{mM})$ in reduced human blood plasma and $0.5 \mathrm{M}$ Tris buffer ( $\mathrm{pH}$ 7.0) spiked with increasing concentrations of thiols. Plasma was incubated for $1 \mathrm{~h}$ with TCEP Gel followed by centrifugation at $1000 \mathrm{rpm}$ for $5 \mathrm{~min}$. The reduced plasma (10\% v/v) was added to an argon-saturated solution of viologen, thiol and buffer and irradiated for 15 min using a Reptisun ${ }^{\mathrm{TM}}$ lamp.

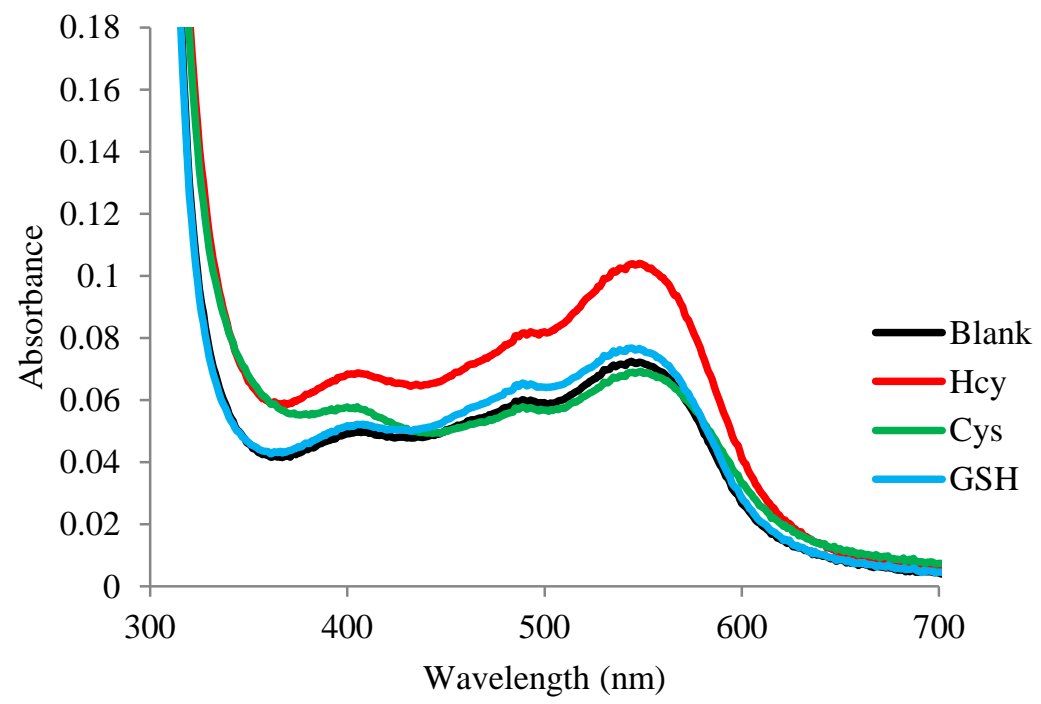

Figure A.4 Spectral response of $\mathrm{ACNV}^{2+}(\mathbf{2 6})$ towards various amino thiols at $\mathrm{pH}$ 8. Absorption spectra of solutions of $\mathrm{ACNV}^{2+}(350 \mu \mathrm{M})$ in thiol solutions $(15 \mu \mathrm{M} \mathrm{Hcy,} 250 \mu \mathrm{M}$ Cys and $6 \mu \mathrm{M} \mathrm{GSH})$ buffered at $\mathrm{pH}$ 8 (Phosphate buffer $(80 \mathrm{mM})$ at $10 \mathrm{~min}$. 


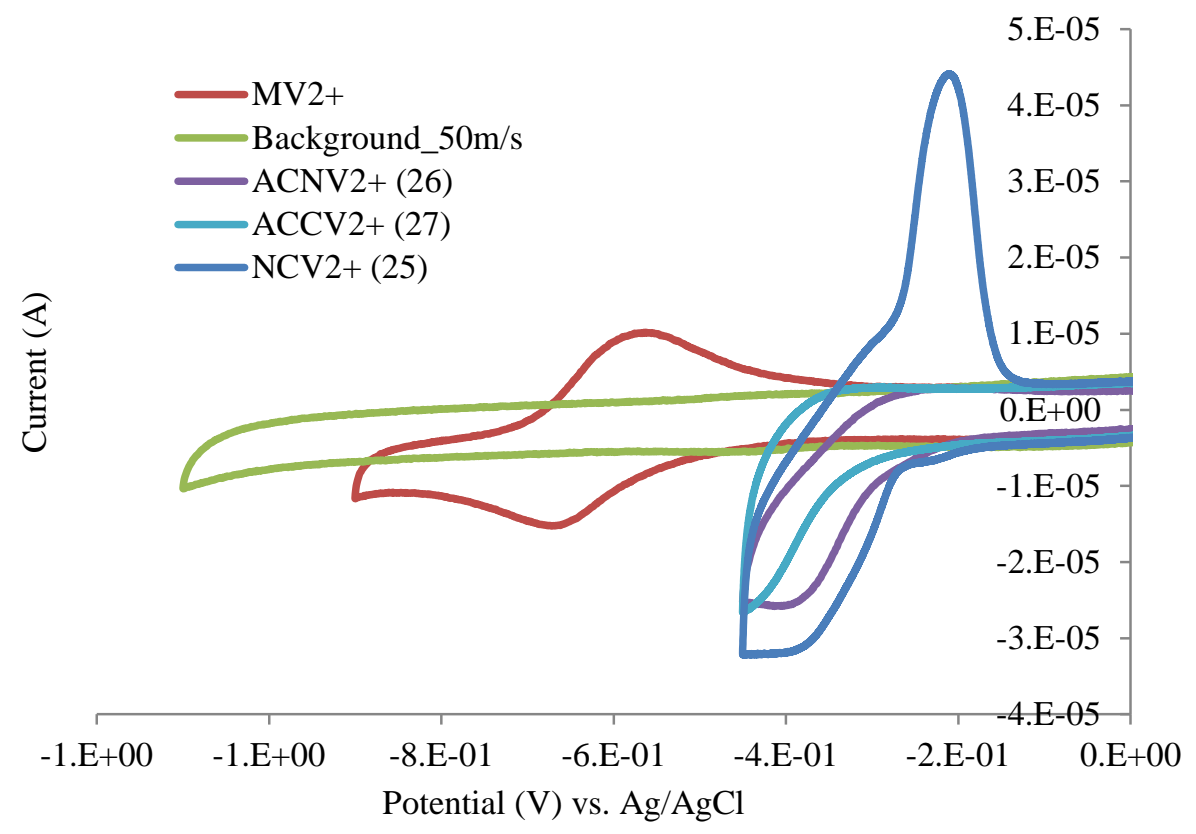

Figure A.5 Cyclic voltammograms of current $v s$ potential of asymmetric viologens and methyl viologen (4 $\mathrm{mM}$ ) in phosphate buffer at $50 \mathrm{mV} / \mathrm{s}$.



Figure A.6 Cyclic voltammograms of current $v s$ potential of $\mathbf{2 4}$ and $25(4 \mathrm{mM})$ in phosphate buffer at 100 $\mathrm{mV} / \mathrm{s}$. 


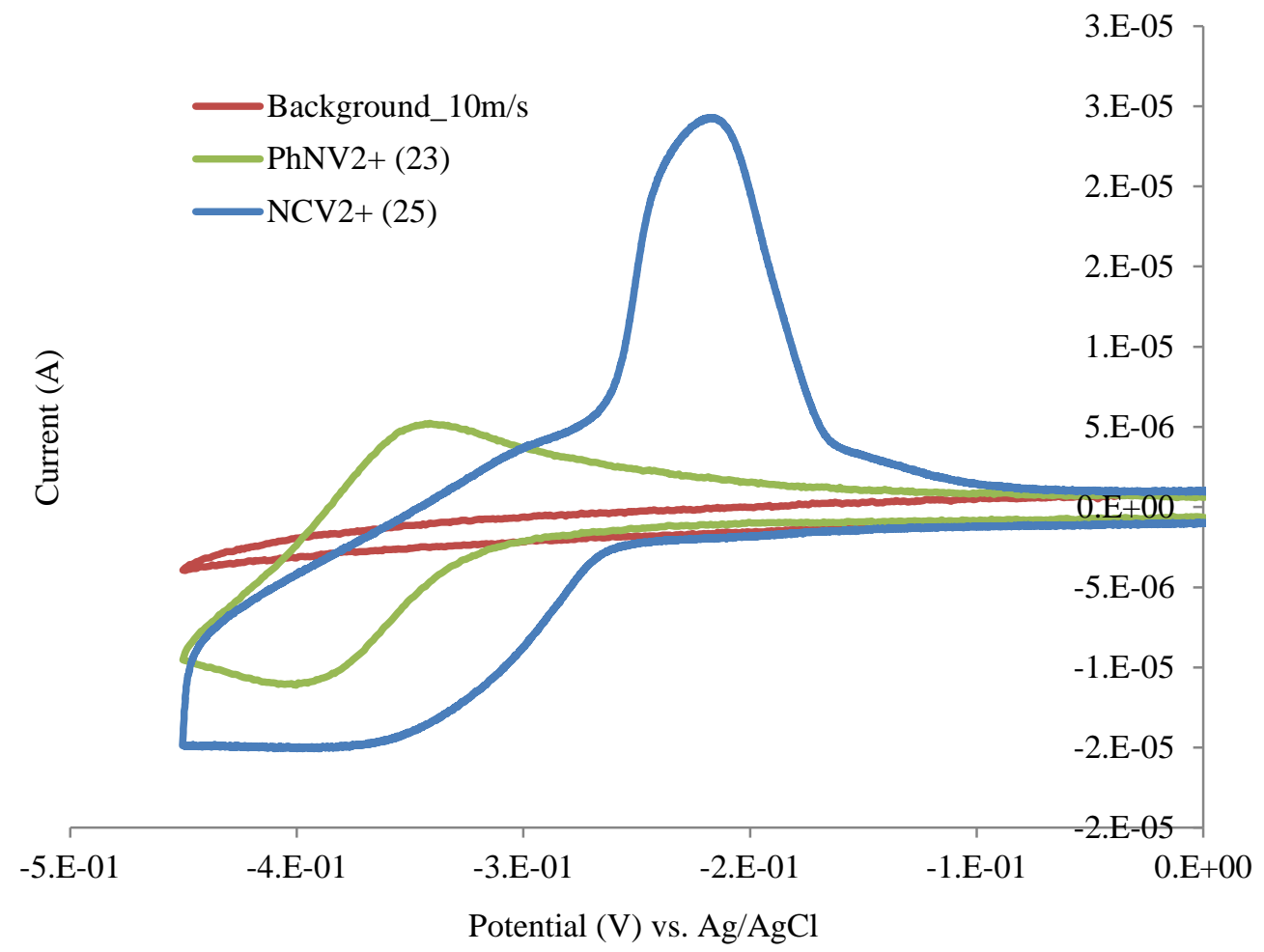

Figure A.7 Cyclic voltammograms of current $v s$ potential of 23 and $25(4 \mathrm{mM})$ in phosphate buffer at 10 $\mathrm{mV} / \mathrm{s}$. 


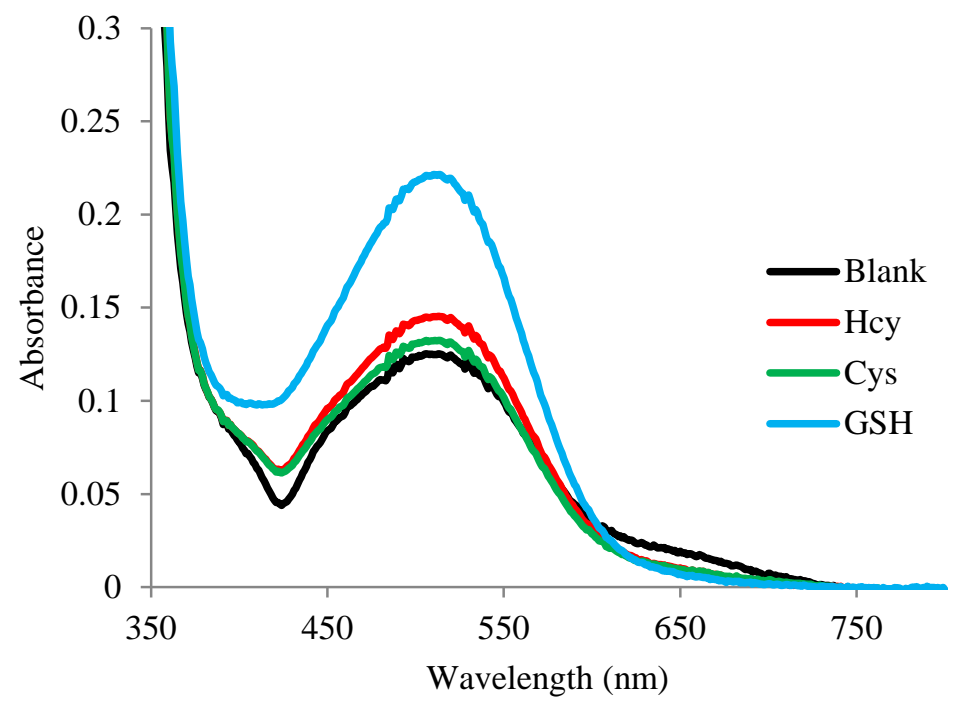

Figure A.8 Spectral response of $\mathrm{PhCV}^{2+}(\mathbf{2 4})$ towards various amino thiols at pH 9. Absorption spectra of solutions of $\mathrm{PhCV}^{2+}(500 \mu \mathrm{M})$ in thiol solutions $(500 \mu \mathrm{M})$ buffered at $\mathrm{pH} 9$ (Tris buffer $(200 \mathrm{mM})$.

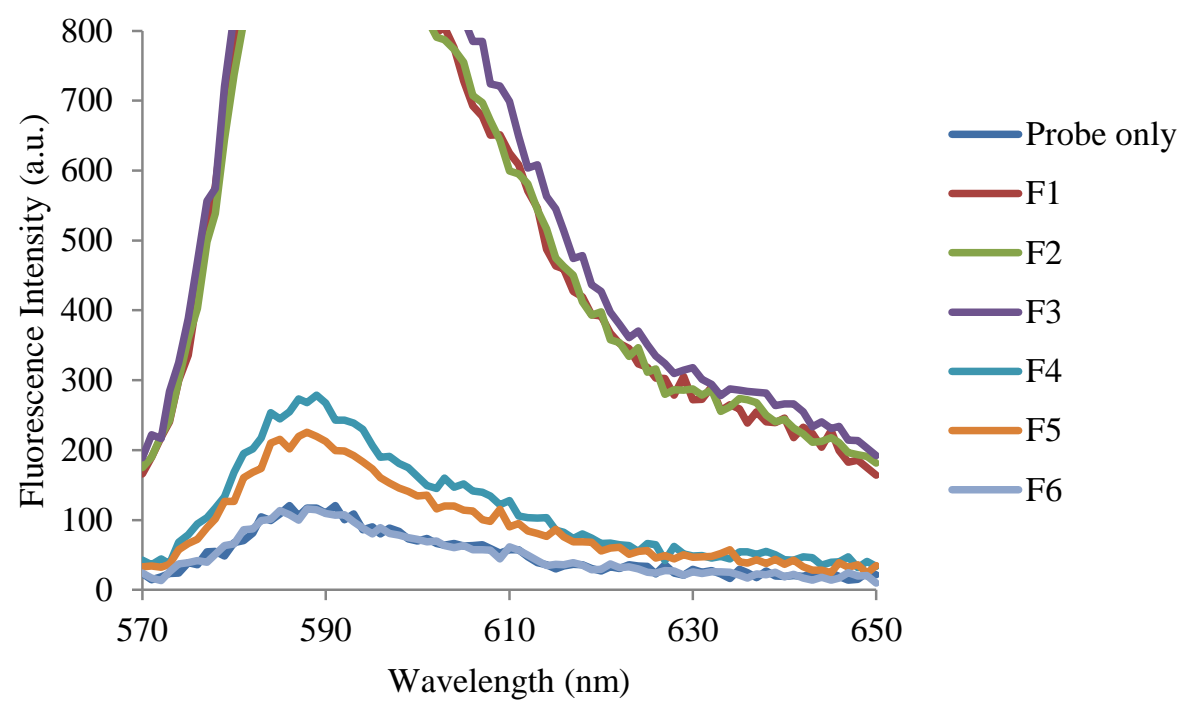

Figure A9 Spectral response of $\mathbf{5 0}$ towards various commercial GSH fractions at $\mathrm{pH}$ 7.4. Fluorescence spectra $(\lambda \mathrm{ex}=565 \mathrm{~nm})$ of $\mathbf{5 0}(2.5 \mu \mathrm{M})$ in solutions of GSH fractions and $2.0 \mathrm{mM}$ CTAB media buffered at pH 7.4 (phosphate buffer, $50 \mathrm{mM}$ ). Spectra were taken immediately upon addition of the probe. 


\section{APPENDIX B: CHARACTERIZATION OF COMPOUNDS}

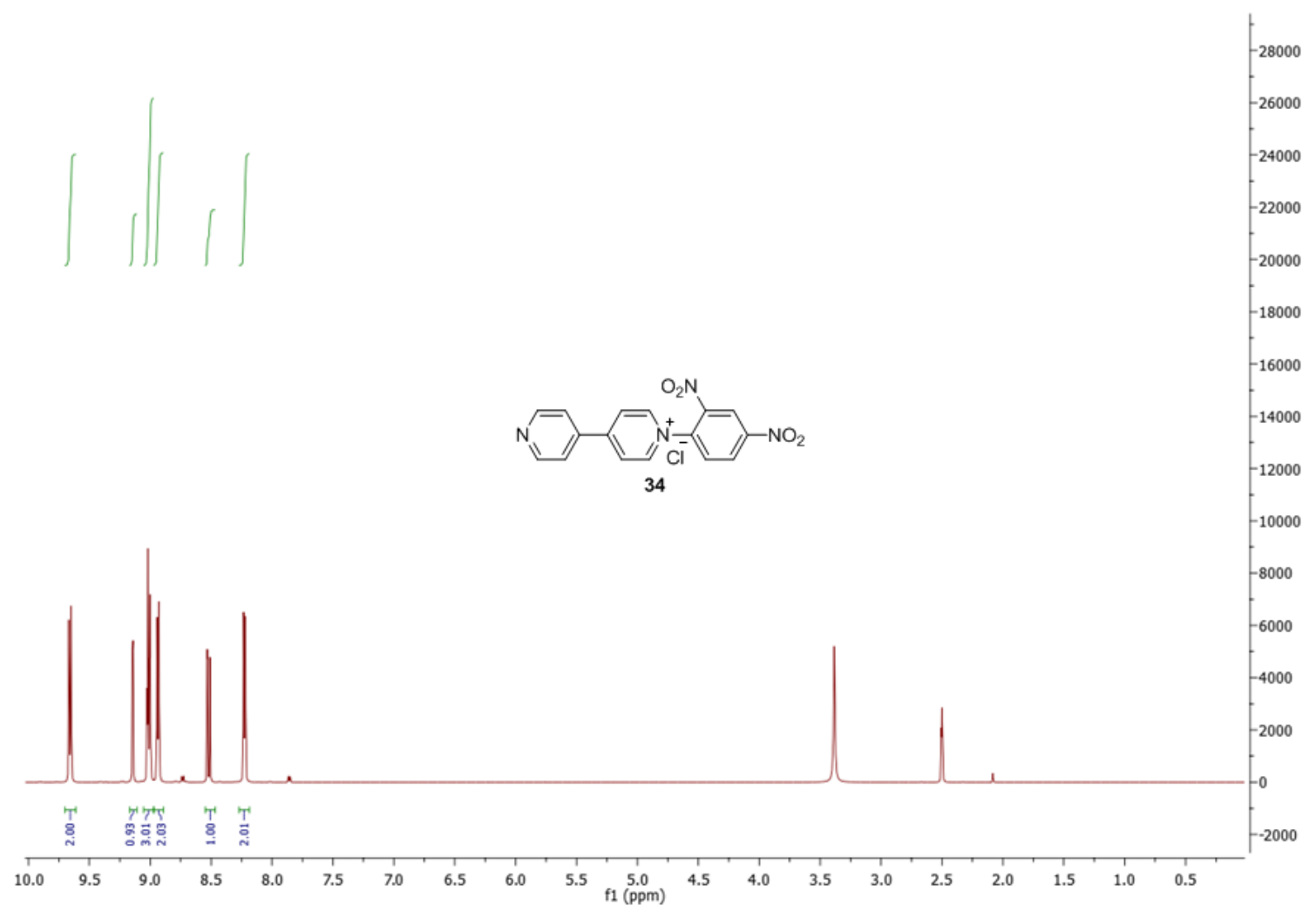




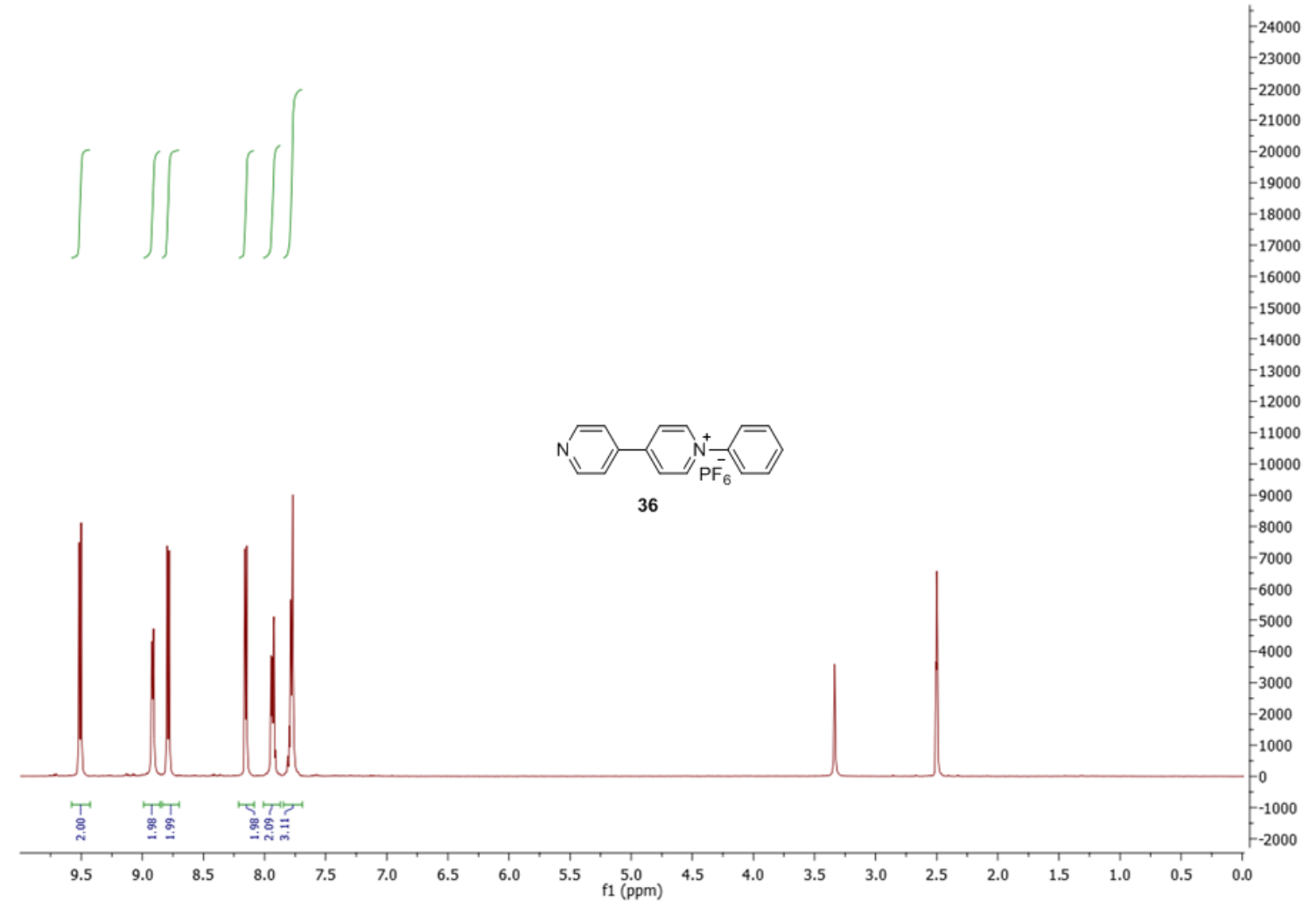









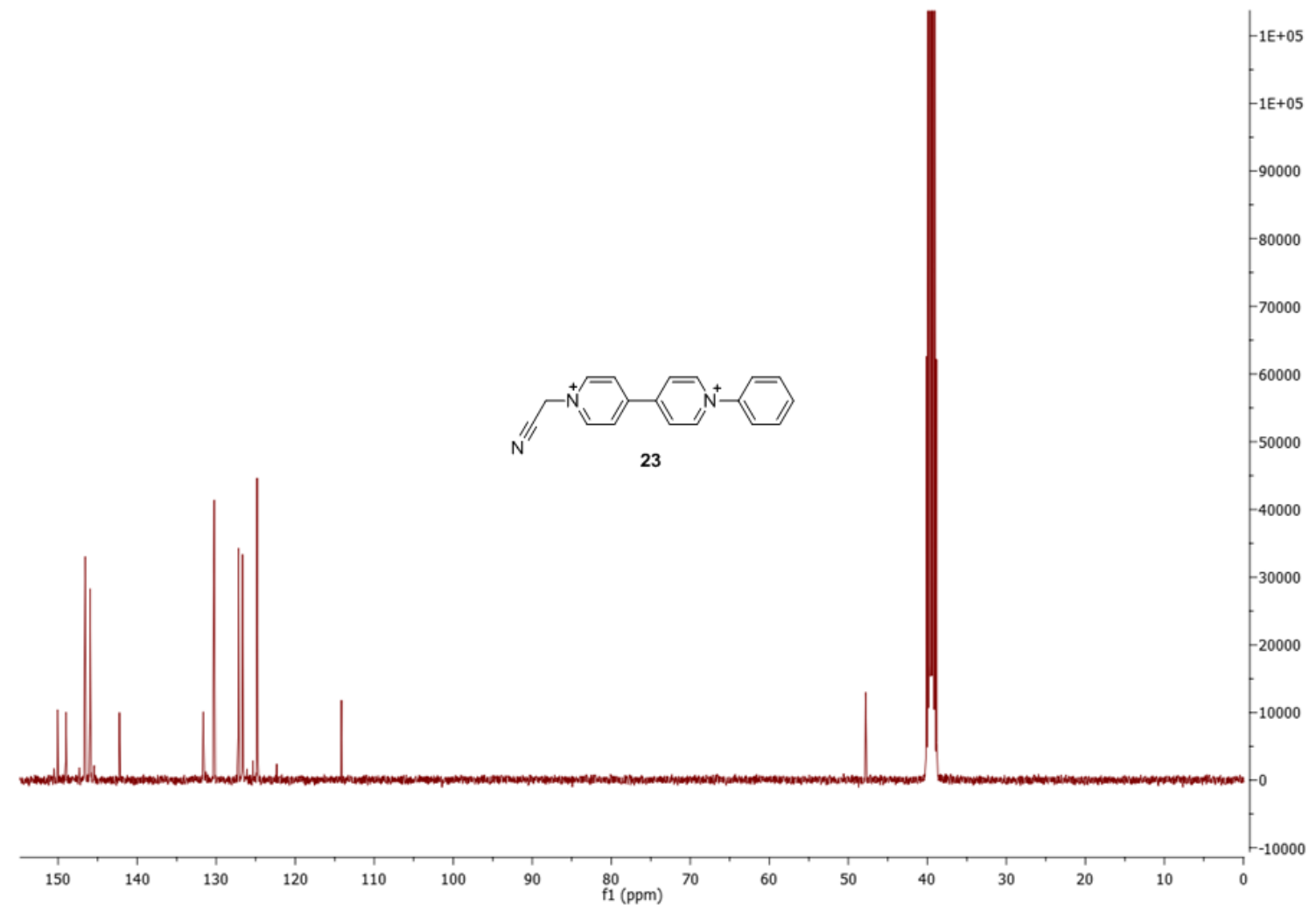




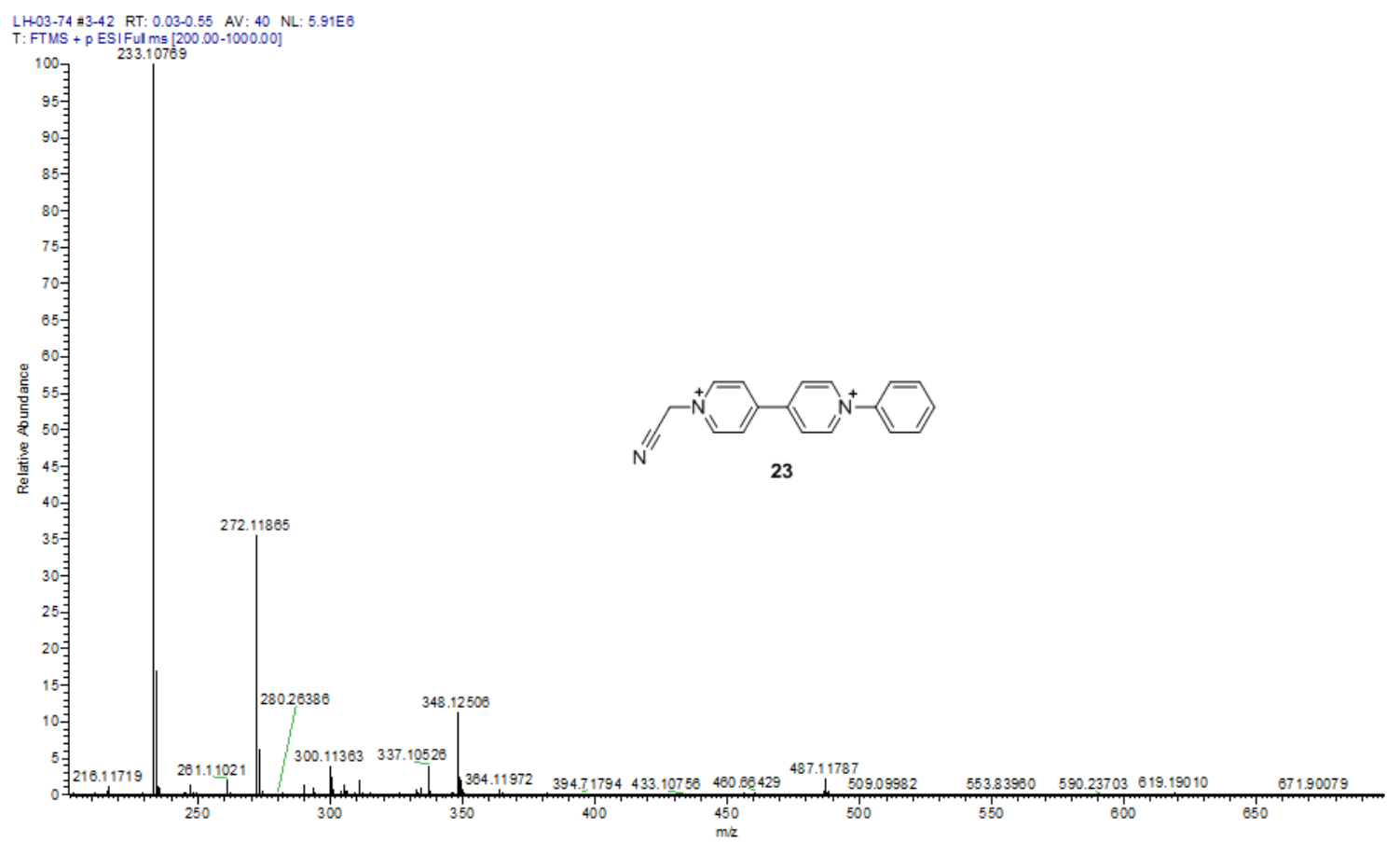









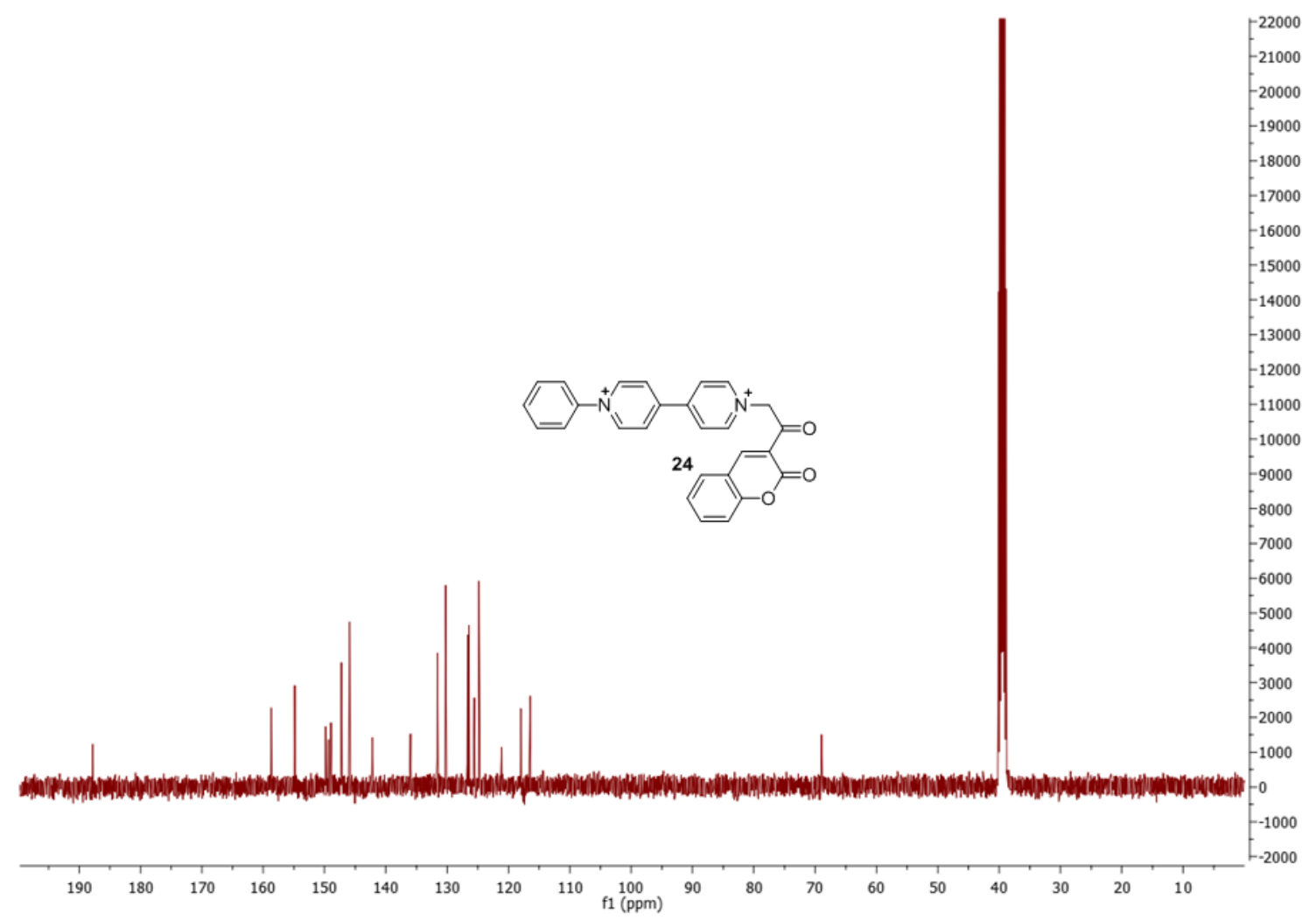


LH-03-8E $\# 2-50$ RT: $0.02-0.68$ AV: 49 NL: 2.14 E6

T: FTMS + p ESI Full ms [200.00-1000.00], 247.122 ,


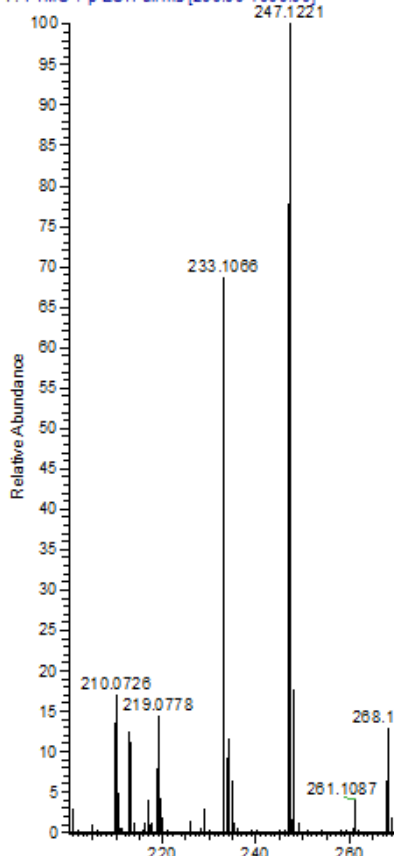


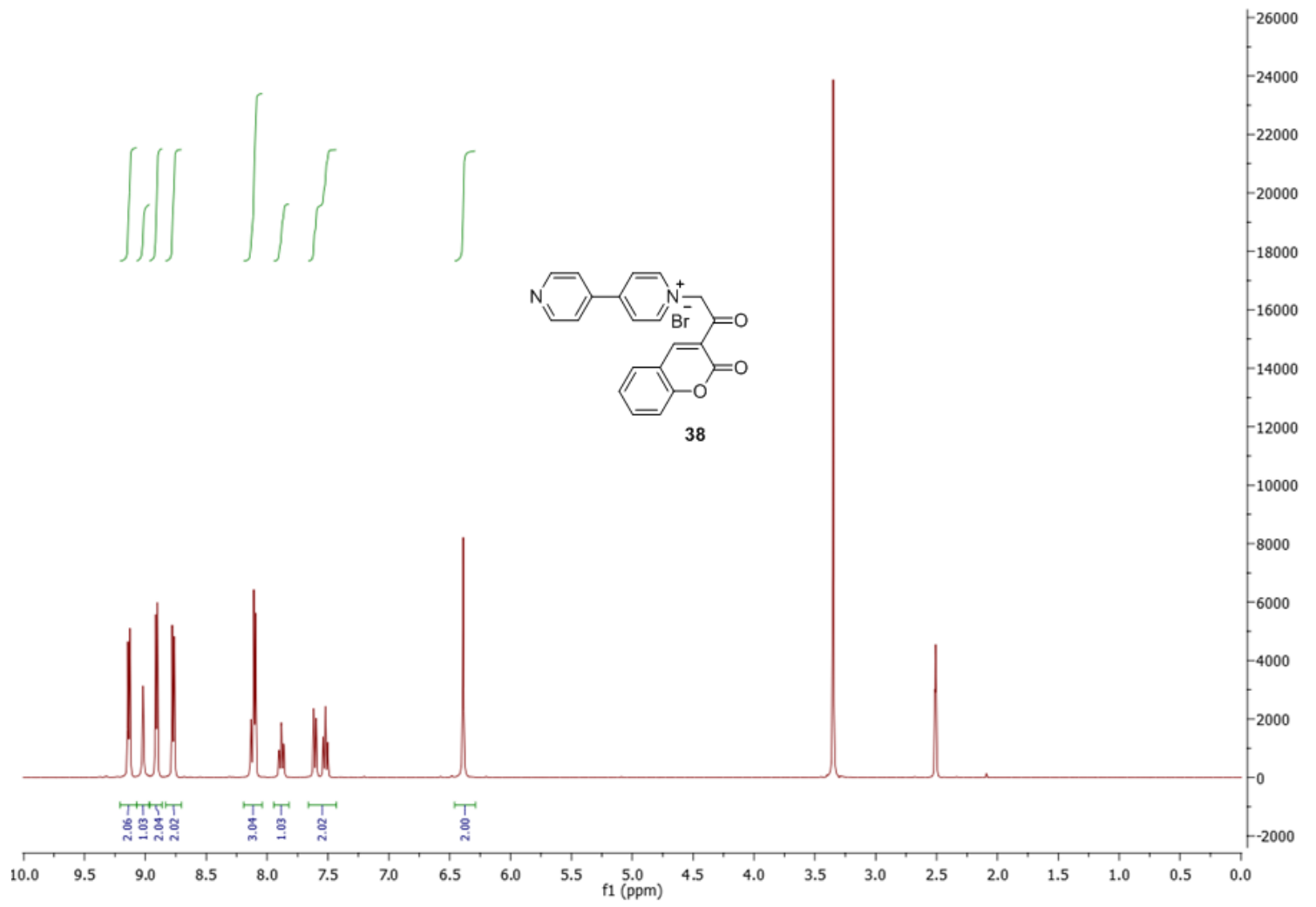








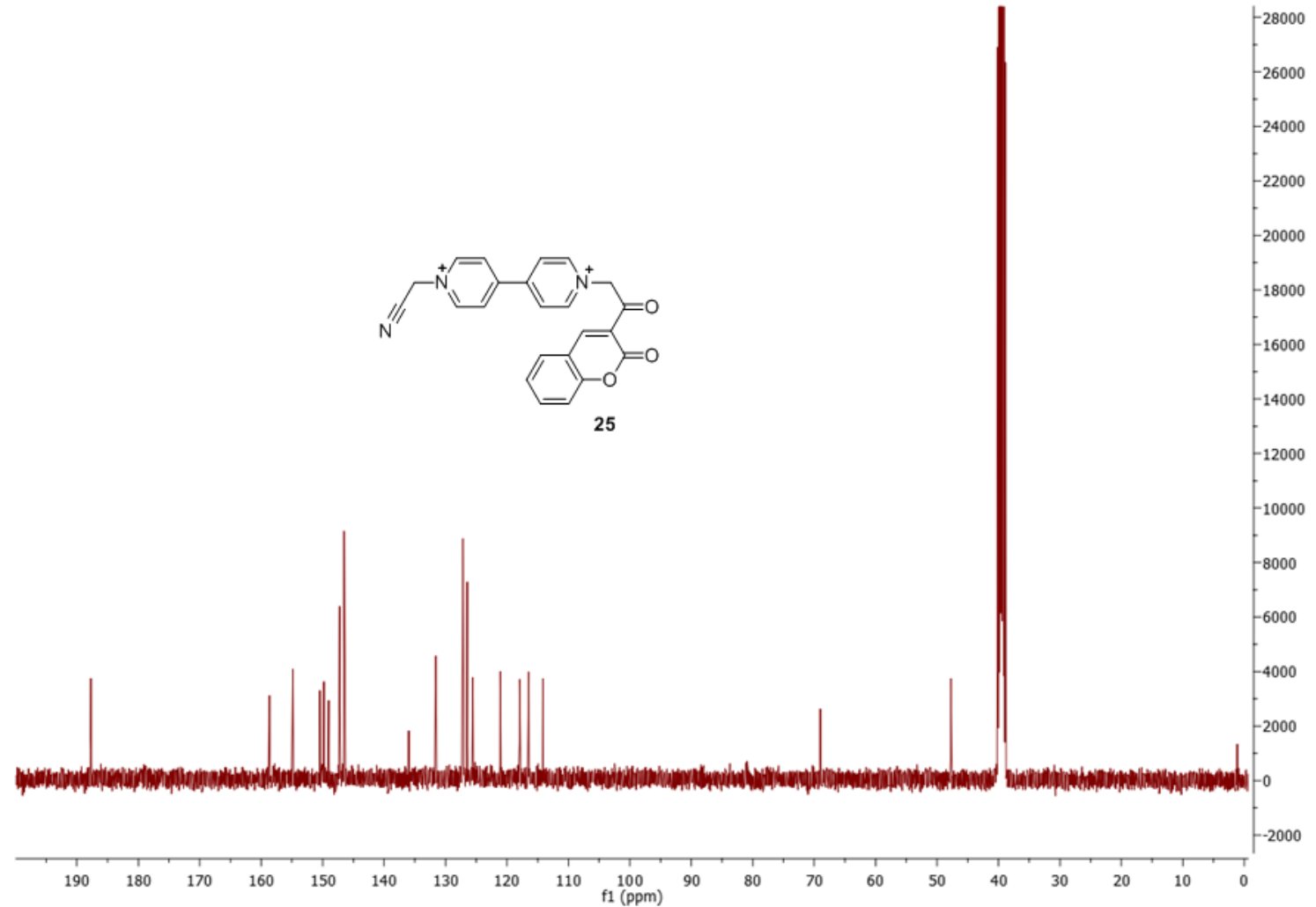

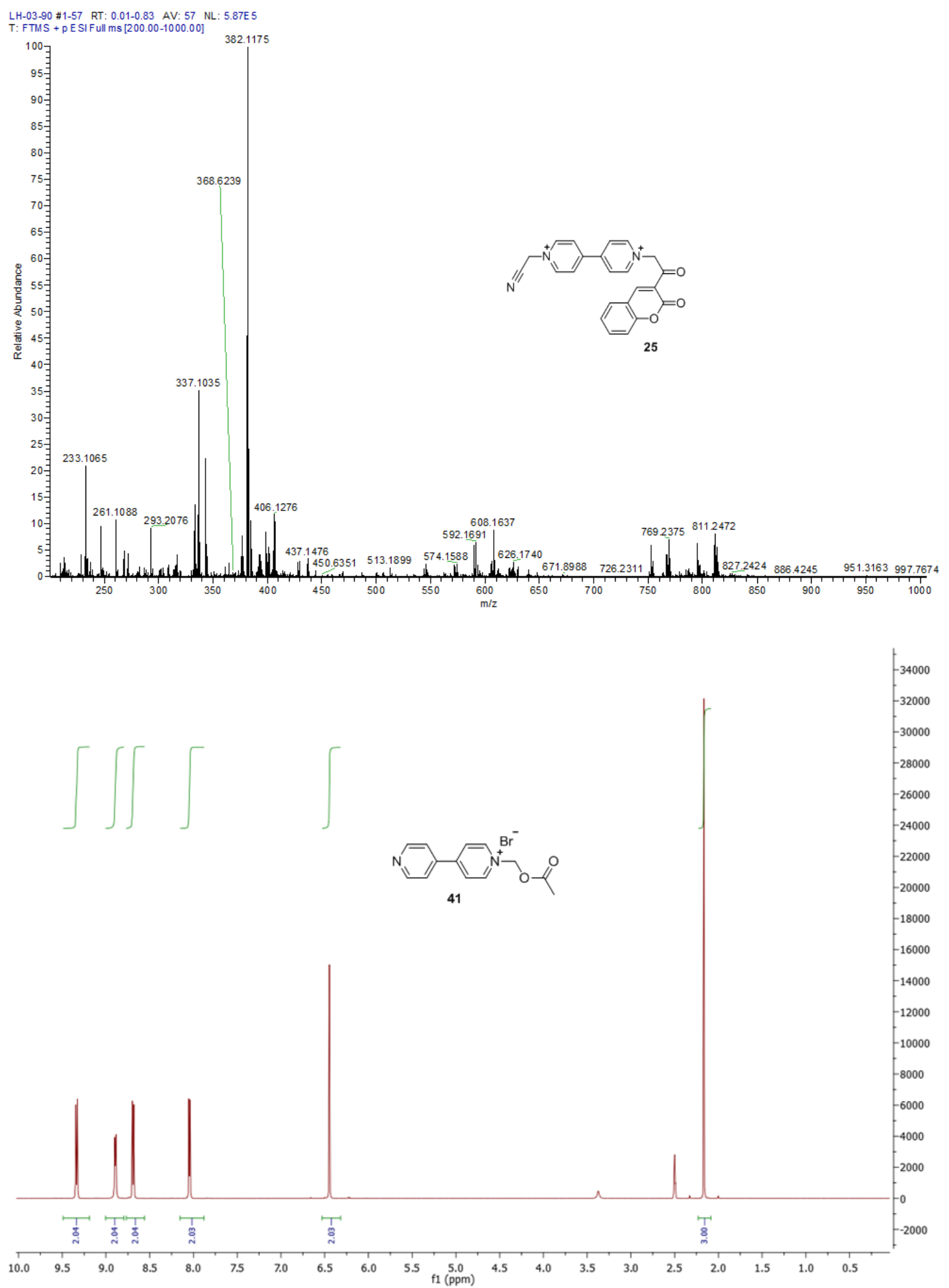




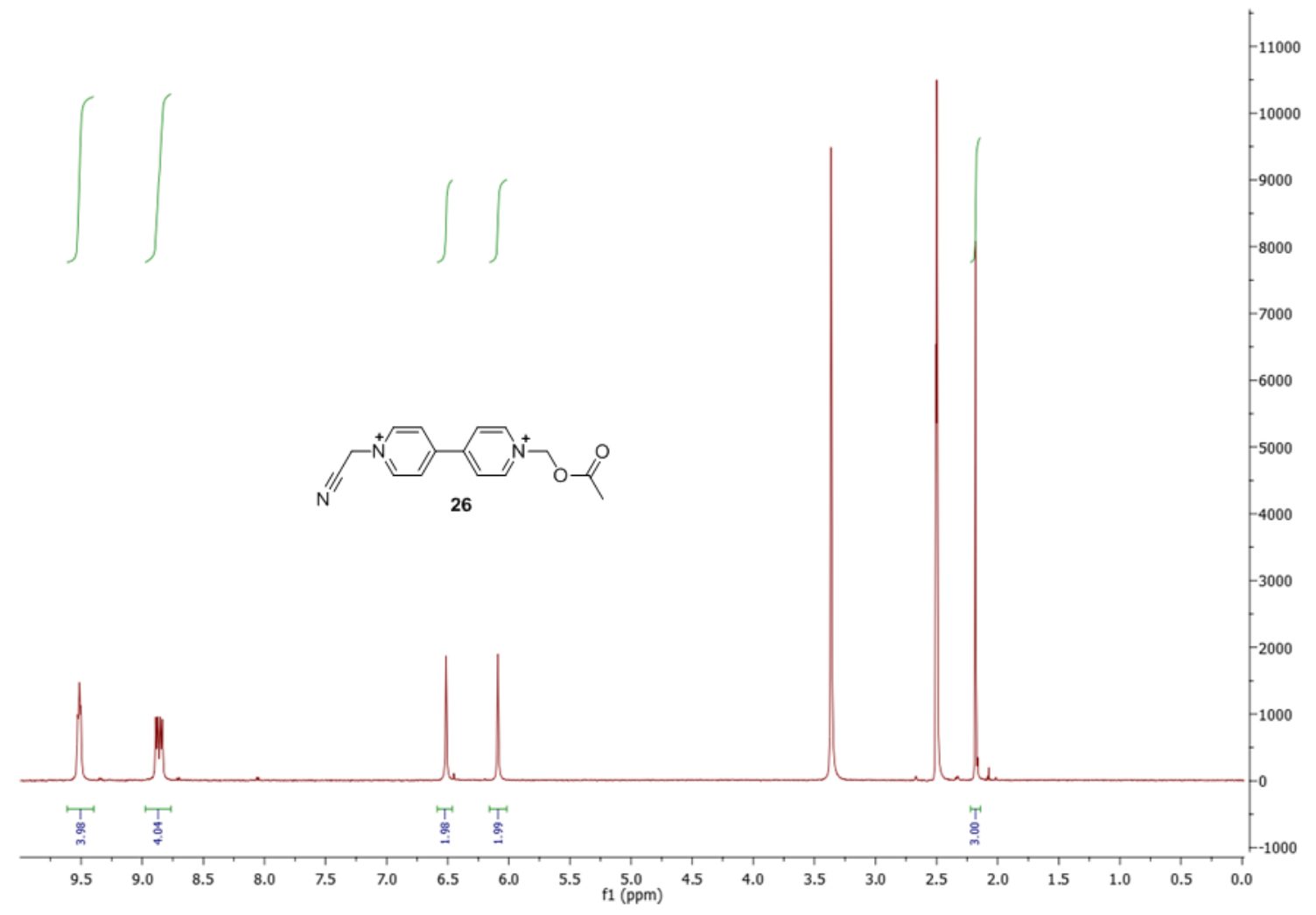


Oc08-2014

C13CPD DMSO \{C:|Bruker|TOPSPIN\} Strongin-Lovemore 3

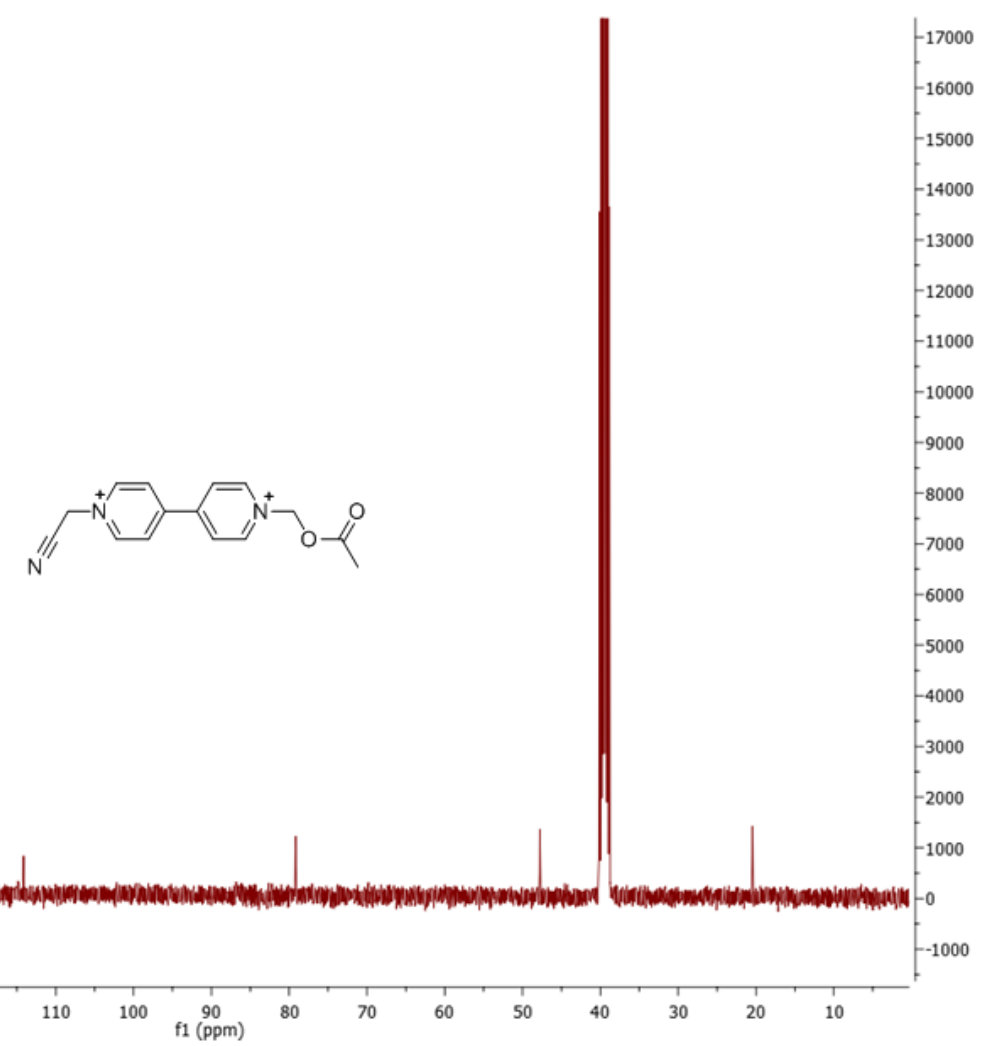









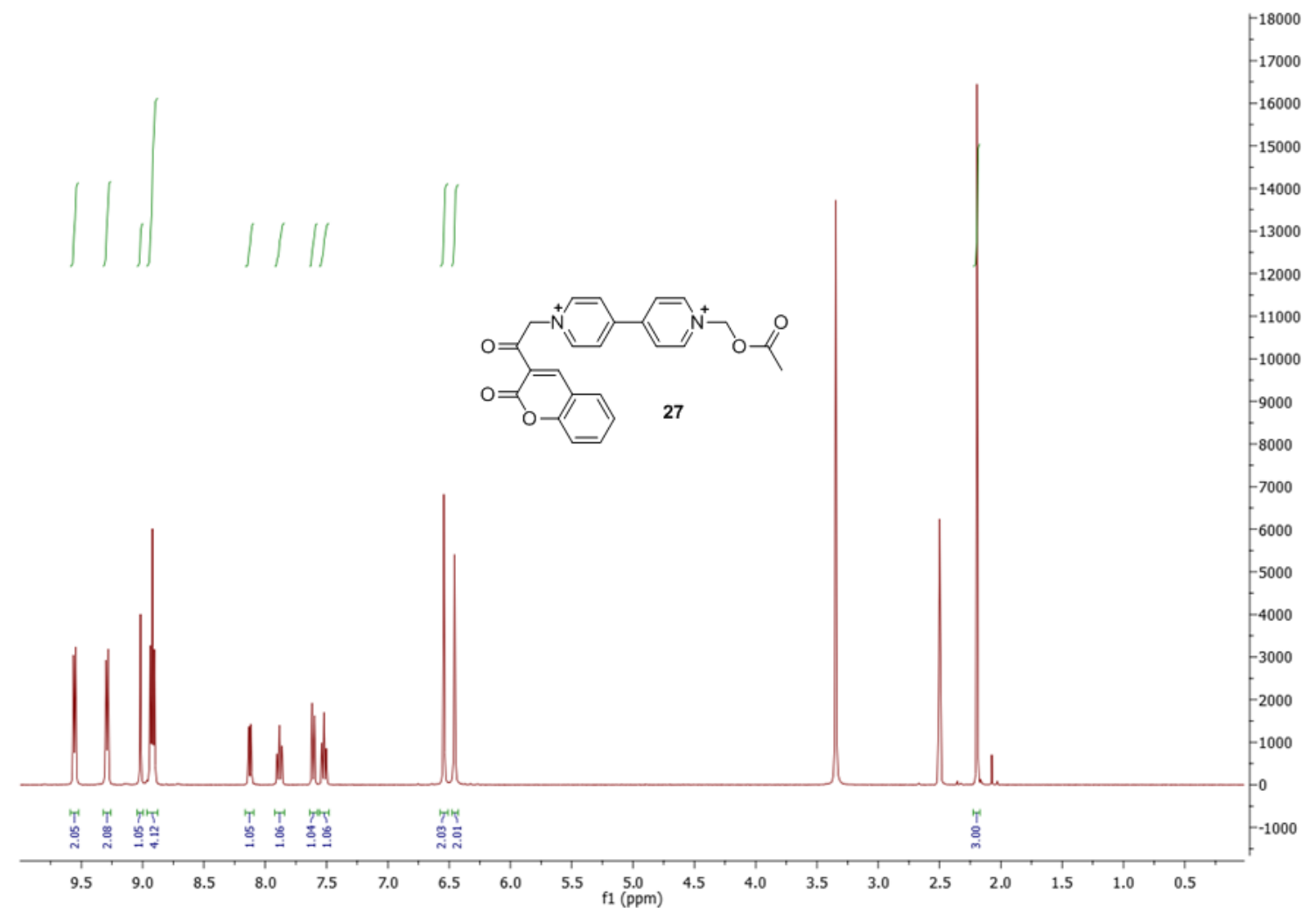









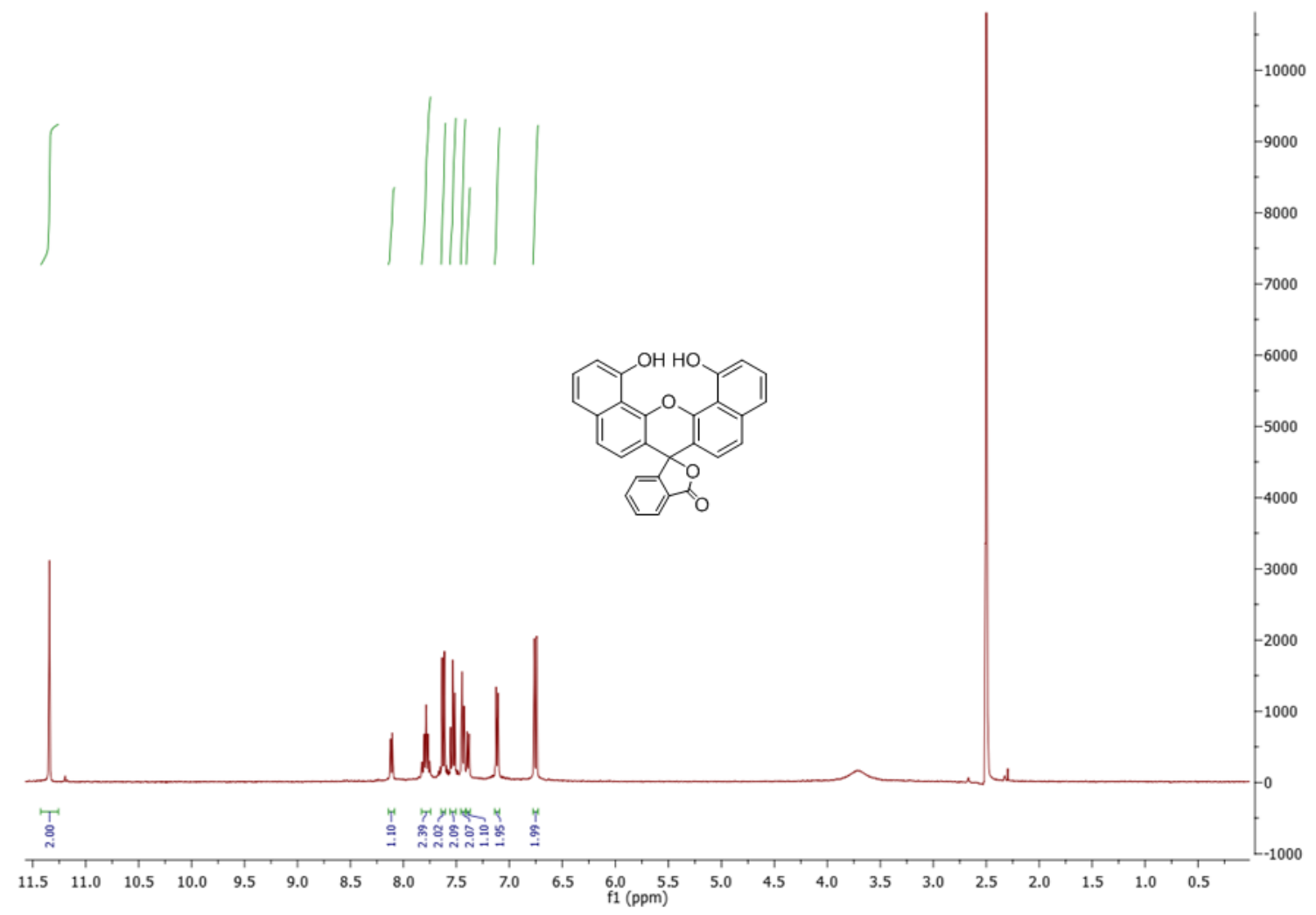




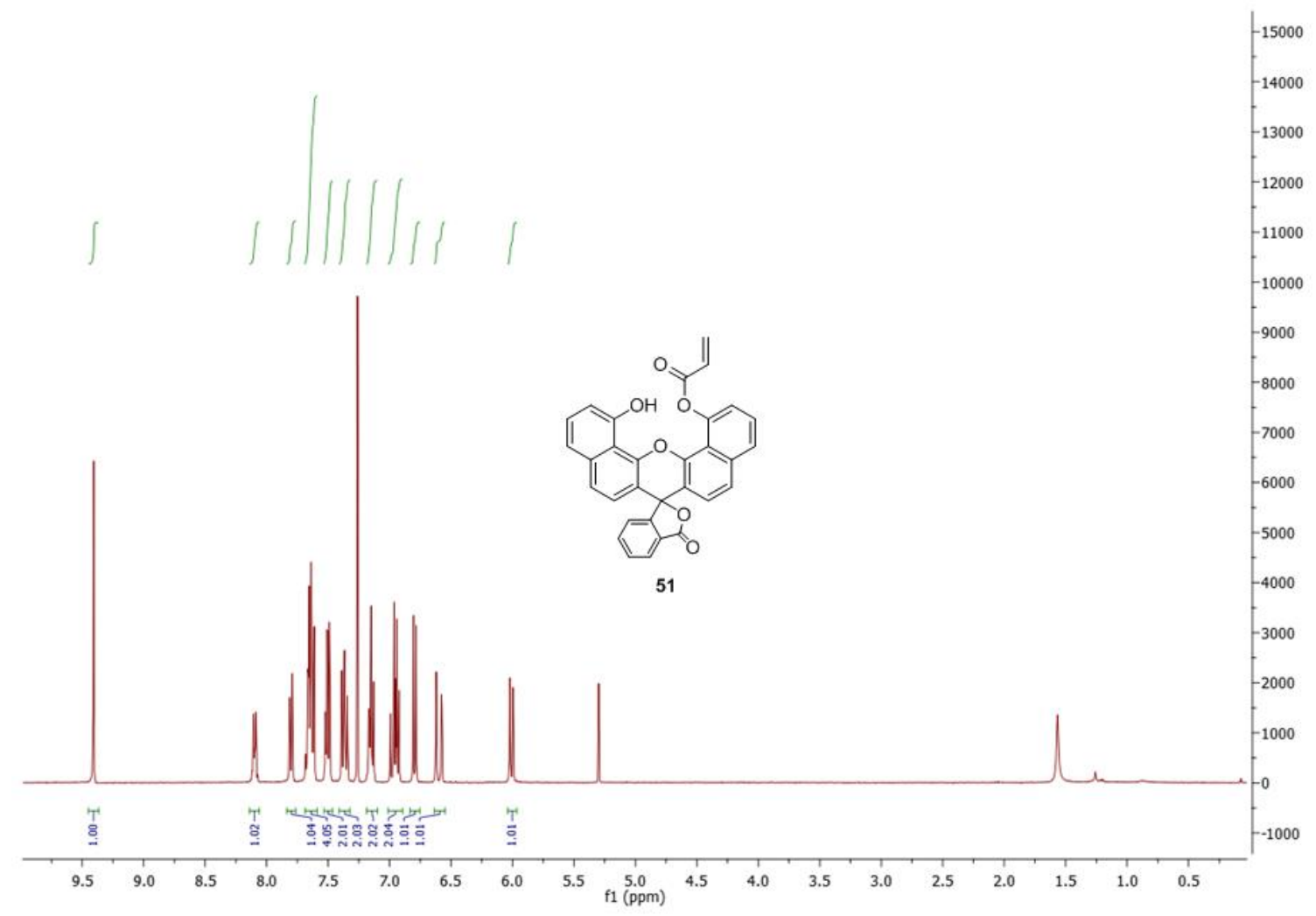



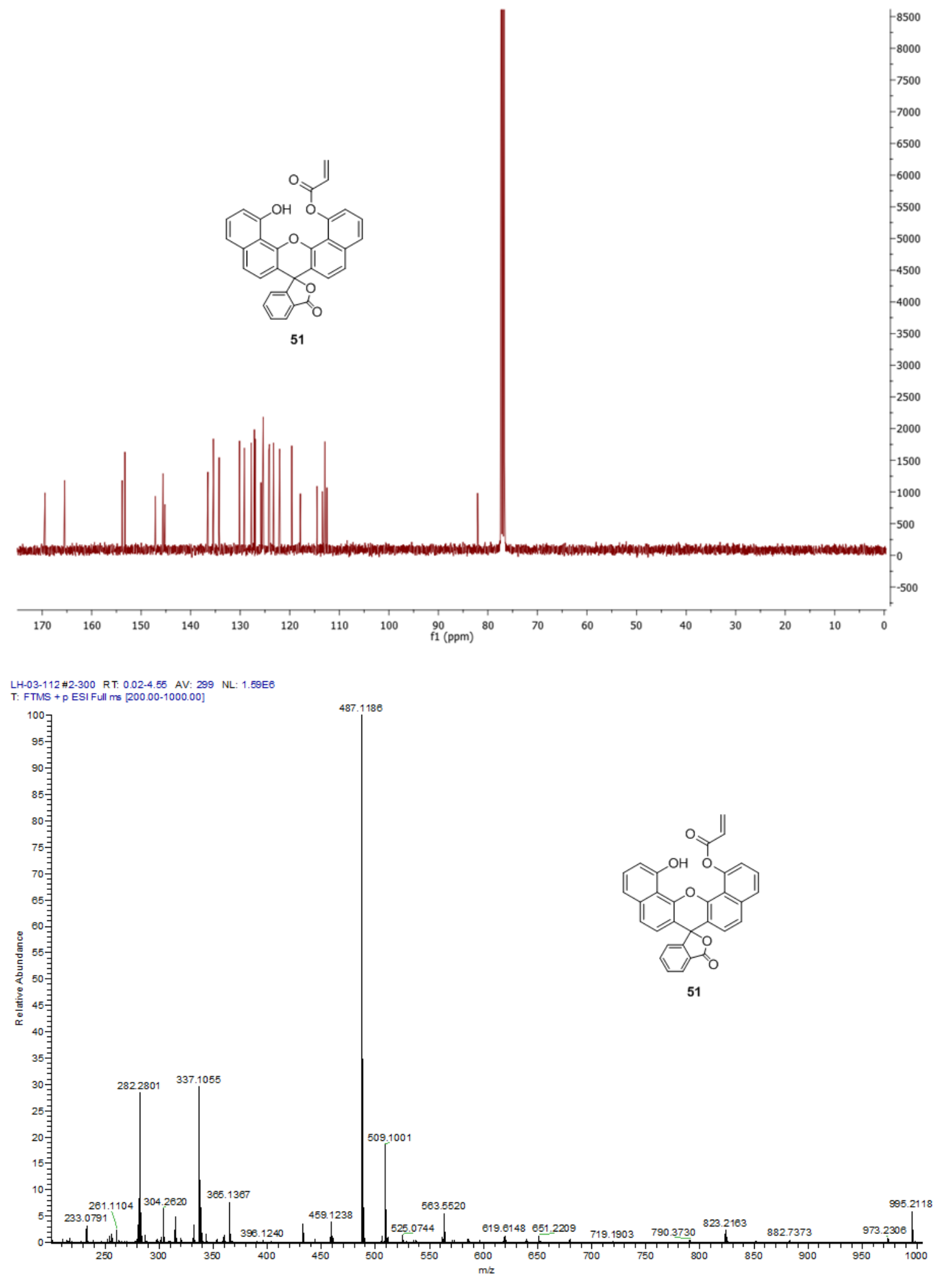